University of Tennessee Health Science Center

UTHSC Digital Commons

\title{
The Roles of Nuclear Receptor NR4A1 in Cancer Cell Proliferation and Skeletal Muscle Differentiation
}

\author{
Alexa Farmer \\ University of Tennessee Health Science Center
}

Follow this and additional works at: https://dc.uthsc.edu/dissertations

Part of the Medical Cell Biology Commons, and the Neoplasms Commons

\section{Recommended Citation}

Farmer, Alexa (http://orcid.org/0000-0002-8213-5253), "The Roles of Nuclear Receptor NR4A1 in Cancer Cell Proliferation and Skeletal Muscle Differentiation" (2016). Theses and Dissertations (ETD). Paper 403. http://dx.doi.org/10.21007/etd.cghs.2016.0411.

This Dissertation is brought to you for free and open access by the College of Graduate Health Sciences at UTHSC Digital Commons. It has been accepted for inclusion in Theses and Dissertations (ETD) by an authorized administrator of UTHSC Digital Commons. For more information, please contact jwelch30@uthsc.edu. 


\title{
The Roles of Nuclear Receptor NR4A1 in Cancer Cell Proliferation and Skeletal Muscle Differentiation
}

\begin{abstract}
Nuclear receptors (NRs) constitute a major class of drug targets in the treatment of various cancer types. NRs respond to cellular signals and become activated upon ligand binding to transcriptionally modulate expression of target genes. NR4A1 (Nur77) is a member of the NR4A family of nuclear receptors and displays an oncogenic profile in many cancer models. It is often upregulated in adult solid malignancies and is known to promote cell proliferation and survival. Knockdown studies of NR4A1 in cancer cell lines results in decreased cell growth and angiogenesis and increased apoptosis, suggesting NR4A1 is an oncogenic protein. Due to the elevated levels of NR4A1 in cancer, it is important to determine the regulatory mechanisms behind this expression pattern.
\end{abstract}

One such mechanism is through microRNAs (miRNAs), which regulate gene expression by binding to the $3^{\prime} U T R$ of target mRNA and effectively inhibit translation into protein. Prior to

this study, no miRNAs had been identified to directly target NR4A1. By using luciferase reporter assays, we identified miR-124, miR-15a, and miR-224 as potential NR4A1 regulators. The direct binding of these miRNAs to their potential seed regions within the 3'UTR of NR4A1 was confirmed by mutagenesis of their respective seed sequences. This abrogated the binding and thereby confirmed the direct targeting of these miRNAs to these particular sequences. To further study the relationship between NR4A1 and these miRNAs, we analyzed endogenous expression levels in several pediatric cancer cell lines. NR4A1 was upregulated in RD, Rh41, and Rh30 rhabdomyosarcoma cells and D341 and Daoy medulloblastoma cells as well as NB3 neuroblastoma cells. All three miRNAs were downregulated in Daoy cells. Considering that miR-124 is highly expressed in the brain and is a tumor suppressor, we decided to investigate the functional significance between NR4A1 and miR-124 in Daoy cells. We found that miR-124 could decrease NR4A1 mRNA and protein levels as well as the expression of several NR4A1 target genes. Overexpression of NR4A1 led to enhanced cell viability and proliferation while knockdown resulted in the opposite phenotype. Furthermore, stable expression of miR-124 in Daoy cells resulted in decreased proliferation and smaller spheroid formation. Lastly, we examined expression levels in granule neuron precursors (GNPs), which are the most common cell type in the cerebellum where medulloblastoma arises. Interestingly, there was an inverse expression pattern in which miR-124 was increased while Nr4a1 was decreased in the differentiated GNPs, suggesting a potential role for NR4A1 in neuronal development.

In addition to cancer cell proliferation, the role of NR4A1 in skeletal muscle differentiation was also explored. We found that NR4A1 increased during the differentiation of human LHCN myoblasts, and that knockdown of NR4A1 impairs differentiation and reduces expression of myogenic markers in LHCN as well as SkMC, and HSMM primary human skeletal muscle cells. This data agrees with previous studies performed in mouse models and mouse $\mathrm{C} 2 \mathrm{C} 12$ cells showing increased $\mathrm{Nr} 4 \mathrm{a} 1$ expression during differentiation as well as the ability of NR4A1 to enhance muscle mass and myofiber size.

Together, these two studies highlight two different and opposing functional roles of NR4A1 in medulloblastoma and skeletal muscle. The first study identified three miRNAs capable of directly targeting and suppressing NR4A1 and also provides a rationale for the use of miRNA mimics as a potential therapeutic in cancers with high NR4A1 expression. In the second study, we provided evidence that further confirms the pro-myogenic function of NR4A1 during skeletal muscle differentiation. It is important to understand this basic biology as it can help further understand and treat diseases related to muscle such as rhabdomyosarcoma and muscular dystrophy. 


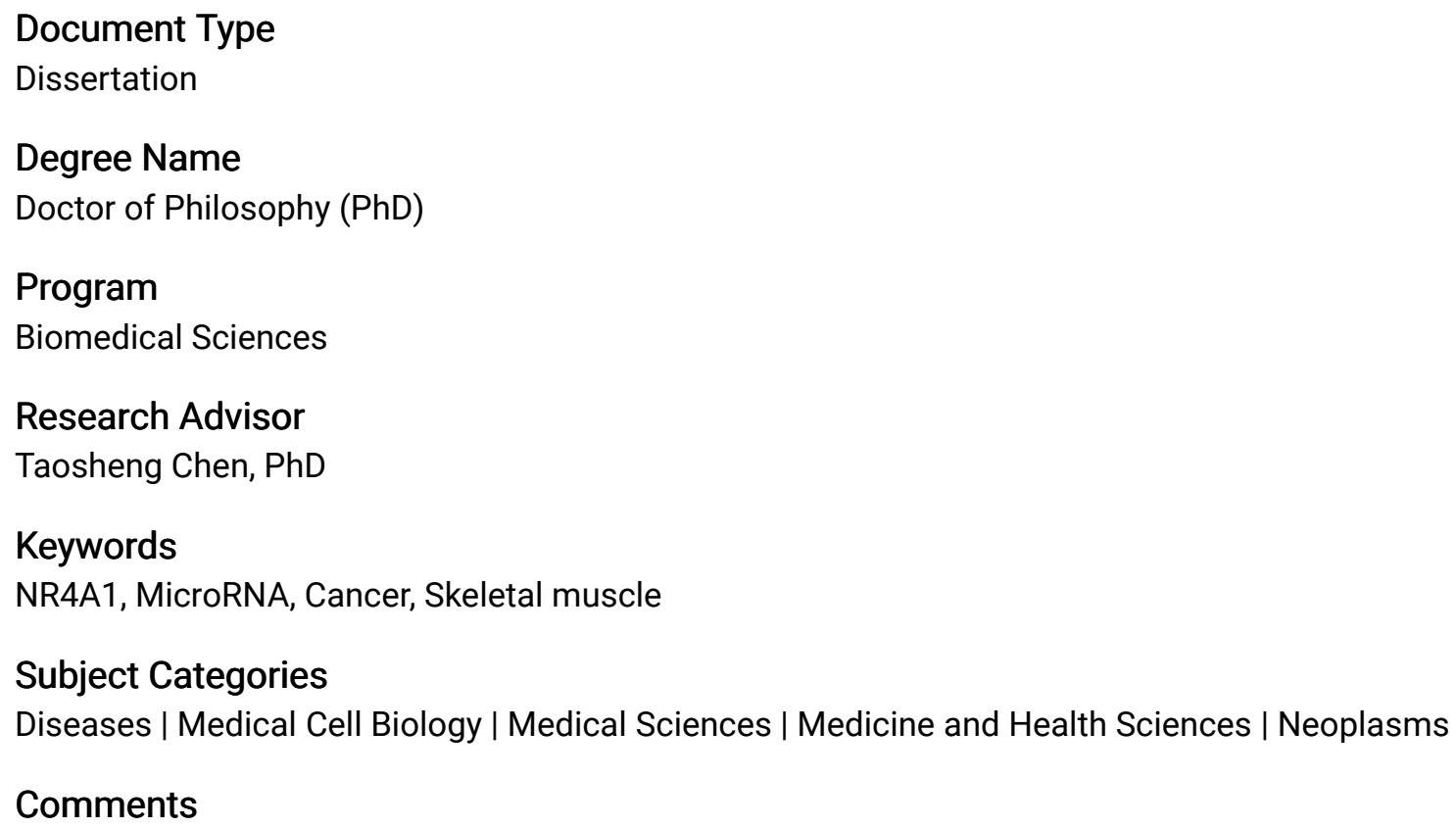


The Roles of Nuclear Receptor NR4A1 in Cancer Cell Proliferation and Skeletal Muscle Differentiation

\author{
A Dissertation \\ Presented for \\ The Graduate Studies Council \\ The University of Tennessee \\ Health Science Center
}

\begin{abstract}
In Partial Fulfillment
Of the Requirements for the Degree

Doctor of Philosophy

From The University of Tennessee
\end{abstract}

By

Alexa Farmer

August 2016 
Portions of Chapters 3 and 5 (c) 2016 by PLOS ONE. All other material (C) 2016 by Alexa Farmer. All rights reserved. 


\section{DEDICATION}

This dissertation is dedicated to my husband and our families for their love and support during my time in graduate school. 


\section{ACKNOWLEDGEMENTS}

Firstly, I would like to thank my mentor, Dr. Taosheng Chen. I am grateful to him for allowing me to rotate and join such a wonderful lab. His encouragement and guidance helped me become an independent, motivated, and critically-thinking scientist. I would like to thank my committee members, Drs. Meiyun Fan, Nick Laribee, Mark Hatley, and Erin Schuetz for their feedback and support of my project. Finally, I would like to thank my lab mates as well as the department of Chemical Biology and Therapeutics at St. Jude Children's Research Hospital for being so friendly and welcoming since my first day. I would especially like to recognize Drs. Jordan Beard, Apana Takwi, Milu Cherian, Jesse Bakke, Su Sien Ong, Yue-Ming Wang, Ayesha Elias, and Jing Wu, as well as Mrs. Jessica Hoyer for all of their help and support over the last 4 years. They were able to make graduate school a much more enjoyable experience. 


\begin{abstract}
Nuclear receptors (NRs) constitute a major class of drug targets in the treatment of various cancer types. NRs respond to cellular signals and become activated upon ligand binding to transcriptionally modulate expression of target genes. NR4A1 (Nur77) is a member of the NR4A family of nuclear receptors and displays an oncogenic profile in many cancer models. It is often upregulated in adult solid malignancies and is known to promote cell proliferation and survival. Knockdown studies of NR4A1 in cancer cell lines result in decreased cell growth and angiogenesis and increased apoptosis, suggesting NR4A1 is an oncogenic protein. Due to the elevated levels of NR4A1 in cancer, it is important to determine the regulatory mechanisms behind this expression pattern.

One such mechanism is through microRNAs (miRNAs), which regulate gene expression by binding to the 3'UTR of target mRNA and effectively inhibit translation into protein. Prior to this study, no miRNAs had been identified to directly target NR4A1. By using luciferase reporter assays, we identified miR-124, miR-15a, and miR-224 as potential NR4A1 regulators. The direct binding of these miRNAs to their potential seed regions within the 3'UTR of $N R 4 A 1$ was confirmed by mutagenesis of their respective seed sequences. This abrogated the binding and thereby confirmed the direct targeting of these miRNAs to these particular sequences. To further study the relationship between NR4A1 and these miRNAs, we analyzed endogenous expression levels in several pediatric cancer cell lines. NR4A1 was upregulated in RD, Rh41, and Rh30 rhabdomyosarcoma cells and D341 and Daoy medulloblastoma cells as well as NB3 neuroblastoma cells. All three miRNAs were downregulated in Daoy cells. Considering that miR-124 is highly expressed in the brain and is a tumor suppressor, we decided to investigate the functional significance between NR4A1 and miR-124 in Daoy cells. We found that miR-124 could decrease NR4A1 mRNA and protein levels as well as the expression of several NR4A1 target genes. Overexpression of NR4A1 led to enhanced cell viability and proliferation while knockdown resulted in the opposite phenotype. Furthermore, stable expression of miR-124 in Daoy cells resulted in decreased proliferation and smaller spheroid formation. Lastly, we examined expression levels in granule neuron precursors (GNPs), which are the most common cell type in the cerebellum where medulloblastoma arises. Interestingly, there was an inverse expression pattern in which miR-124 was increased while $\mathrm{Nr} 4 \mathrm{al}$ was decreased in the differentiated GNPs, suggesting a potential role for NR4A1 in neuronal development.

In addition to cancer cell proliferation, the role of NR4A1 in skeletal muscle differentiation was also explored. We found that NR4A1 increased during the differentiation of human LHCN myoblasts, and that knockdown of NR4A1 impairs differentiation and reduces expression of myogenic markers in LHCN as well as SkMC, and HSMM primary human skeletal muscle cells. This data agrees with previous studies performed in mouse models and mouse $\mathrm{C} 2 \mathrm{C} 12$ cells showing increased $\mathrm{Nr} 4 \mathrm{al}$ expression during differentiation as well as the ability of NR4A1 to enhance muscle mass and myofiber size.
\end{abstract}


Together, these two studies highlight two different and opposing functional roles of NR4A1 in medulloblastoma and skeletal muscle. The first study identified three miRNAs capable of directly targeting and suppressing NR4AI and also provides a rationale for the use of miRNA mimics as a potential therapeutic in cancers with high NR4A1 expression. In the second study, we provided evidence that further confirms the pro-myogenic function of NR4A1 during skeletal muscle differentiation. It is important to understand this basic biology as it can help further understand and treat diseases related to muscle such as rhabdomyosarcoma and muscular dystrophy. 


\section{TABLE OF CONTENTS}

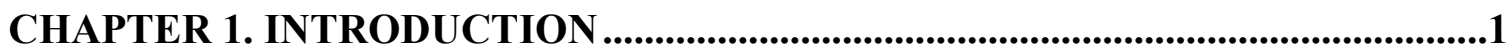

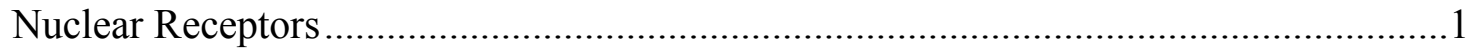

Discovery and classification ..........................................................................

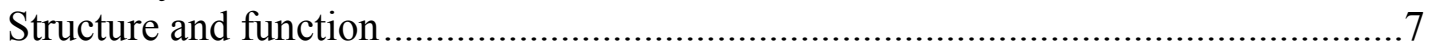

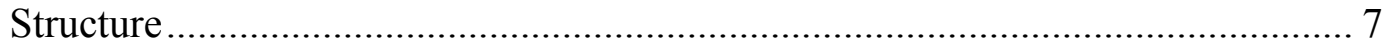

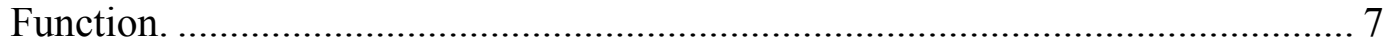

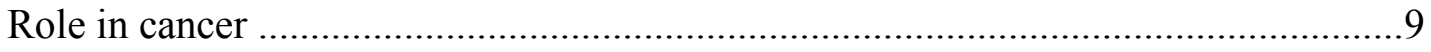

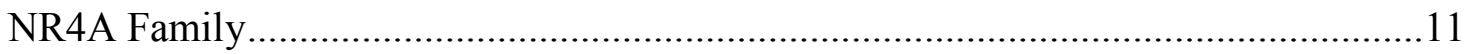

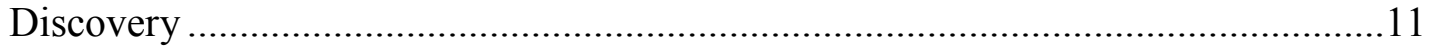

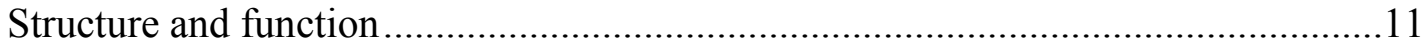

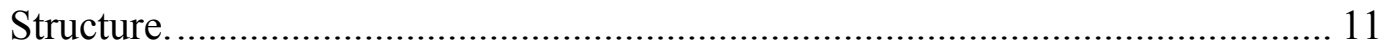

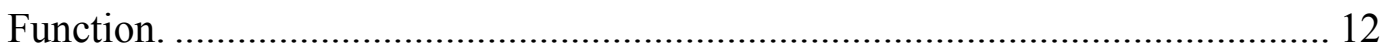

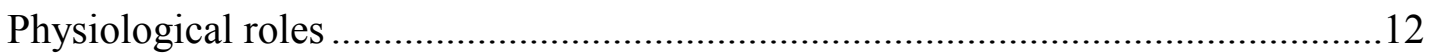

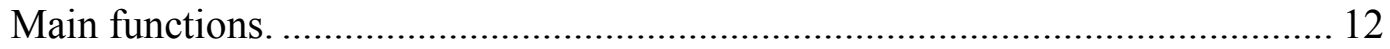

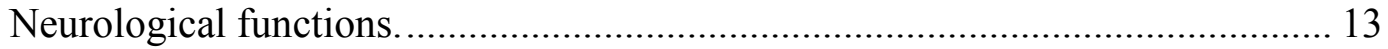

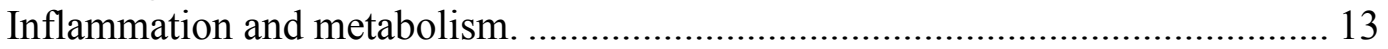

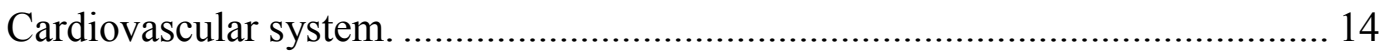

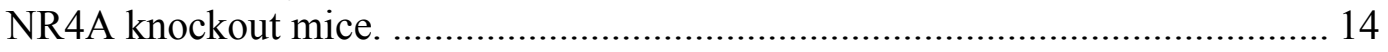

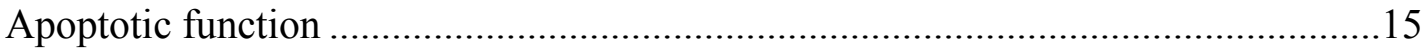

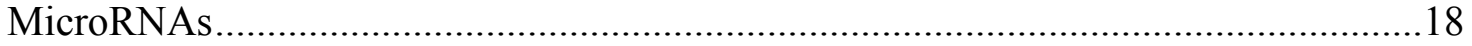

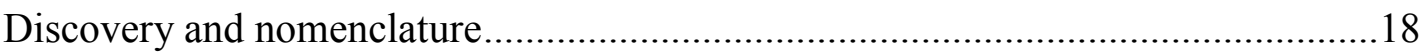

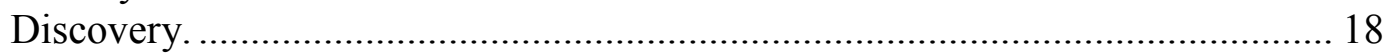

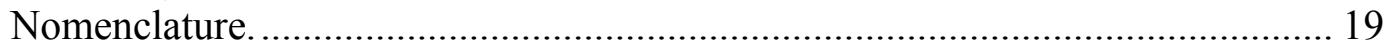

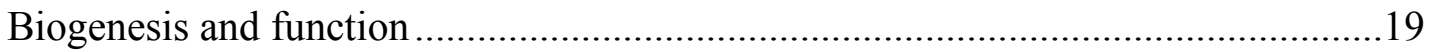

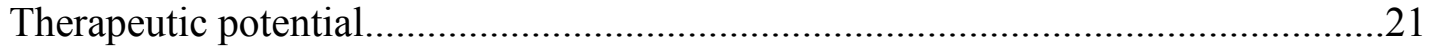

Hypothesis and Specific Aims ................................................................................21

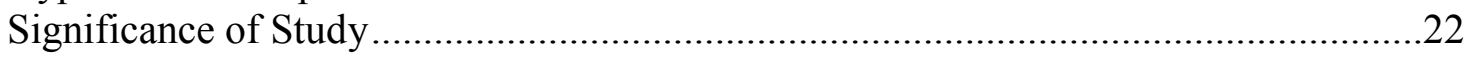

\section{CHAPTER 2. THE REGULATORY EFFECTS OF MICRORNAS ON NR4A1}

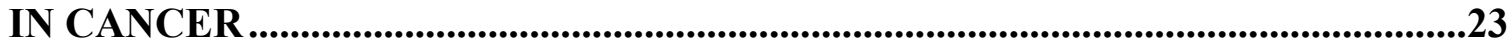

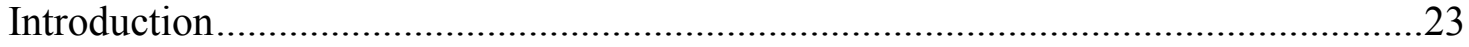

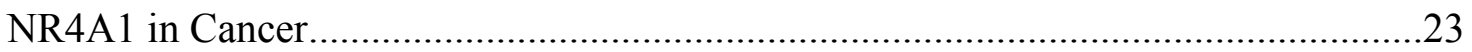

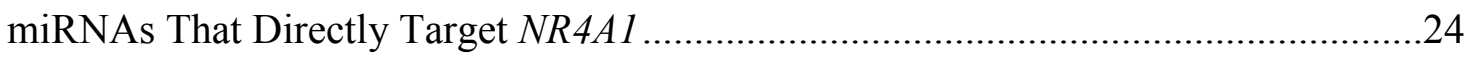

miRNAs That Indirectly Affect NR4A1 Expression and Function ..............................24

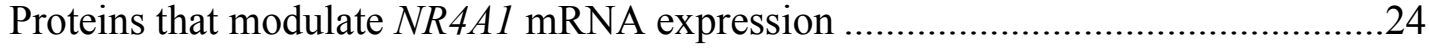

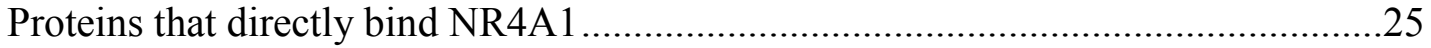

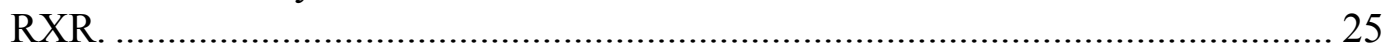

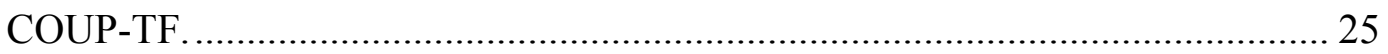

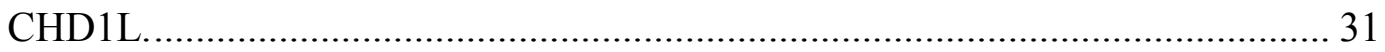

p53

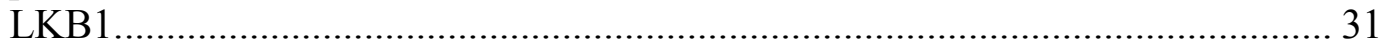

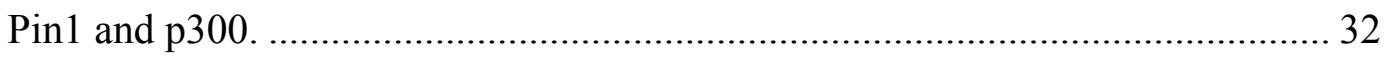

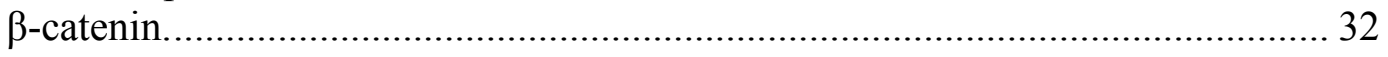




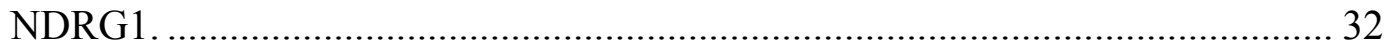

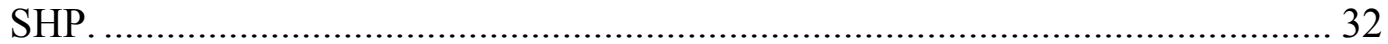

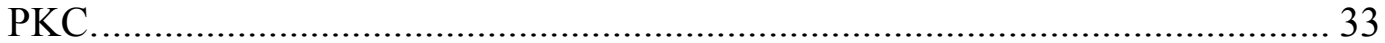

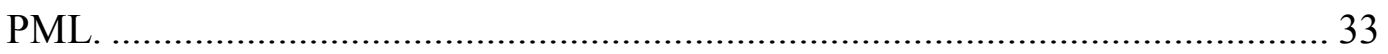

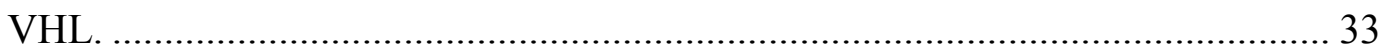

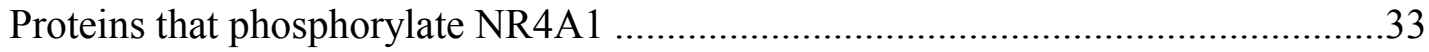

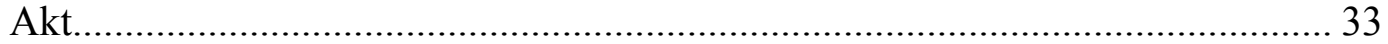

JNK

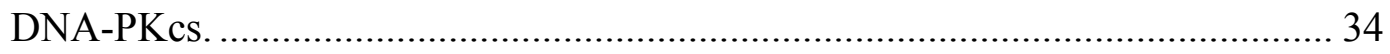

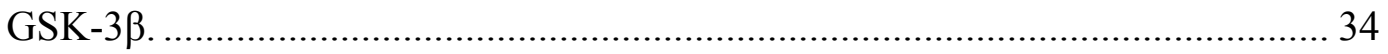

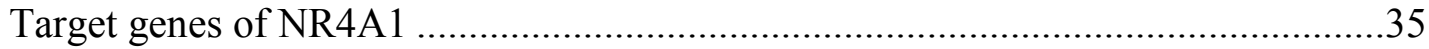

Proliferation and survival genes.................................................................... 35

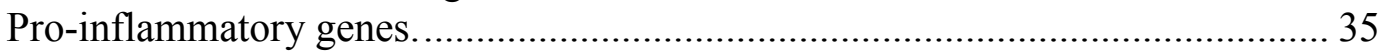

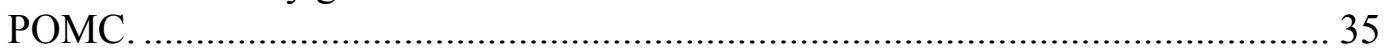

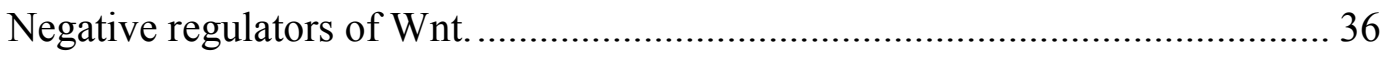

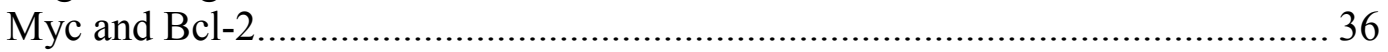

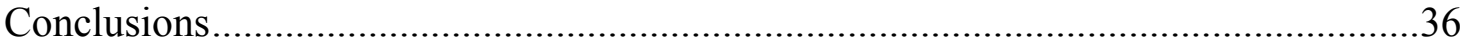

\section{CHAPTER 3. REGULATION OF NUCLEAR RECEPTOR NR4A1 BY}

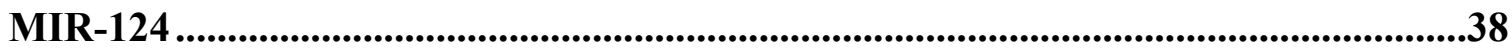

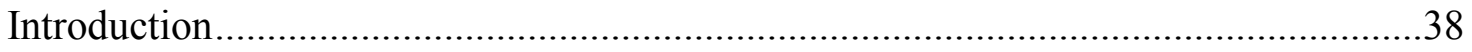

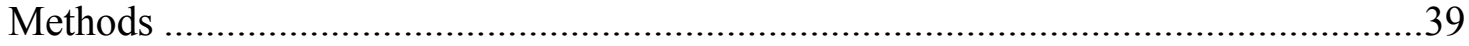

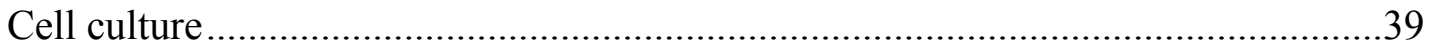

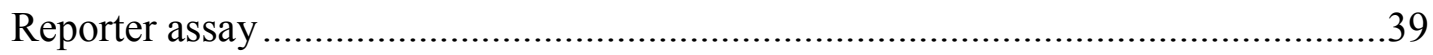

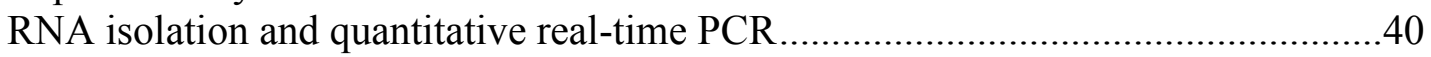

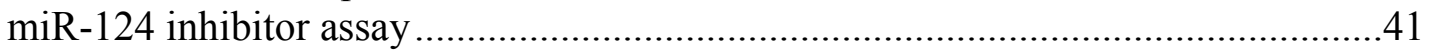

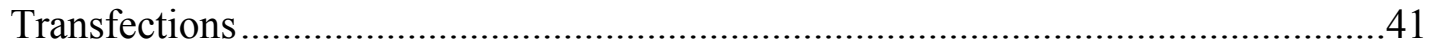

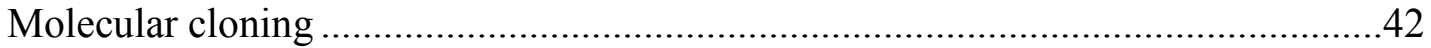

Protein isolation and Western blot analysis ..............................................................42

Lentivirus production and transduction .................................................................43

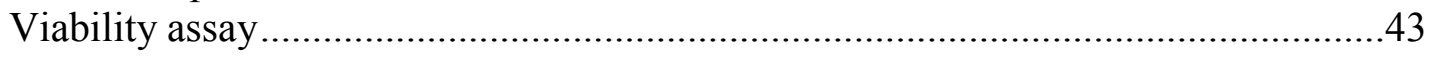

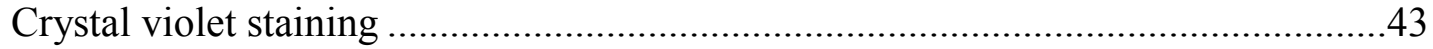

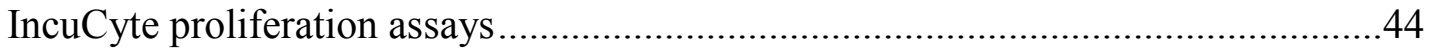

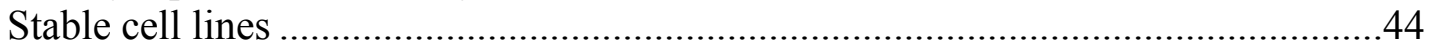

3D-spheroid formation assay …………………………..................................4

Cerebellar granule neuron analysis ...................................................................4

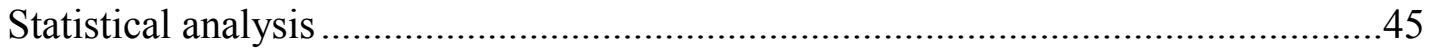

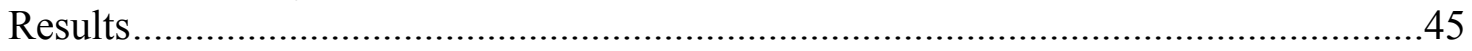

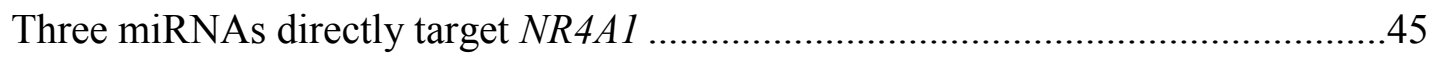

NR4A1 is upregulated in pediatric cancer cell lines..................................................47

miR-124 decreases NR4A1 expression ...............................................................47

NR4A1 promotes cell viability and proliferation ...................................................50

Knockdown of NR4AI decreases cell viability and proliferation ...............................50

miR-124 decreases cell viability and proliferation ...................................................50

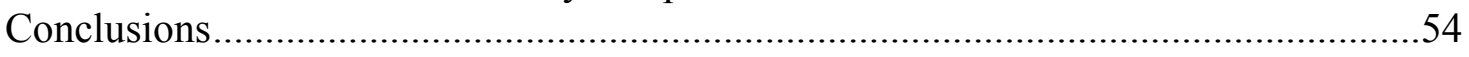




\section{CHAPTER 4. THE ROLE OF NR4A1 IN SKELETAL MUSCLE}

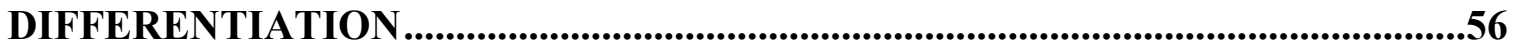

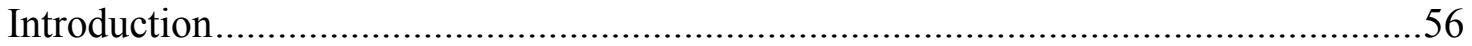

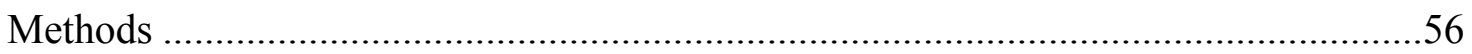

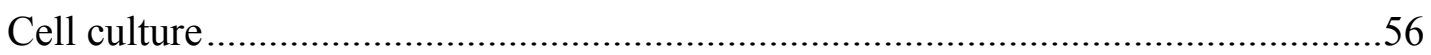

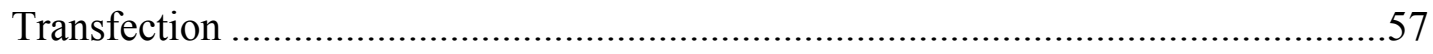

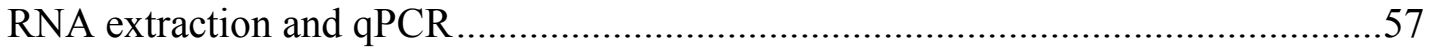

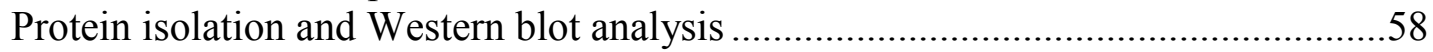

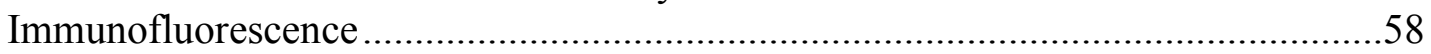

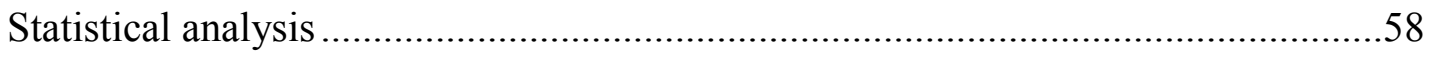

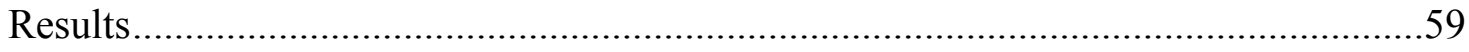

$N R 4 A 1$ expression increases during LHCN myogenesis .....................................59

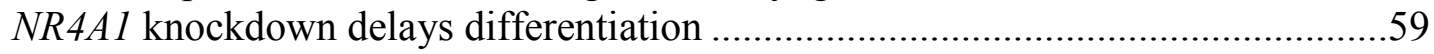

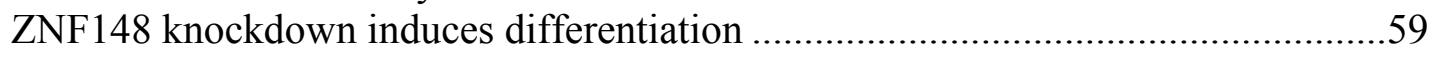

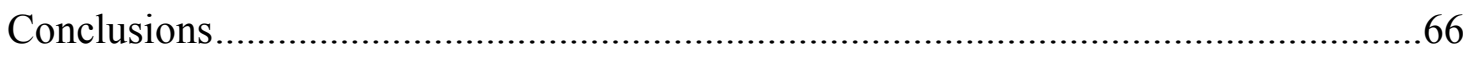

CHAPTER 5. DISCUSSION ............................................................................................69

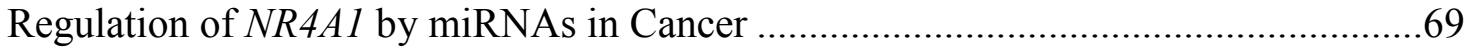

Role of NR4A1 in Skeletal Muscle Differentiation.................................................... 72

LIST OF REFERENCES ......................................................................................77

APPENDIX. SUPPLEMENTAL DATA FOR CHAPTER 3.................................106

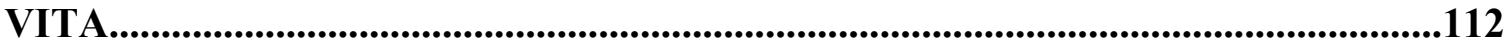




\section{LIST OF TABLES}

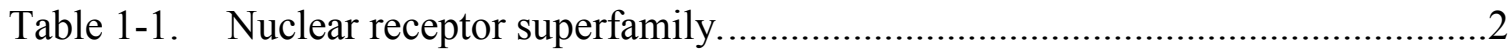

Table 1-2. Compounds and apoptotic stimuli that induce Nur77 to mediate cell death, mainly through mitochondrial localization................................... 17

Table 2-1. Effects of Nur77 regulatory networks on cancer and potential

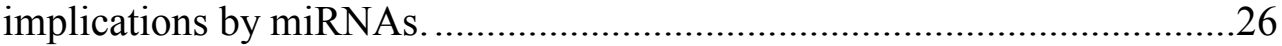




\section{LIST OF FIGURES}

Figure 1-1. The structure and function of liganded and orphan nuclear receptors...........8

Figure 1-2. NR4A1 is induced by cytotoxic agents to mediate cell death. ...................16

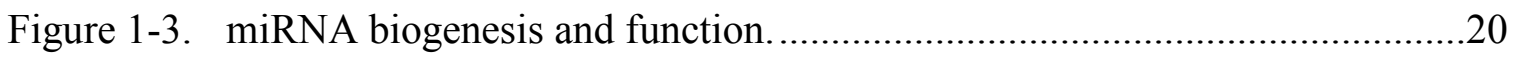

Figure 2-1. NR4A1 mediates cell proliferation and survival in addition to cell death....30

Figure 3-1. miR-124, miR-15a, and miR-224 directly target NR4A1 ........................46

Figure 3-2. NR4A1 is upregulated in pediatric cancer cell lines................................48

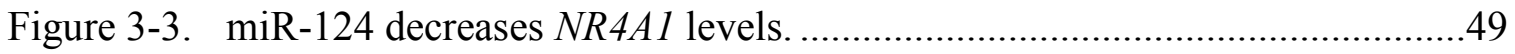

Figure $3-4 . \quad$ NR4A1 promotes cell viability and proliferation. ..................................51

Figure 3-5. NR4A1 knockdown decreases cell viability and proliferation. ....................52

Figure 3-6. miR-124 decreases cell proliferation in 2D and 3D cultures.....................53

Figure 4-1. NR4A1 expression increases during LHCN differentiation. ........................60

Figure 4-2. NR4A1 expression does not increase during SkMC differentiation.............61

Figure 4-3. NR4A1 expression does not increase during HSMM differentiation............62

Figure 4-4. Knockdown of NR4A1 delays differentiation in LHCN...........................63

Figure 4-5. NR4A1 knockdown delays differentiation in SkMC.................................64

Figure 4-6. NR4A1 knockdown delays differentiation in HSMM..............................65

Figure 4-7. Knockdown of ZNF148 rapidly induces differentiation in LHCN.............67

Figure 5-1. Overview of NR4A1 regulation by miR-124 ......................................70

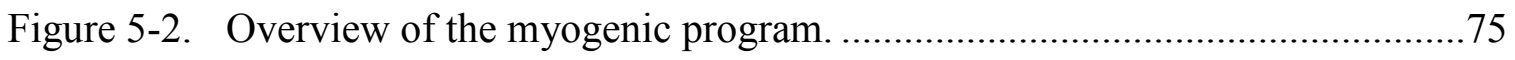

Figure A-1. miR-124 decreases NR4A1 expression in miRNA array. ..........................106

Figure A-2. NR4A1 and miR-124 have inverse expression in granule neurons............108

Figure A-3. An inhibitor of miR-124 increases NR4A1 activity. ............................... 109

Figure A-4. miR-124 decreases levels of NR4A1 target genes in 293T cells...............110

Figure A-5. NR4A1 knockdown decreases cell viability and proliferation. ..................111 


\title{
CHAPTER 1. INTRODUCTION
}

\author{
Nuclear Receptors
}

\section{Discovery and classification}

Before the word 'hormone' was coined in 1905 by Ernest Starling or the word 'receptor' by Paul Erhlich in 1907, not much was known about hormones or nuclear (hormone) receptors [1]. The first NRs cloned were the glucocorticoid and estrogen receptors in 1985 and 1986, respectively [2, 3], although the estrogen receptor was originally isolated in 1958 by Elwood Jensen [4]. Preceding the discovery of these receptors, cortisone, a steroid that binds the glucocorticoid receptor, and thyroxine, an iodoamino acid that binds the thyroid receptor, were the first ligands to be isolated and analyzed for their structures in $1926[5,6]$. Soon after in the 1920s and 1930s, the pancreatic hormone insulin was characterized along with the steroid hormones estrogen, testosterone, and progesterone [1]. It is now known that humans have a total of 48 nuclear receptors termed the nuclear receptor superfamily, as listed in Table 1-1 [7].

Over the years as more and more NRs were discovered, there was much confusion as to the nomenclature of these genes. Hence, in 1999 a group of scientists came together to formally name these receptors based on their phylogeny [8]. These 48 nuclear receptors have since been classified into 6 subfamilies, with the first subfamily, the thyroid hormone receptor-like family, having 8 subgroups. These groupings are based on sequence homology [9-11]. There are also several ways of referring to each receptor. Other than spelling out the full name of the receptor, it can also be identified by its nuclear receptor nomenclature committee (NRNC) symbol, its abbreviation, or its gene name. For example, nerve growth factor-induced clone B, a member of the nerve growth factor IB-like subgroup, is part of the 4th subgroup and is the first member of its family and therefore has the NRNC symbol NR4A1. Its abbreviation is Nur77, although it has many other common names including NGFIB, NR4A1, and TR3, and lastly its gene symbol is NR4A1.

They can also be grouped into liganded NRs, adopted NRs, and orphan NRs. Liganded NRs are those which have a known ligand, such as the estrogen receptor with estrogen acting as its ligand. On the other hand, orphan receptors have no known endogenous ligand, such as COUP-TF. And as the name implies, adopted receptors are those which were once orphans but now have an identified ligand, such as FXR found to be activated by bile acids [12]. The initial discovery of nuclear receptors was based on using ligands to identify the receptor they bind to, but as technology improved it became possible to discover receptors without knowing their ligands, hence the appearance of adopted nuclear receptors. 
Table 1-1. Nuclear receptor superfamily.

\begin{tabular}{|c|c|c|c|c|c|c|}
\hline Subfamily & Group & Name & Abbreviation & Ligand & Disease implicated & Therapeutic drug name \\
\hline \multirow[t]{13}{*}{$\begin{array}{l}\text { Thyroid } \\
\text { hormone } \\
\text { receptor-like }\end{array}$} & $\begin{array}{l}\text { Thyroid hormone } \\
\text { receptor }\end{array}$ & $\begin{array}{l}\text { thyroid hormone } \\
\text { receptor } \alpha\end{array}$ & $\mathrm{TR} \alpha$ & thyroid hormone & $\begin{array}{l}\text { thyroid resistance } \\
\text { syndrome, thyroid } \\
\text { cancer }\end{array}$ & $\begin{array}{l}\text { evothyroxine, } \\
\text { levothyroxine, } \\
\text { liothyronine }\end{array}$ \\
\hline & & $\begin{array}{l}\text { thyroid hormone } \\
\text { receptor } \beta\end{array}$ & $\operatorname{TR} \beta$ & thyroid hormone & hypercholesterolemia & \\
\hline & Retinoic acid receptor & Retinoic acid receptor $\alpha$ & RAR $\alpha$ & retinoic acids & $\begin{array}{l}\text { acute promyelocytic } \\
\text { leukemia, kidney } \\
\text { disease, Alzheimer's } \\
\text { disease, skin diseases, } \\
\text { cancers }\end{array}$ & $\begin{array}{l}\text { all-trans retinoic acid } \\
\text { (ATRA) }\end{array}$ \\
\hline & & Retinoic acid receptor $\beta$ & RAR $\beta$ & retinoic acids & & \\
\hline & & Retinoic acid receptor $\gamma$ & $\operatorname{RAR} \gamma$ & retinoic acids & & \\
\hline & $\begin{array}{l}\text { Peroxisome } \\
\text { proliferator-activated } \\
\text { receptor }\end{array}$ & $\begin{array}{l}\text { Peroxisome } \\
\text { proliferator-activated } \\
\text { receptor } \alpha\end{array}$ & $\operatorname{PPAR} \alpha$ & $\begin{array}{l}\text { Fatty acids and } \\
\text { prostaglandins }\end{array}$ & $\begin{array}{l}\text { Type II diabetes, } \\
\text { atherosclerosis, obesity, } \\
\text { hyperlipidemia }\end{array}$ & $\begin{array}{l}\text { fibrates (clofibrate, } \\
\text { gemfibrozil, fenofibrate) }\end{array}$ \\
\hline & & $\begin{array}{l}\text { Peroxisome } \\
\text { proliferator-activated } \\
\text { receptor } \beta / \delta\end{array}$ & $\mathrm{PPAR} \beta / \delta$ & $\begin{array}{l}\text { Fatty acids and } \\
\text { prostaglandins }\end{array}$ & $\begin{array}{l}\text { Type II diabetes, } \\
\text { atherosclerosis, obesity, } \\
\text { hyperlipidemia }\end{array}$ & \\
\hline & & $\begin{array}{l}\text { Peroxisome } \\
\text { proliferator-activated } \\
\text { receptor } \gamma\end{array}$ & $\operatorname{PPAR} \gamma$ & $\begin{array}{l}\text { Fatty acids and } \\
\text { prostaglandins }\end{array}$ & $\begin{array}{l}\text { Type II diabetes, } \\
\text { atherosclerosis, } \\
\text { obesity, hyperlipidemia, } \\
\text { anaplastic cancer }\end{array}$ & $\begin{array}{l}\text { thiazolidinediones } \\
\text { (roziglitazone, } \\
\text { pioglitazone, } \\
\text { perflurooctanoic acid), } \\
\text { RS544 }\end{array}$ \\
\hline & Rev-ErbA & Reverse-Erb $\alpha$ & $\mathrm{REV}-\mathrm{ERB} \alpha$ & heme & & \\
\hline & & Reverse-Erb $\beta$ & REV-ERB $\beta$ & heme & & \\
\hline & $\begin{array}{l}\text { RAR-related orphan } \\
\text { receptor }\end{array}$ & $\begin{array}{l}\text { RAR-related orphan } \\
\text { receptor } \alpha\end{array}$ & $\mathrm{ROR} \alpha$ & $\begin{array}{l}\text { cholesterol and } \\
\text { ATRA }\end{array}$ & atherosclerosis & \\
\hline & & $\begin{array}{l}\text { RAR-related orphan } \\
\text { receptor } \beta\end{array}$ & $\mathrm{ROR} \beta$ & $\begin{array}{l}\text { cholesterol and } \\
\text { ATRA }\end{array}$ & & \\
\hline & & $\begin{array}{l}\text { RAR-related orphan } \\
\text { receptor } \gamma\end{array}$ & $\mathrm{ROR} \gamma$ & $\begin{array}{l}\text { cholesterol and } \\
\text { ATRA }\end{array}$ & & \\
\hline
\end{tabular}


Table 1-1. Continued.

\begin{tabular}{|c|c|c|c|c|c|c|}
\hline Subfamily & Group & Name & Abbreviation & Ligand & Disease implicated & Therapeutic drug name \\
\hline & Liver X receptor-like & Liver X receptor $\alpha$ & $\mathrm{LXR} \alpha$ & oxysterols & $\begin{array}{l}\text { Alzheimer's disease, } \\
\text { atherosclerosis, } \\
\text { dyslipidemia, non- } \\
\text { alcoholic fatty liver } \\
\text { disease, breast cancer }\end{array}$ & $\begin{array}{l}\text { TO901317, GW3965, N- } \\
\text { Acylthiadiazolines }\end{array}$ \\
\hline & & Liver $\mathrm{X}$ receptor $\beta$ & LXR $\beta$ & oxysterols & $\begin{array}{l}\text { atherosclerosis, } \\
\text { dyslipidemia }\end{array}$ & \\
\hline & & Farnesoid X receptor $\alpha$ & $\mathrm{FXR} \alpha$ & oxysterols, bile acids & $\begin{array}{l}\text { cholestasis, } \\
\text { hypercholesterolemia, } \\
\text { biliary cirrhosis, non- } \\
\text { alcoholic fatty liver } \\
\text { disease }\end{array}$ & $\begin{array}{l}\text { guggulsterone, } \\
\text { chenodeoxycholic acid, } \\
\text { fexaramine }\end{array}$ \\
\hline & & Farnesoid X receptor $\beta$ & FXR $\beta$ & oxysterols & & \\
\hline & $\begin{array}{l}\text { Vitamin D receptor- } \\
\text { like }\end{array}$ & Vitamin D receptor & VDR & $\begin{array}{l}\text { vitamin D and } \\
\text { lithocholic acid }\end{array}$ & $\begin{array}{l}\text { hypocalcemia, } \\
\text { osteoporosis, renal } \\
\text { failure, colon cancer, } \\
\text { diabetic nephropathy, } \\
\text { hypertension, } \\
\text { atherosclerosis }\end{array}$ & calcitriol, doxercalciferol \\
\hline & & Pregnane $\mathrm{X}$ receptor & PXR & $\begin{array}{l}\text { xenobiotics and } \\
\text { endobiotics }\end{array}$ & $\begin{array}{l}\text { cholestatic liver disease, } \\
\text { hyperbilirubinemia, liver } \\
\text { injury, cancer }\end{array}$ & rifampicin \\
\hline & & $\begin{array}{l}\text { Constitutive androstane } \\
\text { receptor }\end{array}$ & CAR & $\begin{array}{l}\text { xenobiotics, } \\
\text { androstane }\end{array}$ & $\begin{array}{l}\text { cholestatic liver disease, } \\
\text { type II diabetes }\end{array}$ & phenobarbitol \\
\hline \multirow[t]{3}{*}{$\begin{array}{l}\text { Retinoid X } \\
\text { Receptor-like }\end{array}$} & $\begin{array}{l}\text { Hepatocyte nuclear } \\
\text { factor- } 4\end{array}$ & $\begin{array}{l}\text { Hepatocyte nuclear } \\
\text { factor- } 4 \alpha\end{array}$ & $\mathrm{HNF} 4 \alpha$ & $\begin{array}{l}\text { fatty acids, palmitic } \\
\text { acid }\end{array}$ & diabetes & \\
\hline & & $\begin{array}{l}\text { Hepatocyte nuclear } \\
\text { factor- } 4 \gamma\end{array}$ & $\mathrm{HNF} 4 \gamma$ & fatty acids & & \\
\hline & Retinoid X receptor & Retinoid X receptor $\alpha$ & $\mathrm{RXR} \alpha$ & $\begin{array}{l}\text { 9-cis retinoic acid } \\
\text { and docosahexanoic } \\
\text { acid }\end{array}$ & $\begin{array}{l}\text { metabolic diseases, } \\
\text { cancers (skin cancer, } \\
\text { cutaneous T cell } \\
\text { lymphoma) }\end{array}$ & bexarotene \\
\hline
\end{tabular}


Table 1-1. Continued.

\begin{tabular}{|c|c|c|c|c|c|c|}
\hline Subfamily & Group & Name & Abbreviation & Ligand & Disease implicated & $\begin{array}{l}\text { Therapeutic drug } \\
\text { name }\end{array}$ \\
\hline & & Retinoid X receptor $\beta$ & $\mathrm{RXR} \beta$ & $\begin{array}{l}\text { 9-cis retinoic acid and } \\
\text { docosahexanoic acid }\end{array}$ & & \\
\hline & & Retinoid X receptor $\gamma$ & $\mathrm{RXR} \gamma$ & $\begin{array}{l}\text { 9-cis retinoic acid and } \\
\text { docosahexanoic acid }\end{array}$ & & \\
\hline & Testicular receptor & Testicular receptor 2 & TR2 & & & \\
\hline & & Testicular receptor 4 & TR4 & & & \\
\hline & TLX/PNR & $\begin{array}{l}\text { Tailless homolog } \\
\text { orphan receptor }\end{array}$ & TLX & & & \\
\hline & & $\begin{array}{l}\text { Photoreceptor cell- } \\
\text { specific nuclear } \\
\text { receptor }\end{array}$ & PNR & & & \\
\hline & COUP/EAR & $\begin{array}{l}\text { Chicken ovalbumin } \\
\text { upstream promoter- } \\
\text { transcription factor I }\end{array}$ & COUP-TFI & & & \\
\hline & & $\begin{array}{l}\text { Chicken ovalbumin } \\
\text { upstream promoter- } \\
\text { transcription factor II }\end{array}$ & COUP-TFII & & & \\
\hline & & V-erbA-related & EAR-2 & & & \\
\hline \multirow[t]{4}{*}{$\begin{array}{l}\text { Estrogen } \\
\text { Receptor-like }\end{array}$} & Estrogen receptor & Estrogen receptor- $\alpha$ & $\mathrm{ER} \alpha$ & estrogens & $\begin{array}{l}\text { breast cancer, ovarian } \\
\text { cancer, prostate cancer, } \\
\text { colon cancer, } \\
\text { osteoporosis }\end{array}$ & $\begin{array}{l}\text { tamoxifen, raloxifene, } \\
\text { gen-estein, } \\
\text { diethylstilbestrol, } \\
\text { equineestrogens }\end{array}$ \\
\hline & & Estrogen receptor- $\beta$ & $\mathrm{ER} \beta$ & estrogens & $\begin{array}{l}\text { breast cancer, ovarian } \\
\text { cancer, prostate cancer, } \\
\text { colon cancer, } \\
\text { osteoporosis }\end{array}$ & \\
\hline & $\begin{array}{l}\text { Estrogen related } \\
\text { receptor }\end{array}$ & $\begin{array}{l}\text { Estrogen-related } \\
\text { receptor- } \alpha\end{array}$ & $\mathrm{ERR} \alpha$ & & & \\
\hline & & $\begin{array}{l}\text { Estrogen-related } \\
\text { receptor- } \beta\end{array}$ & $\mathrm{ERR} \beta$ & & & \\
\hline
\end{tabular}


Table 1-1. Continued.

\begin{tabular}{|c|c|c|c|c|c|c|}
\hline Subfamily & Group & Name & Abbreviation & Ligand & Disease implicated & $\begin{array}{l}\text { Therapeutic drug } \\
\text { name }\end{array}$ \\
\hline & & $\begin{array}{l}\text { Estrogen-related } \\
\text { receptor- } \gamma\end{array}$ & ERR $\gamma$ & & & \\
\hline & 3-ketosteroid receptors & Glucocorticoid receptor & GR & $\begin{array}{l}\text { glucocorticoids, } \\
\text { cortisol }\end{array}$ & $\begin{array}{l}\text { inflammatory diseases } \\
\text { (Inflammatory bowel } \\
\text { syndrome, auto immune } \\
\text { disorder, rheumatoid } \\
\text { arthritis, gout, asthma, } \\
\text { cancer) }\end{array}$ & $\begin{array}{l}\text { dexamethasone, } \\
\text { cortisol, prednisolone, } \\
\text { RU486 }\end{array}$ \\
\hline & & $\begin{array}{l}\text { Mineralocorticoid } \\
\text { receptor }\end{array}$ & MR & $\begin{array}{l}\text { mineralocorticoids } \\
\text { and glucocorticoids } \\
\text { (aldosterone) }\end{array}$ & $\begin{array}{l}\text { heart failure, } \\
\text { hypertension, } \\
\text { cardiovascular disease, } \\
\text { kidney disease }\end{array}$ & $\begin{array}{l}\text { spironolactone, } \\
\text { eplerenone }\end{array}$ \\
\hline & & Progesterone receptor & PR & progesterone & $\begin{array}{l}\text { breast cancer, } \\
\text { endometriosis }\end{array}$ & RU486 \\
\hline & & Androgen receptor & AR & $\begin{array}{l}\text { androgens, } \\
\text { testosterone }\end{array}$ & $\begin{array}{l}\text { prostate cancer, } \\
\text { osteoporosis }\end{array}$ & flutamide, bicalutamide \\
\hline \multirow{3}{*}{$\begin{array}{l}\text { Nerve } \\
\text { Growth } \\
\text { Factor IB- } \\
\text { like }\end{array}$} & NGFIB/NURR1/NOR1 & $\begin{array}{l}\text { Nerve growth factor } \\
\text { induced gene B }\end{array}$ & NGFIB & $\begin{array}{l}\text { unsaturated fatty } \\
\text { acids }\end{array}$ & & \\
\hline & & $\begin{array}{l}\text { Nuclear receptor } \\
\text { related } 1\end{array}$ & NURR1 & & Parkinson's disease & \\
\hline & & $\begin{array}{l}\text { Neuron-derived orphan } \\
\text { receptor } 1\end{array}$ & NOR1 & & & \\
\hline \multirow{2}{*}{$\begin{array}{l}\text { Steroidogenic } \\
\text { Factor-like }\end{array}$} & SF1/LRH1 & Steroidogenic factor 1 & SF1 & phosphatidylinositols & & \\
\hline & & $\begin{array}{l}\text { Liver receptor } \\
\text { homolog-1 }\end{array}$ & LRH-1 & phosphatidylinositols & & \\
\hline $\begin{array}{l}\text { Germ Cell } \\
\text { Nuclear } \\
\text { Factor-like }\end{array}$ & GCNF & $\begin{array}{l}\text { Germ cell nuclear } \\
\text { factor }\end{array}$ & GCNF & & & \\
\hline
\end{tabular}


Table 1-1. Continued.

\begin{tabular}{|c|c|c|c|c|c|c|}
\hline Subfamily & Group & Name & Abbreviation & Ligand & Disease implicated & $\begin{array}{l}\text { Therapeutic drug } \\
\text { name }\end{array}$ \\
\hline \multirow[t]{2}{*}{ Miscellaneous } & DAX/SHP & $\begin{array}{l}\text { Dosage-sensitive sex } \\
\text { reversal, adrenal } \\
\text { hypoplasia critical } \\
\text { region, on chromosome } \\
\text { X, gene } 1\end{array}$ & DAX1 & & & \\
\hline & & $\begin{array}{l}\text { Small heterodimer } \\
\text { partner }\end{array}$ & SHP & & obesity & \\
\hline
\end{tabular}




\section{Structure and function}

Structure. By definition, nuclear receptors are also classified as transcription factors, meaning they can bind the promoters of their target genes to modulate gene expression. The typical structure of a nuclear receptor consists of a well-conserved DNAbinding domain (DBD), a moderately-conserved C-terminal ligand binding domain (LBD), and a highly variable N-terminal region containing a ligand-independent transactivation domain called Activation Function 1(AF-1) as depicted in Figure 1-1A $[3,10,13]$. The N-terminal region interacts with coregulatory proteins such as coactivators that enhance the transcription of NR target genes [14]. A closer look at the DBD reveals two zinc-finger domains as well as the P-box, which is a motif involved in DNA-binding specificity as well as dimerization with other nuclear receptors as homodimers or heterodimers. In addition to the promoter region, nuclear receptors may also bind enhancer and intronic regions of target genes. Nuclear receptors can bind to these regions, or more specifically their response elements, either as monomers, homodimers, or heterodimers, where heterodimers typically include binding with RXR [14]. Orphan receptors typically bind as monomers such as the NR4A family. However, there are two nuclear receptors, DAX1 and SHP, that surprisingly do not contain a DBD and mainly act as corepressors [15]. There is a flexible hinge region positioned between the DBD and LBD that contains the nuclear localization signal (NLS) responsible for facilitating the import of the receptor inside of the nucleus. The LBD is the largest domain and as its name implies, its function is dependent on the binding of small lipophilic ligands in its hydrophobic ligand binding pocket (LBP) [16]. Although the structure of the LBD is well conserved, the LBP can greatly vary in size. For example, some nuclear receptors such as SF-1 have a large LBP of about $1600 \AA 3$, while some orphan receptors completely lack an LBP $[15,17,18]$. For instance, the orphan receptor NR4A2 contains bulky hydrophobic amino acid side chains in place of its LBP [18]. In addition to the LBP, the LBD also contains a dimerization interface and an additional transactivation domain called Activation Function 2 (AF-2), which interacts with and recruits coregulators and also contains a second NLS [19].

Function. Nuclear receptors function to regulate and facilitate a wide variety of biological processes including metabolism, reproduction, development, aging, and homeostasis [20]. Many of these processes are dependent upon ligands to activate their respective nuclear receptors, which in turn act as intermediates to confer signals to downstream events [19]. Ligands can include a plethora of signaling molecules such as hormones, fatty acids, xenobiotics, cholesterol, vitamin D, steroids, and retinoids [14, 21]. In the case of orphan receptors, which account for about half of all nuclear receptors, these can be induced by many factors such as stress, cytokines, and mitogens [14].

There are three main levels to nuclear receptor function; repression, derepression, and transcriptional activation [19]. In the absence of a ligand, nuclear receptor activity is suppressed by the binding of a corepressor complex, which typically contains histone deacetylase (HDAC) activity. These corepressor complexes commonly contain the subunits SMRT/NCoR2 or NCoR1, which directly bind the receptor [14]. Ligand binding can occur in either the cytoplasm or the nucleus, and receptors bound by ligands in the 
(A)

\section{AF-1 \\ DBD}

(B)

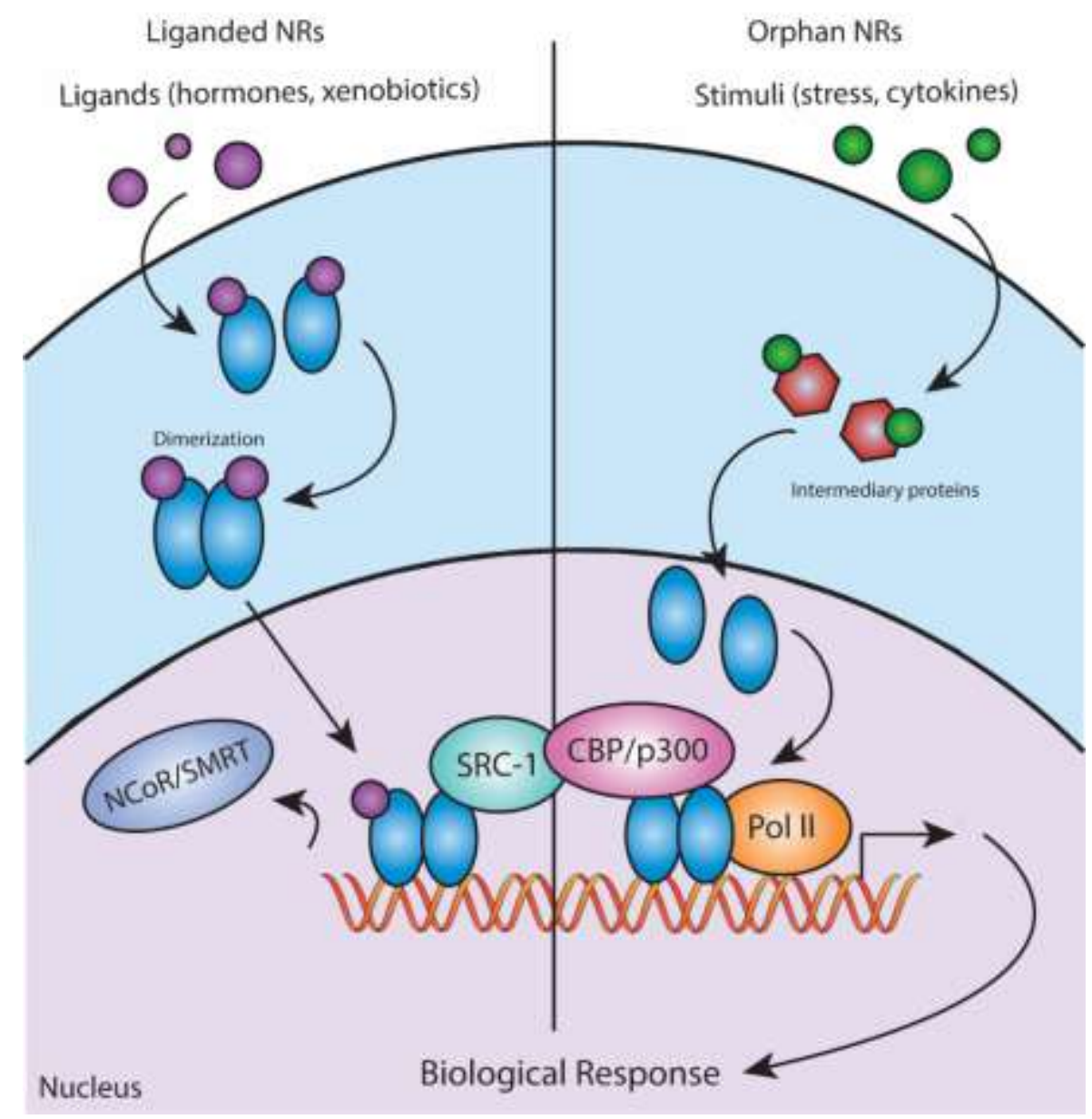

Figure 1-1. The structure and function of liganded and orphan nuclear receptors.

(A) Typical nuclear receptors consist of a DNA-binding (DBD) and ligand-binding domain (LBD) and two transactivation function domains (AF-1 and AF-2) that recruit coregulators. (B) Upon activation by various stimuli, nuclear receptors will heterodimerize and bind to their target genes in conjunction with recruitment of coactivators (SRC-1 and CBP/p300) and release of corepressor (NCoR/SMRT). 
cytoplasm will translocate to the nucleus [22]. Upon ligand binding, the nuclear receptor undergoes a conformational change and derepression occurs in which the corepressor complex is replaced with a coactivator complex containing histone acetyltransferase (HAT) activity. This HAT activity allows for increased transcriptional activity via chromatin decondensation. In the last stage of nuclear receptor function, the coactivator complex is replaced by another coactivator complex and the nuclear receptor is now able to bind with the promoter of its target gene to induce transcriptional activation of that gene via recruitment of transcriptional machinery such as RNA polymerase II as shown in Figure 1-1B [14, 15, 23]. More than 350 coactivators have been identified, including the more common ones like steroid receptor coactivator 1 (SRC-1), steroid receptor coactivator-2 (SRC-2), p300, and cAMP response element-binding protein (CBP) that are likely among the first to be recruited by nuclear receptors [14, 15, 24-26]. On the other hand, nuclear receptors may mediate repression of target gene expression by recruiting corepressors such as the nuclear receptor corepressor (NCoR), silencing mediator for retinoid and thyroid hormone receptors (SMRT), and histone deacetylase 3 (HDAC3) [27-30]. Nuclear receptors can also mediate gene repression by interacting with other transcription factors including activation protein-1 (AP-1) and nuclear factor kappa B (NF-kB) [31, 32]. Typically, unliganded nuclear receptors repress their target genes, while liganded receptors mediate activation of their target genes. Of course, this is a simplistic description of nuclear receptor function. Some nuclear receptors such as orphan receptors may not follow these steps since they may or may not be activated by a ligand [19]. Instead, they may be constitutively active such as the orphan nuclear receptor NR4A1 [33].

In addition to their transcriptional activity, nuclear receptors can also interact with other signaling pathways to impose negative or positive effects on their downstream signaling. Their activity and function can also be affected by post-translational modifications such as phosphorylation, acetylation, methylation, sumoylation, and ubiquitination [15, 34-37]. These interactions can also be dependent on the cell and tissue type.

\section{Role in cancer}

Nuclear receptors are molecular targets for approximately 13\% of FDA-approved drugs [38], and inhibiting this receptor signaling has proved to be beneficial in treating cancer. Since nuclear receptors play a variety of vital roles, disruption or deregulation of their functions can lead to serious consequences. However, their ability to bind ligands makes them therapeutic targets in certain diseases and cancers. Agonists (molecules that enhance receptor function) and antagonists (molecules that disrupt or inhibit receptor function) can play important roles in drug discovery. Certain compounds with agonist/antagonist properties are classified as selective nuclear receptor modulators (SNuRMs), which act as ligands but differ in their activities from the natural endogenous ligands $[15,20]$. More specifically, there are classes of compounds for certain receptors such as selective estrogen receptor modulators (SERMs) for ER, selective androgen receptor modulators for $\mathrm{AR}$, and selective peroxisome proliferator-activated receptor 
modulators for PPAR [39]. The more common nuclear receptors targeted in cancer therapy are the estrogen receptor (ER) in breast cancer, the androgen receptor (AR) in prostate cancer, the retinoic acid receptor (RAR) in acute promyelocytic leukemia [15], and the vitamin $\mathrm{D}$ receptor in breast and colon cancer.

In ER-positive breast cancer, estrogen signaling has been found to promote tumor growth and cancer progression, and treatment with SERMs is used to block this signaling. These modulators function by affecting the recruitment of certain coactivators and corepressors in a tissue-dependent manner [40-42]. For example, the SERMs tamoxifen and raloxifene increase corepressor recruitment in mammary cells, resulting in inhibition of estrogen signaling and decreased breast cancer growth. On the other hand, tamoxifen acts as an agonist in endometrial cells and mediates coactivator recruitment, leading to a higher risk of endometrial cancer [43]. However, raloxifene does not have this agonistic effect in endometrial cells. This is most likely due to preferential binding of coregulators to the AF-1 domain depending on certain factors such as tissue and cell type [44-48]. SERMs can also act as agonists in other tissues including bone, uterine, and cardiovascular tissue $[49,50]$. This kind of tissue selectivity can be beneficial since estrogen signaling can be inhibited in breast cancer while still allowing for normal function in other tissues. This can also be useful in the case of osteoporosis in which raloxifene exerts protective effects while avoiding the increased risk of endometrial cancer that could result from tamoxifen treatment in menopausal women [20,51].

Another major nuclear receptor being targeted in disease is the glucocorticoid receptor (GR) by dexamethasone and prednisolone in inflammatory diseases such as rheumatoid arthritis, immunological disorders, and cancer [20]. These synthetic ligands function as agonists of GR and enhance its ability to disrupt NF-kB and AP-1 activities, leading to decreased proinflammatory cytokines and tumor necrosis factor- $\alpha$, and ultimately resulting in reduced inflammation [52-54]. However, continued treatment with dexamethasone and prednisolone can lead to serious side-effects including diabetes and osteoporosis [55].

Additional receptor targets include PPAR $\alpha$ by fibrates (clofibrate, gemfibrozil, fenofibrate) in hyperlipidemia, PPAR $\gamma$ by thiazolidinediones (roziglitazone, pioglitazone, perflurooctanoic acid) in type II diabetes, RXR by bexarotene and alitretinoin in cancer, RAR by all-trans retinoic acid (ATRA) in acute promyelocytic leukemia, and AR by flutamide and bicalutamide in prostate cancer [15, 56-60]. The xenobiotic receptors CAR and PXR can also play a role in cancer by mediating the metabolism of cancer drugs and drug resistance. PXR is commonly used in the pharmaceutical industry to identify dangerous drug-drug interactions when screening for new cancer therapies [61]. Furthermore, agonists of PXR have therapeutic potential in treating cholestatic liver disease and preventing hyperbilirubinemia [22,62]. Future drug targets include FXR in nonalcoholic steatohepatitis and cholestatic liver disease and LXR in atherosclerosis and Alzheimer's disease by using agonists of these receptors [30, 61, 63]. Many other nuclear receptors are therapeutic targets in various diseases as listed in Table 1-1 [59, 64, 65]. 


\section{NR4A Family}

\section{Discovery}

The NR4A family consists of NR4A1 (Nur77, NGFI-B, TR3), NR4A2 (Nurr1), and NR4A3 (Nor-1). They were first characterized as immediate-early response genes induced by nerve growth factor in PC12 cells [66, 67]. NR4A1 was first isolated in 1989 by Chang et al. from human prostate in which they found that NR4A1 was a $64 \mathrm{kDA}$ receptor protein that could bind to DNA [68]. This was following the discovery of $\mathrm{Nr} 4 \mathrm{a} 1$ in the mouse since Chang et al. noted that human NR4A1 had 86\% nucleotide and $91 \%$ amino acid sequence homology to mouse Nr4a1 [68]. Shortly thereafter, $\mathrm{Nr} 4 a \mathrm{a}$ was identified and cloned from a mouse brain cDNA library by Law et al. and subsequently from fetal human tissue [69-72]. Nr4a2 was found to be highly expressed in the brain compared to other tissues, suggesting an important role for NR4A2 in the central nervous

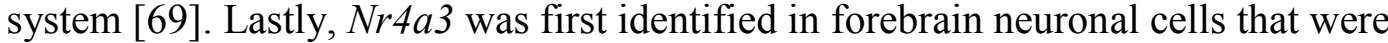
undergoing apoptosis. NR4A3 appropriately stands for neuron derived orphan receptor and was found to encode a $68 \mathrm{kDa}$ protein [73].

\section{Structure and function}

Structure. As mentioned, the NR4A family has been classified as orphan receptors due to the lack of endogenous ligands. However, a recent report claims they are no longer orphans since there are both synthetic and natural compounds that have been shown to bind the LBD of all three NR4As [74]. Additionally, unsaturated fatty acids (UFAs) have been found to act as natural endogenous ligands by binding to the LBD of NR4A1 and NR4A2 [75, 76]. Interestingly, the LBDs of NR4A1 and NR4A3 contain hydrophilic surfaces as opposed to the hydrophobic LBP of liganded nuclear receptors $[77,78]$. In addition, the LBDs of the NR4A family completely lack an LBP and classical coactivator binding site and instead have bulky hydrophobic side chains in place of an LBP where agonists would normally bind [18, 79]. For this reason, it was believed there could be no ligand for the NR4As, though this theory has been disproven by the discovery of small-molecule NR4A1 agonists [75]. Since NRs commonly undergo conformational changes upon ligand binding, it is possible that the NR4As reveal a binding pocket in response to ligand interactions. This was proven to be true for NR4A1 in which the UFA arachidonic acid induced a conformational change, allowing it to bind to the LBD of NR4A1 [75]. In addition, arachidonic acid was found to preferentially bind to NR4A1 oligomers as opposed to monomers and appeared to stabilize these oligomers [75].

In terms of sequence homology between the NR4A members, their DBDs and LBDs are well conserved with about $91-95 \%$ homology in the DBD and about $60 \%$ in the LBD, however the N-terminal containing the AF-1 domain is highly divergent [70, 80, 81]. This higher level of variation within the AF-1 domain likely recruits different cofactors for each NR4A in response to stimuli, which may explain the differential 
binding of the NR4As to their target gene promoters, thereby resulting in the unique responses and functions of each NR4A receptor. More specifically, cofactors have been shown to bind NR4A1 protein between helices 11 and 12 in a hydrophobic region [33, $82,83]$. In terms of the NR4A1 gene structure, it is known to have seven exons, with the AF-1 domain in exon 2, the DBD in exons 3 and 4, and the LBD in exons 5 to 7 [70]. The recruitment of coregulators has been shown to involve the AF-1 domain to mediate NR4A-dependent transcription [33, 77, 78, 84, 85].

Function. Like all nuclear receptors, the NR4As function as transcription factors and bind the promoters of their target genes to modulate their expression in response to certain stimuli. Previously characterized as orphans, it was thought that they act independently of ligands to constitutively modulate gene expression [33, 81, 86-88], however this may no longer be the case since ligands such as UFAs have been discovered to bind the LBD of NR4A1 [75]. They can bind as monomers, homodimers, or heterodimers with each other or with RXR [81, 89-92]. The specific sequence they bind as monomers is called the NGFI-B response element (NBRE) and consists of 5 'A/TAAAGGTCA [93-95]. However as homodimers and NR4A heterodimers, they bind the Nur-responsive element (NurRE), which consists of a sequence motif found naturally occurring in the pro-opiomelanocortin (POMC) promoter [90]. Of the NR4A family, only NR4A1 and NR4A2 form heterodimers with RXR, which bind to the retinoic acid response element DR5 to mediate the function of RXR in retinoid signaling [91, 92].

The NR4A family is classified as immediate early response genes and can be induced by a wide variety of physiological signaling molecules to mediate their functions in proliferation, metabolism, inflammation, differentiation, apoptosis, survival, and DNA repair, among other functions $[33,96,97]$. They have an innate ability to respond quickly to these various stimuli, which include growth factors, stress, fatty acids, hormones, cytokines, neurotransmitters, prostaglandins, phorbol esters, calcium, and various apoptotic signals [33, 98-109]. In addition, they can also be induced by membrane depolarization, magnetic fields, and mechanical stress [33, 104, 110-112]. Importantly, the effects of the NR4A family are extremely context dependent and can vary depending on the specific tissue and cell type as well as experimental conditions. Moreover, their functions are heavily influenced by their expression levels, subcellular localization, posttranslational modifications, interactions with other transcription factors, and crosstalk with many signaling pathways [97].

\section{Physiological roles}

Main functions. The NR4A family is expressed in a variety of tissues, mainly in those that require higher levels of metabolism and energy. These include skeletal muscle, adipose, kidney, liver, T-cells, heart, and the brain [33, 92, 113-116]. The first major function identified for NR4A1 was its requirement for T-cell receptor (TCR)-mediated apoptosis in immature thymocytes and T-cell hybridomas [117, 118]. Both NR4A1 and NR4A3 are highly expressed during TCR-mediated cell death [117, 118], and inhibiting NR4A1 effectively blocks apoptosis in T-cell hybridomas. The lack of NR4A1 in 
transgenic mice can also prevent the process of negative selection $[119,120]$. In contrast, the presence of wild type Nr4a1 or Nr4a3 in mice enable cell death in thymocytes, resulting in reduced numbers of thymocytes and mature T cells [119-121]. A proposed mechanism by which NR4A1 mediates this process is via transcriptional activation of pro-apoptotic genes including Fas-ligand (FasL) [122]. In addition, both NR4A1 and NR4A3 may localize to the mitochondria and interact with Bcl-2 to induce apoptosis during the negative selection of $\mathrm{T}$ cells $[97,123]$. Furthermore, calcium signaling was also found to be required for NR4A1-mediated apoptosis in T cells [124].

Compared to NR4A1, not as much is known about the physiological functions of NR4A2 and NR4A3. However, it is known that NR4A2 plays an important role in the synthesis of dopamine by mediating the transcriptional activation of tyrosine hydroxylase, an enzyme essential for dopamine synthesis [125]. Furthermore, NR4A2 is crucial to the normal development of the midbrain by ensuring the proper synthesis of dopaminergic neurons and facilitating neurotransmitter identity [126]. Interestingly, NR4A2 has been implicated in Parkinson's disease due to genetic mutations [127].

Neurological functions. In addition to the role of NR4A2 in dopamine synthesis, the NR4As appear to have neuroprotective functions as well as roles in learning as they are induced in the hippocampus during fear conditioning in mice [128]. In addition, NR4A1 was found to be required for object location while NR4A2 was important for recognition, long term memory, and object location [129]. NR4A1 has also been implicated in synaptic remodeling as well as a disease resulting from antipsychotic drugs $[74,130,131]$. Furthermore, exogenous NR4A1 expression can mediate the repair of damaged neurons after stroke wherein NR4A1 is normally decreased in neural cells lacking oxygen and glucose [132].

Inflammation and metabolism. The NR4A family has been found to play roles in steroidogenesis by regulating the expression of genes involved in the hypothalamicpituitary-adrenal (HPA) axis, a complex network of glands important for many bodily functions including digestion, mood, and the immune system [133-135]. For example, corticotropin releasing hormone $(\mathrm{CRH})$ and POMC expression are enhanced by NR4A1, resulting in activation of the HPA axis and downstream adrenal glucocorticoid synthesis [135]. The NR4As may also have roles in muscle, adipose, and macrophages as they are induced by various stimuli in these tissues. For example, all three NR4A receptors are rapidly induced by lipopolysaccharide (LPS) and more slowly induced by IFN $\gamma$ in macrophages while only NR4A1 expression is enhanced by oxidized lipids and cytokines in these cells $[136,137]$. Although NR4A1 is induced by inflammatory stimuli, it appears to play a protective role during inflammation as demonstrated by its involvement in $\mathrm{T}$ cell development, Treg cell differentiation, and its anti-inflammatory effects in macrophages [138-140].

The NR4As are also induced in skeletal muscle during recovery from intense exercise [141]. NR4A1 is important for energy expenditure since knockdown of NR4A1 in $\mathrm{C} 2 \mathrm{C} 12$ skeletal muscle cells resulted in decreased expression of genes involved in lipolysis and lipid homeostasis [142], suggesting a role for NR4A1 in treating obesity. 
Similarly, the NR4As are induced during the differentiation of adipocytes, however their expression does not appear to be a requirement for differentiation to occur $[96,143]$. These studies indicate that the NR4A family is important in regulating lipid and glucose metabolism, implicating them in the treatment of metabolism disorders.

Furthermore, the NR4A family was found to be upregulated in the livers of type I and type II diabetic mice [144, 145]. Knockout of NR4A1 in the liver of type II diabetic mice brought the elevated levels of glucose back to almost normal levels and also resulted in decreased expression of gluconeogenic genes [144, 145]. Consistent with this finding, overexpression of NR4A1 induced genes involved in gluconeogenesis and enhanced hepatic glucose production in mice [96]. NR4A1 overexpression can also increase LDL cholesterol and decrease HDL cholesterol while reducing triglyceride levels in the liver [146]. Other studies have shown that NR4A1-deficient mice develop insulin resistance in their skeletal muscle when fed a high-fat diet [147]. These mice also have decreased gene expression related to glucose utilization in skeletal muscle, whereas NR4A1 overexpression in $\mathrm{C} 2 \mathrm{C} 12$ cells results in increased expression of genes involved in glucose and glycogen metabolism $[147,148]$. This indicates a role for the NR4A family in the promotion of type II diabetes.

Cardiovascular system. In addition, the NR4As play roles in energy homeostasis and the vascular system. All three NR4As had increased expression in brown adipose tissue (BAT) during cold exposure, and NR4A1 is induced after $\beta$-adrenergic stimulation in brown adipocytes, although $\mathrm{Nr} 4 \mathrm{a} 1$ deficient mice do not display abnormal nonshivering thermogenesis [149]. This lack of NR4A1 may be compensated by the potential redundancy of NR4A3 since NR4A3 is also induced in brown fat during cold exposure [149]. Interestingly, tissue-specific knockdown of $\mathrm{Nr} 4 a 3$ in the third cerebral ventricle in mice resulted in decreased food consumption and body weight, suggesting a role for the NR4As in energy homeostasis by affecting food intake [96]. In terms of the vascular system, the NR4As are expressed in atherosclerotic lesions and have been shown to regulate genes involved in vascular remodeling processes such as proliferation and inflammation [96]. NR4A1 appears to play a protective role in cardiovascular disease since mice fed a high fat diet experienced decreases in atherosclerotic plaque formation, inflammation, and hepatic lipid deposition in response to NR4A1 overexpression [150152]. In contrast, $\mathrm{Nr} 4 a 3$ knockdown in mice decreases hypercholesterolemia-induced atherosclerosis, suggesting that NR4A3 may promote atherosclerosis [153].

NR4A knockout mice. Surprisingly, NR4A1-null mice do not display abnormal thymic and peripheral T cell death $[133,154]$. Overall, they appear healthy with no noticeable phenotype or developmental problems [154]. The hypothalamic and pituitary systems also appear to function normally, as well as steroidogenesis [33, 133]. One explanation is the possible functional redundancy between the NR4A family wherein the other members may compensate for the lack of NR4A1. For example, it is known that NR4A3 is also capable of pro-apoptotic activity in thymocytes, and NR4A2 and NR4A3 also play roles in the HPA axis that could regulate HPA-related genes in place of NR4A1 [33, 123, 134, 135, 154, 155]. However, mice with both Nr4al and Nr4a3 knocked out quickly develop acute myeloid leukemia (AML) and die within 2 to 4 weeks postnatally 
[156]. As a result of this double knockout, there was increased accumulation of hematopoietic stem cells. Expression of $N R 4 A 1$ and $N R 4 A 3$ was also found to be decreased in patients with AML [156].

Interestingly, mice that lack Nr4a2 develop to full-term but die at birth, seemingly due a defect in respiratory function. However, others report it is due to the lack of dopaminergic neurons in the midbrain with the observation of abnormal movements such as difficulty turning when the mice were placed on their backs as well as the inability to suckle [126, 157, 158]. Finally, Nr4a3 knockout mice exhibit inner ear defects with impaired bi-directional circling behavior, which was associated with decreased endolymphatic fluid space in the ear canals $[159,160]$. In addition, $\mathrm{Nr} 4 a 3$-null mice are more susceptible to limbic seizure activity due to excitotoxic glutamate receptor agonists [160]. In contrast, another study found that mice lacking Nr4a3 die around embryonic day 8.5 (E8.5) due to incomplete gastrulation [161].

\section{Apoptotic function}

Of interest is the translocation function of NR4A1 from the nucleus to the mitochondria to induce apoptosis. Although it appears the main function of NR4A1 is to exert pro-oncogenic and anti-apoptotic effects in cancer cell lines, there are several instances in which this is not the case. For example in lung and other cancer cell lines, addition of the retinoid CD437 (AHPN) induces apoptosis in a NR4A1-dependent manner (Figure 1-2) [162-170]. Many other stimuli have also been shown to induce nuclear export of NR4A1 to mediate apoptosis, including 5-fluorouracil, viruses, phorbol ester (TPA), butyrate, cadmium, and cytosporone B (Csn-B), among many others listed in Table 1-2 [67]. These pro-apoptotic compounds may act by either inducing NR4A1 expression or by directly binding to NR4A1. For example, one study shows that the natural product Csn-B (derived from endophytic fungi) is able to directly interact with the LBD of NR4A1 to mediate NR4A1-dependent apoptosis through multiple mechanisms, including both transcription-dependent and -independent functions (Figure 1-2) [171, 172]. Csn-B treatment of gastric cancer cells resulted in enhanced NR4A1 expression, which was due in part to autoregulation in which NR4A1 was able to bind its own promoter to induce expression [171]. NR4A1 was also found to transcriptionally decrease the antiapoptotic protein BRE in response to Csn-B [172-174]. On the other hand, Csn-B induced translocation of NR4A1 to the mitochondria prior to activation of the apoptotic cascade [171]. This was the first evidence of a molecule that could act as a ligand to bind and activate NR4A1.

The mechanism by which NR4A1 mediates apoptosis involves directly binding to the anti-apoptotic protein Bcl-2 at the mitochondria and converting it to a pro-apoptotic protein. This conversion involves a conformational change of $\mathrm{Bcl}-2$ that reveals its proapoptotic BH3 (Bcl-2 homology) domain [175]. Subsequently, cytochrome c is released and the intrinsic apoptotic cascade is evoked [168]. In addition to the mitochondria, NR4A1 may also localize to the endoplasmic reticulum (ER) to mediate apoptosis. Treatment of neuroblastoma cells with retinoid-related compound CD437 (AHPN) 


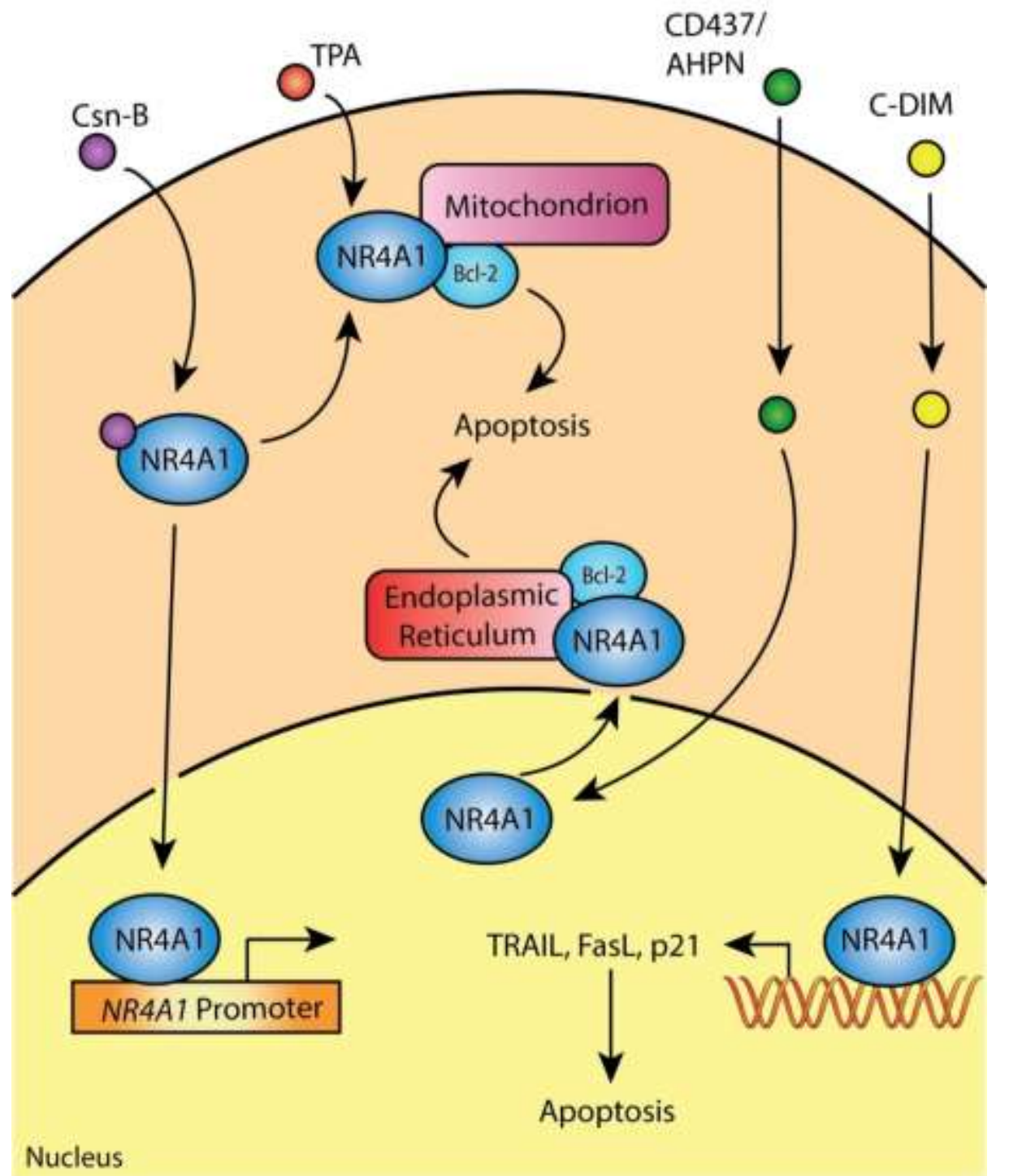

Figure 1-2. NR4A1 is induced by cytotoxic agents to mediate cell death.

NR4A1 can induce apoptosis in response to apoptotic compounds by either translocating to the mitochondria or to the endoplasmic reticulum and converts Bcl-2 to a proapoptotic protein. NR4A1 can also mediate apoptosis by transcriptionally activating genes involved in the cell death program. 
Table 1-2. Compounds and apoptotic stimuli that induce Nur77 to mediate cell death, mainly through mitochondrial localization.

\begin{tabular}{lll}
\hline Inducer & Model system & References \\
\hline $\begin{array}{l}\text { N-butylidenephthalide (BP) } \\
\text { di-n-butyltin dichloride (DBTC) and tri-n-butyltin } \\
\text { chloride (TBTC) }\end{array}$ & HCC & {$[176]$} \\
Synthetic chenodeoxycholic acid derivatives & Gastric cancer & {$[177]$} \\
Cisplatin & Ovarian cancer & {$[178]$} \\
Butyrate & Colon cancer & {$[179]$} \\
Sulindac & Colon cancer & {$[180]$} \\
5-fluorouracil & Colon cancer & {$[180]$} \\
$\begin{array}{l}\text { Phorbol ester 12- O-tetradecanoyl phorbol-13-acetate } \\
\text { (TPA) }\end{array}$ & Prostate and gastric cancer & {$[180]$} \\
Calcium ionophore A23187 & & {$[168,181]$} \\
Etoposide VP-16 & Prostate cancer cells & {$[168]$} \\
AHPN analog 6-[3-(1-adamantyl)-4- hydroxyphenyl]-3- & Prostate and gastric cancer & {$[168,181]$} \\
chloro-2-naphthalenecarboxylic acid (MM11453) & Proster & {$[168]$} \\
Retinoid (Z)-4- [2-bromo-3-(5,6,7,8-tetrahydro- & Prostate cancer & \\
3,5,5,8,8- pentamethyl-2- & & {$[168]$} \\
naphthalenyl)propenoyl]benzoic acid (MM11384) & & \\
Sindbis virus & Primary B cells & {$[182]$} \\
Insulin-like growth factor-binding protein-3 (IGFBP-3) & Prostate cancer & {$[183]$} \\
Shikonin derivatives & Lung and cervical cancer & {$[184]$} \\
Cytosporone B (Csn-B) and related analogues & Gastric cancer & {$[171,173]$} \\
9-cis-retinoic acid & Gastric cancer & {$[170]$} \\
Cadmium & Lung cancer & {$[185]$} \\
CD437 (AHPN) & Neuroblastoma and & {$[186]$} \\
& esophageal squamous & \\
C-DIMs & carcinoma & {$[187,188]$} \\
\hline
\end{tabular}


induced NR4A1 nuclear export and interaction with Bcl-2 at the ER. This interaction led to $\mathrm{Ca}^{2+}$ release from the ER and subsequent ER stress, resulting in apoptosis via ERmediated caspase- 4 activation and ultimately activation of caspase-9 [186].

This apoptotic function of NR4A1 is currently being exploited for its therapeutic potential in cancer. One class of compounds called methylene-substituted diindolylmethanes (C-DIMs) looks especially promising as one group in particular has been studying the utilization of C-DIMs in treating cancer via NR4A1-mediated apoptosis [67]. C-DIMs are small lipophilic molecules synthetically derived from cruciferous vegetables and have been found to inhibit cell and tumor growth in many types of cancers both in vitro and in vivo $[65,170]$. One C-DIM in particular, DIM-C$\mathrm{pPhOCH}_{3}$, induces apoptosis in several cancer types, and knockdown of NR4A1 inhibits this effect in pancreatic, colon, and bladder cancer cells [187, 188]. Several pro-apoptotic genes including TRAIL, FasL, and p21 were also induced, whereas NR4A1 knockdown abrogated this effect [67]. Interestingly, NR4A1 was not exported from the nucleus and did not translocate to the mitochondria during C-DIM-induced apoptosis, suggesting NR4A1 mediates apoptosis via transcriptional activation of these pro-apoptotic genes (Figure 1-2) [67].

On the other hand, the C-DIM analog DIM-C-pPhOH does not activate NR4A1. Instead, it induces apoptosis by preventing NR4A1 from transcriptionally activating antiapoptotic genes such as survivin $[67,187]$. Thus, C-DIMs exhibit anti-tumor properties by acting as both activators and deactivators of NR4A1 and take advantage of the differential activation of target genes dependent on the specific stimuli [67]. It is also possible to synthesize NR4A1 mimics. For example, one group has created a nanopeptide called NuBCP-9 (NR4A1-derived Bcl-2-converting peptide with 9 amino acids), which is capable of inducing apoptosis in cancer models in vitro and in vivo by replicating the action of NR4A1 and converting Bcl-2 to a pro-apoptotic molecule [189]. This NR4A1 mimic would be beneficial in the treatment of breast cancer since this cancer type has higher Bcl-2 expression [172]. Furthermore, the anticancer drug paclitaxel was found to mimic the effects of NR4A1 on Bcl-2 [190]. In all, many anti-tumorigenic compounds can induce NR4A1-mediated apoptosis through various mechanisms including the induction of NR4A1 expression and subsequent mitochondrial or ER targeting (CD437 and TPA), direct binding of compounds (Csn-B) with NR4A1 to activate apoptosis via genomic and non-genomic functions, and either activating or deactivating NR4A1 transcriptional activity (C-DIMs).

\section{MicroRNAs}

\section{Discovery and nomenclature}

Discovery. The first miRNA discovered was lin-4 in Caenorhabditis elegans $(C$. elegans) in 1993 [191]. Seven years passed before the second miRNA (let-7) was identified in 2000, also in C. elegans $[192,193]$. Initially, it was thought that miRNAs 
were specific to $C$. elegans until additional miRNAs were discovered in several other organisms, including humans [194, 195]. To catalog of all the miRNAs identified, a database called miRBase was created, with the most recent update released in 2014 (miRBase 21). This miRNA registry now contains 28,645 hairpin precursor miRNAs, which represents 35,828 mature miRNAs across 223 species (miRBase 21). Of these miRNAs, 1,881 precursors and 2,588 mature miRNAs belong to humans.

Nomenclature. For every miRNA identified, each is assigned a number corresponding to the order in which it was discovered. To specify the organism that the miRNA pertains to, three letters are added before the 'miR', such as hsa-miR-124 for Homo sapiens and mmu-miR-124 for Mus musculus. To denote the mature form of the miRNA, the ' $r$ ' is capitalized (miR-124), whereas a lower case ' $r$ ' (mir-124) indicates both the gene and precursor forms. When mature miRNAs have identical sequences but are processed from different precursor miRNAs, they are differentiated by adding a number to the end of their name, such as miR-124-1 and miR-124-2. Conversely, mature miRNA sequences that differ by one or two nucleotides are denoted by a letter, such as miR-34a and miR-34b. In addition, miRNAs are processed from precursor miRNAs into two complementary mature miRNAs where one is on the 3 ' strand and the other resides on the 5 ' strand. These can be denoted by adding ' $3 p$ ' or ' $5 p$ ', such as miR-124-3p and miR-124-5p. One version may be predominantly expressed over the other, and when the dominant version is known, it keeps the common name (miR-124), whereas the less dominant one is indicated with an asterisk (miR-124*) [196-199].

\section{Biogenesis and function}

miRNAs are non-coding RNAs consisting of 18 to 23 nucleotides that regulate gene expression by binding to the 3' untranslated region (3'UTR) of their target gene and degrading the mRNA, thereby preventing its translation into protein [200, 201]. About half of all miRNAs reside in non-protein coding genes, while the other half are usually within the introns of protein coding transcripts [202]. RNA polymerase II transcribes them into long primary miRNAs (pri-miRNA) that resemble a stem-loop structure, which are then cleaved by the enzyme Drosha before becoming precursor miRNA (pre-miRNA) [203]. These pre-miRNAs are then exported into the cytoplasm via exportin-5 in a RanGTP-dependent fashion where they are further cleaved by Dicer into mature miRNAs, as shown in Figure 1-3 [204-206]. During the final stage of biogenesis, the mature miRNAs consist of two complementary strands that are separated with the more stable passenger strand being degraded and the unstable guide strand being incorporated into the RNAi induced silencing complex (RISC) [195, 207, 208]. This complex is then able to bind the $3^{\prime}$ UTR of its target mRNA in the seed region, which consists of 6 to 8 complementary nucleotides. Although the 3 'UTR is the main target site, miRNAs may also bind the $5^{\prime}$ UTR as well as the coding sequence [209, 210]. 


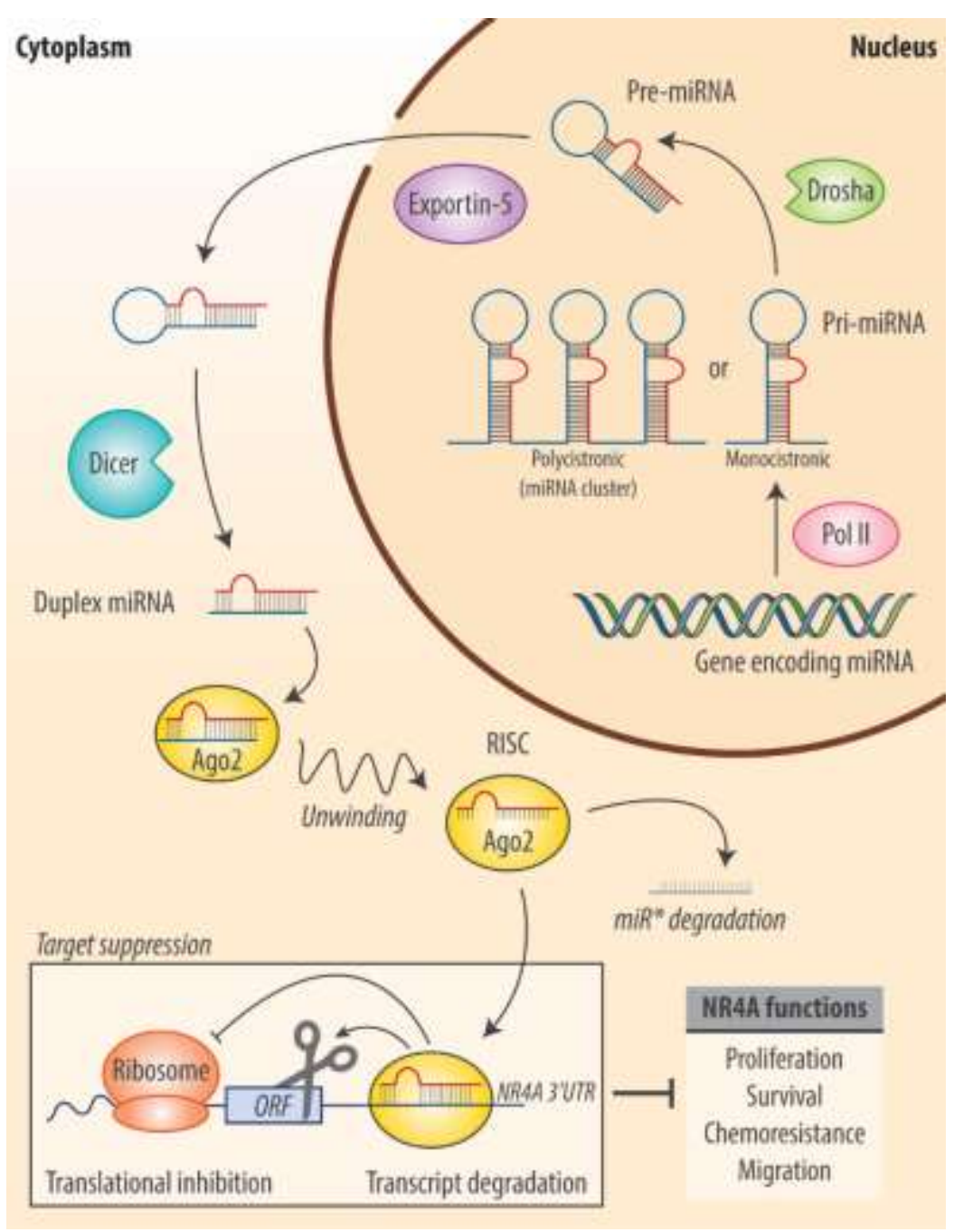

Figure 1-3. miRNA biogenesis and function.

miRNAs are transcribed by RNA polymerase II (Pol II) into either polycistronic or monocistronic pri-miRNA, which are cleaved by Drosha into pre-miRNA. Exportin-5 mediates the translocation of pre-miRNA into the cytoplasm where it is cleaved by Dicer into mature miRNA. A single strand of the mature miRNA is incorporated into the RISC complex, which can then bind to the 3'UTR of target genes to mediate gene suppression. This can lead to inhibition of NR4A oncogenic functions such as proliferation, survival, chemoresistance, and migration. 


\section{Therapeutic potential}

miRNAs are important regulators of almost every cellular process and are estimated to regulate $60 \%$ of the human genome [211]. They are able to form intricate networks wherein a single miRNA can target hundreds of genes, while a single gene may be targeted by numerous miRNAs. This complex regulation has the potential to have profound effects on biological processes. For example, miRNAs have been shown to play important roles in cancer where they can act as tumor suppressors by targeting oncogenes or as oncomiRs by targeting tumor suppressors [212, 213]. Furthermore, many cancers exhibit aberrant miRNA expression where the tumor suppressor-like miRNAs are downregulated, most likely due to hypermethylation of the promoter [214].

In addition to miRNAs acting as biomarkers, they have also seen increased potential for use as therapeutic agents such as miRNA replacement therapy using miRNA mimics [215]. For instance, the miR-34 family harbors strong inhibitory effects on tumor growth in a variety of cancers. It exerts these effects through a number of mechanisms including the direct suppression of mitogenic genes (cyclin E2, E2F3, and CDK4) as well as the anti-apoptotic gene SIRT1, allowing for increased expression of the apoptotic genes p53, p21, and PUMA [216]. In addition, p53 induces miR-34 expression, resulting in a positive feedback loop $[217,218]$. A cancer therapy utilizing the power of miR-34 is currently being tested where a miR-34 mimic is encapsulated in a liposome to allow for more targeted delivery in a range of cancers and is currently being tested in phase I clinical trials. This is the first miRNA to enter clinical trials, and the results thus far appear promising [219]. Many other tumor suppressor-like miRNAs exist with similar effects to miR-34, suggesting the potential for further exploration of miRNA replacement therapy if the miR-34 mimic proves successful.

On the other hand, miRNAs may play an oncogenic role such as the miR-17-92 cluster promoting tumorigenesis in a wide range of cancers [220]. In addition to cancer, miRNAs can have important functions in metabolic disorders, autoimmune diseases, genetic diseases, and infectious diseases [195]. Of importance, the viral replication of hepatitis $\mathrm{C}$ virus (HCV) was found to be dependent on miR-122 [221]. In order to block miR-122 function, a drug was developed (SPC3649) to effectively bind the miRNA in a locked nucleic acid fashion. After successful experimentation in chimpanzees, the drug is currently being tested in phase II clinical trials in humans [222]. If successful, this will be the first miRNA-based therapy for HCV [195].

\section{Hypothesis and Specific Aims}

Studies have shown that NR4A1 is upregulated in many solid tumors, and this overexpression can lead to increased cell proliferation in cancer cell lines, indicating an oncogenic role for this nuclear receptor. Our overall hypothesis is that miRNAs can regulate the expression and downstream function of NR4A1, as depicted in Figure 1-3. We theorize that one of the causes for upregulation of NR4A1 in cancer is due to downregulation of tumor suppressor-like miRNA regulators of NR4A1. 
In addition to this first project, we decided to explore the potential function of NR4A1 during the differentiation of skeletal muscle. Previous studies found that NR4A1 expression increases during skeletal muscle differentiation. Thus, we hypothesize that NR4A1 is playing a pro-myogenic role during this process. To investigate these hypotheses, we proposed the following aims:

1. Elucidate the miRNAs that are directly regulating NR4A1 and their effects on the proliferative function of NR4A1 in pediatric cancer cell lines.

2. Determine the importance of NR4A1 in skeletal muscle differentiation.

\section{Significance of Study}

NR4A1 has been mainly studied in adult cancers, with little research on its role in pediatric cancers. Although we propose to focus on pediatric cancer types, this novel study of the interaction between miRNAs and NR4A1 could have therapeutic potential in both adult and pediatric cancers. NR4A1 is an ideal candidate for a therapeutic target and a potential biomarker in cancer. On the other hand, miRNAs can act as tumor suppressors and are useful as prognostic markers as well as miRNA mimic therapy to treat a variety of cancers.

The discovery of miRNAs that target NR4A1 will add a new aspect to NR4A1 regulation. This study could demonstrate clinical translation through the use of in vivo models in which miRNAs are used as therapy for the pediatric tumors in which NR4A1 is aberrantly amplified. The majority of published research on NR4A1 as a therapeutic target involves utilizing agonists to induce NR4A1-mediated apoptosis, but none have delved into using miRNAs to influence NR4A1 expression and genomic actions.

In addition, our initial studies show that differentiation of skeletal muscle cells is severely delayed upon knockdown of NR4A1. This data supports previous studies showing the importance of NR4A1 in the muscle mass of mice. This information could be useful in terms of cancer therapy since malignancies such as rhabdomyosarcoma (RMS) form as a result of cells failing to differentiate. Furthermore, muscle-related diseases can arise such as muscular dystrophy, a disease that is prevalent in younger populations and is characterized by loss of muscle mass and progressive weakness. Determining the role of NR4A1 during skeletal muscle differentiation may help understand the formation of RMS as well as diseases related to muscle. 


\section{CHAPTER 2. THE REGULATORY EFFECTS OF MICRORNAS ON NR4A1 IN CANCER}

\section{Introduction}

NR4A1, a member of the NR4A family of nuclear receptors, is known for its oncogenic effects in cancer cells. These effects are heavily dependent on its role as a transcription factor to promote expression of genes leading to cell proliferation and survival. The expression and function of NR4A1 are regulated by post-translational modifications and protein-protein interactions, as well as transcriptional regulation by other transcription factors. NR4A1 is aberrantly expressed in many cancers, and one way to therapeutically modulate its expression is through microRNA (miRNA) replacement therapy. This chapter will explore the ways in which miRNAs may be affecting NR4A1 expression and function in the context of cancer. The role of NR4A1 in cancer will first be discussed, followed by the many different ways it may be regulated by miRNAs. Both direct and indirect effects will be detailed since there are not many miRNAs shown to target NR4A1 directly. miRNAs may be targeting NR4A1 indirectly by preventing the expression of genes that would normally interact and regulate NR4A1 via transcriptional regulation, post-translational modifications, and protein-protein interactions. These regulatory networks will be related back to cancer and hypothetical situations will be discussed on how they may affect tumor formation and progression. It is important to understand these networks in order to determine the best possible way for treating cancers in which NR4A1 is aberrantly expressed and plays oncogenic roles.

\section{NR4A1 in Cancer}

In many adult cancers, NR4A1 is overexpressed and appears to play a proliferative role. Bladder [223, 224], breast [187, 225-228], colon [180, 188, 228-231], liver [176, 231, 232], pancreatic [233, 234], prostate [235], ovarian [228, 236], and lung $[167,228,237,238]$ cancer all display increased NR4A1 compared to their normal counterparts, with melanoma [228] expressing the highest levels of NR4A1. The transactivation and DNA-binding domains of NR4A1 appear to be the culprit for its proliferative function in these cancers. Through transcriptional upregulation of genes involved in cell cycle progression and inhibition of apoptosis, NR4A1 can thereby exert its mitogenic effects. For example in melanoma and lung cancer, NR4A1 mediates cell cycle progression and proliferation as well as angiogenesis, which was dependent on its transactivation and DNA binding ability [167, 239]. Furthermore, knockdown studies of NR4A1 in many cancer cell lines resulted in decreased cell growth and angiogenesis and increased apoptosis, providing further evidence for the role of NR4A1 as an oncogenic factor [67]. On the other hand, NR4A1 is downregulated in acute myeloid leukemia (AML), and $\mathrm{Nr} 4 a \mathrm{l} / \mathrm{Nr} 4 \mathrm{a} 3$ knockout mice quickly develop AML, leading to the suspicion that NR4A1 is playing a tumor suppressive role in this blood cancer [156]. This seems to contradict its role in solid tumors, but it is more understandable after realizing that NR4A1 possesses the ability to exert opposing roles in different tissues and cell types. 
NR4A1 can also wield its effects by other means, such as interacting with proteins from a multitude of signaling pathways, as well as phosphorylation by kinases to either promote or inhibit its translocation to the cytoplasm. As discussed below, miRNAs also factor into this regulation of NR4A1 activity by both direct and indirect effects, thereby affecting cancer progression in a myriad of ways.

\section{miRNAs That Directly Target NR4A1}

Currently there is a shortage of miRNAs that have been proven to target NR4A1. According to miRTarBase there are no miRNAs that have been validated to target NR4A1 using strong evidence consisting of reporter assays, western blots, and qPCR. There are a total of 6 miRNAs shown to target $N R 4 A 1$ using less strong evidence such as microarrays and next-generation sequencing (NGS). For example, one study used a microarray and found that HeLa cells transfected with miR-124 had decreased NR4A1 mRNA [240]. Another study also used a microarray and showed that rno-miR-290 targets Nr4al in the rat brain [241]. However, there is one recent study that does validate the targeting of NR4A1 by miR-124, miR-15a, and miR-224 through the use of reporter assays, western blot, and qPCR. This study also found that miR-124 can suppress the transcriptional activity of NR4A1 in Daoy medulloblastoma cells. When miR-124 was exogenously expressed in these cells, proliferation and viability were significantly decreased [242]. Although miR-124 is predicted to target many other genes, it is possible that it is causing these anti-tumor effects through the suppression of NR4A1. miR-124 is an interesting miRNA because it is the most abundant miRNA in the brain with a role in neuronal differentiation. It is a tumor suppressor in many cancer types, several of which express aberrantly low levels of this miRNA. Since NR4A1 is commonly upregulated in cancer, it is easy to see how this aberrant upregulation could be due to the downregulation of miRNAs that target it such as miR-124.

\section{miRNAs That Indirectly Affect NR4A1 Expression and Function}

\section{Proteins that modulate $N R 4 A 1$ mRNA expression}

There are several ways in which miRNAs may indirectly affect NR4A1 expression and function. One way is by targeting proteins that modulate $N R 4 A 1 \mathrm{mRNA}$ expression. For example, the histone acetyltransferase p300 can acetylate $N R 4 A 1$, thereby enhancing its expression in HepG2 and HeLa cells [243]. In addition, the transcription factor AP-1 was shown to bind the promoter of NR4Al and induce its expression in colon cancer cells after exogenous $\beta$-catenin expression [230]. $\beta$-catenin was also found to increase NR4A1 transcription through hypoxia-inducible factor 1-alpha (HIF-1 $\alpha$ ) in colon cancer cells in response to hypoxic conditions [244]. $\beta$-catenin is aberrantly activated in $90 \%$ of colon tumors and acts as an oncogene in this cancer [230], therefore it would appear that $\beta$-catenin is exerting its proliferative effects partly through its induction of $N R 4 A 1$. HIF- $1 \alpha$ was also found to bind to the promoter of $N R 4 A 1$ and promote its 
expression in several cancer cell types including renal cell carcinoma, neuroblastoma [245], hepatocellular carcinoma (HCC), cervical cancer, and breast cancer [246]. EP300 (encodes p300), FOS and $J U N$ (comprises AP-1), CTNNB1 (encodes $\beta$-catenin), and HIF1A are targeted and repressed by many miRNAs as listed in Table 2-1. Upon repression of these genes by miRNAs, NR4Al expression would also theoretically decrease. On the other hand, HDACl is able to repress NR4A1 in HepG2 and HeLa cells [243]. Therefore any miRNAs that target and suppress $H D A C l$ would result in enhanced NR4Al expression.

\section{Proteins that directly bind NR4A1}

RXR. Another way miRNAs may indirectly affect NR4A1 is by targeting binding partners of NR4A1 that in turn affect its localization and function, as depicted in Figure 2-1. One of the most complex interactions may be with the retinoid $\mathrm{X}$ receptor (RXR). Depending on experimental conditions and external stimuli, RXR may heterodimerize with NR4A1 and either bind to DNA to modulate gene expression or localize to the mitochondria to induce apoptosis. When prostate and lung cancer cells were treated with apoptosis-inducing agents 3-Cl-AHPC and TPA (12-O-tetradecanoyl13-phorbol acetate), RXR $\alpha$ formed a heterodimer with NR4A1 and translocated to the mitochondria to induce apoptosis [162]. However, translocation of this heterodimer and subsequent apoptosis were inhibited by the RXR ligand 9-cis-retinoic acid (9-cis-RA) [162]. This RXR ligand along with RXR-selective retinoids, SR11246 and SR11345, strongly promote $\mathrm{RXR} / \mathrm{NR} 4 \mathrm{~A} 1$ heterodimer binding to the retinoic acid response element $\beta R A R E[91,162,247,248]$, although NR4A1 can also increase RA response element transcription independent of retinoic acid [247]. Contrary to this finding, one group found that 9-cis-RA actually enhanced RXR/NR4A1 dimerization and translocation to the mitochondria, along with subsequent apoptosis [249]. This group also found that NR4A1 binding to RXR could suppress p300-mediated RXR acetylation, thereby decreasing the transcriptional and mitogenic activity of RXR [249]. It appears that the interactions and effects of NR4A1/RXR heterodimerization are dependent on many factors, and that seemingly contradictory findings may be due to differences in cell types among other factors. There are only a few miRNAs that directly target $R X R A$, including miR-128 and miR-574 [250, 251]. Therefore it is possible that expression of these miRNAs result in the opposite effects described here.

COUP-TF. In addition to RXR, the COUP-TF (chicken ovalbumin upstream promoter-transcription factor) orphan receptors can also bind with NR4A1 and have varying effects. In the absence of ligand, COUP-TFs bind RAREs and inhibit their transcription, leading to decreased RARB and increased cell growth, at least in breast and lung cancer. However in the presence of RA, COUP-TF has no effect on $\beta$ RARE transcription and therefore enhances the anti-tumor effects of RA, leading to the conclusion that COUP-TF sensitizes cancer cells to RA. In lung cancer cells, NR4A1 heterodimerizes with COUP-TF and prevents binding to the $\beta$ RARE, thereby desensitizing the cells to RA. On the other hand, COUP-TF can prevent NR4A1/RXR heterodimer binding to the $\beta$ RARE via protein-protein interaction. Furthermore, COUP- 
Table 2-1. Effects of Nur77 regulatory networks on cancer and potential implications by miRNAs.

\begin{tabular}{|c|c|c|c|c|c|c|}
\hline $\begin{array}{l}\text { Functional } \\
\text { relationship to } \\
\text { NR4A }\end{array}$ & $\begin{array}{l}\text { Gene } \\
\text { (common } \\
\text { name) }\end{array}$ & $\begin{array}{l}\text { Effect on NR4A/functional } \\
\text { consequence }\end{array}$ & $\begin{array}{l}\text { Oncogenic } \\
\text { effect? }\end{array}$ & Cancer type/disease & miRNAs & $\begin{array}{l}\text { miRNA } \\
\text { references }\end{array}$ \\
\hline \multirow{5}{*}{$\begin{array}{l}\text { Modulates } \\
\text { Nur77 mRNA } \\
\text { expression }\end{array}$} & $\begin{array}{l}E P 300 \\
(\mathrm{p} 300)\end{array}$ & Promotes expression via acetylation & Yes & Liver, cervical & $\begin{array}{l}20 \mathrm{a}, 26 \mathrm{a}, 106 \mathrm{~b} \sim 25,132,150, \\
574\end{array}$ & {$[252-257]$} \\
\hline & $\begin{array}{l}\text { Fos/Jun } \\
\text { (AP-1) }\end{array}$ & $\begin{array}{l}\text { Promotes expression by directly } \\
\text { binding to promoter }\end{array}$ & Yes & Colon & $101,181 b, 155$ & {$[258-263]$} \\
\hline & $\begin{array}{l}H I F 1 A \\
(\mathrm{HIF}-1 \alpha)\end{array}$ & $\begin{array}{l}\text { Promotes expression by directly } \\
\text { binding to promoter }\end{array}$ & Yes & $\begin{array}{l}\text { Neuroblastoma, } \\
\text { cervical, breast, liver }\end{array}$ & $\begin{array}{l}17 \sim 92,18 \mathrm{a}, 20 \mathrm{~b}, 31,93,138, \\
155,199 \mathrm{ab}, 206,210,335, \\
338,519 \mathrm{c}\end{array}$ & [264-279] \\
\hline & $\begin{array}{l}C T N N B 1 \\
(\beta \text {-catenin })\end{array}$ & $\begin{array}{l}\text { Promotes expression via activation of } \\
\text { AP-1 }\end{array}$ & Yes & Colon & $\begin{array}{l}34,185,200 \mathrm{abc}, 203,214 \\
434,680,690\end{array}$ & {$[280-286]$} \\
\hline & $H D A C 1$ & Suppresses expression & No & Liver, cervical & $34 a, 449 a b, 874$ & {$[287-294]$} \\
\hline \multirow[t]{7}{*}{$\begin{array}{l}\text { Binds Nur77 } \\
\text { protein }\end{array}$} & $\begin{array}{l}R X R A \\
(\operatorname{RXR} \alpha)\end{array}$ & $\begin{array}{l}\text { RXR/Nur } 77 \text { heterodimer either } \\
\text { activates transcription or translocates } \\
\text { to mitochondria to induce apoptosis }\end{array}$ & $\begin{array}{l}\text { Yes and } \\
\text { No }\end{array}$ & Lung, pancreatic & 128,574 & {$[250,251]$} \\
\hline & $\begin{array}{l}N R 2 F 2 \\
(\mathrm{COUP}-\mathrm{TF})\end{array}$ & $\begin{array}{l}\text { Binds and prevents Nur } 77 \text { from } \\
\text { binding to RXR, thereby resensitizing } \\
\text { cells to RA }\end{array}$ & $\begin{array}{l}\text { Yes and } \\
\text { No }\end{array}$ & Lung & $194,302 a$ & {$[295,296]$} \\
\hline & CHDIL & Inhibits mitochondrial targeting & Yes & Liver & None & \\
\hline & $\begin{array}{l}X R C C 5 \\
(\mathrm{Ku} 80)\end{array}$ & $\begin{array}{l}\text { Nur77 prevents Ku80-mediated DNA } \\
\text { repair }\end{array}$ & No & Liver & $31,526 b$ & {$[297,298]$} \\
\hline & TP53 (p53) & $\begin{array}{l}\text { Nur } 77 \text { blocks p53 transcriptional } \\
\text { activity/promotes p53-mediated } \\
\text { apoptosis }\end{array}$ & $\begin{array}{l}\text { Yes and } \\
\text { No }\end{array}$ & Lung, liver, bone & $\begin{array}{l}25,30 \mathrm{~d}, 125,150,375,504 \\
1285\end{array}$ & {$[299-305]$} \\
\hline & $\begin{array}{l}\text { STK11 } \\
(\mathrm{LKB} 1)\end{array}$ & $\begin{array}{l}\text { Nur77 prevents LKB1 from } \\
\text { suppressing mTOR }\end{array}$ & Yes & Cervical & 155 & {$[306]$} \\
\hline & PIN1 (Pin1) & $\begin{array}{l}\text { Pin } 1 \text { promotes Nur } 77 \text { transcriptional } \\
\text { activity }\end{array}$ & Yes & Cervical & 140, 200bc, 296 & {$[307-309]$} \\
\hline
\end{tabular}


Table 2-1. Continued.

\begin{tabular}{|c|c|c|c|c|c|c|}
\hline \multirow[t]{8}{*}{$\begin{array}{l}\text { Functional } \\
\text { relationship to } \\
\text { NR4A }\end{array}$} & $\begin{array}{l}\text { Gene } \\
\text { (common } \\
\text { name) }\end{array}$ & $\begin{array}{l}\text { Effect on NR4A/functional } \\
\text { consequence }\end{array}$ & $\begin{array}{l}\text { Oncogenic } \\
\text { effect? }\end{array}$ & Cancer type/disease & miRNAs & $\begin{array}{l}\text { miRNA } \\
\text { references }\end{array}$ \\
\hline & $\begin{array}{l}E P 300 \\
(\mathrm{p} 300)\end{array}$ & $\begin{array}{l}\text { Nur77 either inhibits p300 or forms } \\
\text { complex to promote transcription }\end{array}$ & $\begin{array}{l}\text { Yes and } \\
\text { No }\end{array}$ & Cervical, breast, lung & $\begin{array}{l}20 \mathrm{a}, 26 \mathrm{a}, 106 \mathrm{~b} \sim 25,132,150, \\
574\end{array}$ & {$[252-257]$} \\
\hline & $\begin{array}{l}C T N N B 1 \\
(\beta \text {-catenin) }\end{array}$ & Nur77 inhibits $\beta$-catenin function & No & Colon & $\begin{array}{l}34,185,200 \mathrm{abc}, 203,214 \\
434,680,690\end{array}$ & {$[280-286]$} \\
\hline & $N D R G 1$ & $\begin{array}{l}\text { Competitively binds Nur77 and } \\
\text { prevents it from inducing } \beta \text {-catenin } \\
\text { degradation }\end{array}$ & Yes & Liver & 182,769 & {$[310,311]$} \\
\hline & $\begin{array}{l}V H L \\
(\mathrm{pVHL})\end{array}$ & $\begin{array}{l}\text { Nur77 inhibits the pVHL-mediated } \\
\text { ubiquitination of HIF-1 } \alpha\end{array}$ & Yes & $\begin{array}{l}\text { Neuroblastoma, renal } \\
\text { cell carcinoma }\end{array}$ & 21 & [312] \\
\hline & $\begin{array}{l}\text { NROB2 } \\
(\mathrm{SHP})\end{array}$ & $\begin{array}{l}\text { SHP blocks Nur77 from } \\
\text { transcriptionally mediating cell death } \\
\text { upon treatment with an apoptosis- } \\
\text { inducer }\end{array}$ & Yes & Liver & $141,378 \mathrm{~g}, 4649$ & {$[313-315]$} \\
\hline & $\begin{array}{l}P R K C A \\
(\mathrm{PKC})\end{array}$ & $\begin{array}{l}\text { Nur77 prevents PKC from activating } \\
\text { AP-1 and NF-kB }\end{array}$ & No & Acute $\mathrm{T}$ cell leukemia & $24-2$ & [316] \\
\hline & $P M L$ & Inhibits Nur77 transcriptional activity & No & osteosarcoma & None & \\
\hline \multirow[t]{3}{*}{$\begin{array}{l}\text { Phosphorylates } \\
\text { Nur77 }\end{array}$} & $A K T 1$ (Akt) & $\begin{array}{l}\text { Nur77 mitochondrial targeting is } \\
\text { blocked by Akt }\end{array}$ & Yes & Gastric & $100,105,133 b, 143,149,342$ & {$[317-322]$} \\
\hline & $J N K$ & $\begin{array}{l}\text { JNK induces Nur } 77 \text { mitochondrial } \\
\text { targeting and inhibits its transcriptional } \\
\text { activity }\end{array}$ & No & $\begin{array}{l}\text { Lung, prostate, breast, } \\
\text { glioma, ovarian, oral } \\
\text { squamous cell } \\
\text { carcinoma }\end{array}$ & $92 \mathrm{a}$ & {$[323]$} \\
\hline & $\begin{array}{l}\text { PRKDC } \\
\text { (DNA- } \\
\text { PKcs) }\end{array}$ & $\begin{array}{l}\text { Nur77 induces apoptosis upon ionizing } \\
\text { radiation treatment, which is dependent } \\
\text { on its phosphorylation by DNA-PKcs }\end{array}$ & No & Liver & 101 & {$[324]$} \\
\hline
\end{tabular}




\section{Table 2-1. Continued.}

\begin{tabular}{|c|c|c|c|c|c|c|}
\hline $\begin{array}{l}\text { Functional } \\
\text { relationship to } \\
\text { NR4A }\end{array}$ & $\begin{array}{l}\text { Gene } \\
\text { (common } \\
\text { name) }\end{array}$ & $\begin{array}{l}\text { Effect on NR4A/functional } \\
\text { consequence }\end{array}$ & $\begin{array}{l}\text { Oncogenic } \\
\text { effect? }\end{array}$ & Cancer type/disease & miRNAs & $\begin{array}{l}\text { miRNA } \\
\text { references }\end{array}$ \\
\hline & $\begin{array}{l}G S K 3 B \\
(G S K-3 \beta)\end{array}$ & $\begin{array}{l}\text { Nur77 is blocked from inhibiting B- } \\
\text { catenin transcriptional activity upon } \\
\text { phosphorylation by GSK-3B }\end{array}$ & Yes & Colon & $26 a, 346$ & {$[325,326]$} \\
\hline \multirow[t]{9}{*}{$\begin{array}{l}\text { Transcriptional } \\
\text { target of Nur77 }\end{array}$} & $\begin{array}{l}\text { CCND2 } \\
\text { (cyclin D2) }\end{array}$ & Promotes proliferation via cyclin D2 & Yes & Cervical & $\begin{array}{l}\text { 1, let-7a, 15b, 16, 26a, 29abc, } \\
\text { 30c, 98, 124a, 145, 182, 195, } \\
\text { 198, 204, 206, 302b, 340, 375, } \\
497,610\end{array}$ & {$[327-350]$} \\
\hline & $T X N D C 5$ & $\begin{array}{l}\text { Promotes lower stress levels and } \\
\text { therefore increased survival }\end{array}$ & Yes & Pancreatic & $200 \mathrm{~b}$ & {$[351]$} \\
\hline & $\begin{array}{l}\text { BIRC5 } \\
\text { (survivin) }\end{array}$ & Promotes survival & Yes & Pancreatic & $\begin{array}{l}16,195 \sim 497,203,218,542, \\
708\end{array}$ & {$[352-361]$} \\
\hline & $E 2 F 1$ & $\begin{array}{l}\text { TPA induces Nur } 77 \text {, resulting in } \\
\text { increased E2F1 and apoptosis; Pin1 } \\
\text { promotes Nur77 to transcriptionally } \\
\text { activate E2F1, resulting in increased } \\
\text { proliferation }\end{array}$ & $\begin{array}{l}\text { Yes and } \\
\text { No }\end{array}$ & Prostate, cervical & $\begin{array}{l}106 \mathrm{a}, 136,149,205,223,320, \\
326,329,330,331,342,362, \\
493,603\end{array}$ & $\begin{array}{l}{[321,362-} \\
374]\end{array}$ \\
\hline & $C D K 4$ & $\begin{array}{l}\text { Nur77 activates CDK4 following } \\
\text { induction by bile acids }\end{array}$ & Yes & Liver, colon & $\begin{array}{l}1,34 \mathrm{a}, 124,188,195,206 \\
486,506,613\end{array}$ & $\begin{array}{l}{[341,375-} \\
387]\end{array}$ \\
\hline & $B R E$ & $\begin{array}{l}\text { Nur77 activates BRE following } \\
\text { induction by bile acids }\end{array}$ & Yes & Liver, colon & None & \\
\hline & $R B B P 8$ & $\begin{array}{l}\text { Nur } 77 \text { activates RBBP8 following } \\
\text { induction by bile acids }\end{array}$ & Yes & Liver, colon & $19 \mathrm{ab}, 335$ & {$[388,389]$} \\
\hline & $M A P 4 K 5$ & $\begin{array}{l}\text { Nur77 activates MAP4K5 following } \\
\text { induction by bile acids }\end{array}$ & Yes & Liver, colon & None & \\
\hline & STAT5A & $\begin{array}{l}\text { Nur77 activates STAT5A following } \\
\text { induction by bile acids }\end{array}$ & Yes & Liver, colon & $141,222,223,1469$ & {$[390-393]$} \\
\hline
\end{tabular}


Table 2-1. Continued.

\begin{tabular}{|c|c|c|c|c|c|c|}
\hline $\begin{array}{l}\text { Functional } \\
\text { relationship to } \\
\text { NR4A }\end{array}$ & $\begin{array}{l}\text { Gene } \\
\text { (common } \\
\text { name) }\end{array}$ & $\begin{array}{l}\text { Effect on NR4A/functional } \\
\text { consequence }\end{array}$ & $\begin{array}{l}\text { Oncogenic } \\
\text { effect? }\end{array}$ & Cancer type/disease & miRNAs & $\begin{array}{l}\text { miRNA } \\
\text { references }\end{array}$ \\
\hline & $B I D$ & $\begin{array}{l}\text { Nur77 activates BID following } \\
\text { induction by bile acids }\end{array}$ & No & Liver, colon & None & \\
\hline & POMC & Nur77 promotes POMC expression & Yes & Cushing's disease & None & \\
\hline & CITED1 & $\begin{array}{l}\text { Nur77 represses CITED1, allowing } \\
\text { Wnt activation }\end{array}$ & Yes & Melanoma & None & \\
\hline & $D A C T 1$ & $\begin{array}{l}\text { Nur77 represses DACT1, allowing } \\
\text { Wnt activation }\end{array}$ & Yes & Melanoma & None & \\
\hline & $M Y C$ & Nur77 represses MYC & No & $\begin{array}{l}\text { Acute myeloid } \\
\text { leukemia }\end{array}$ & $\begin{array}{l}\text { let-7g, } 24,34 a b c, 126,135 b \text {, } \\
145,185,320 b, 487 b, 494 \text {, } \\
744\end{array}$ & {$[394-408]$} \\
\hline
\end{tabular}




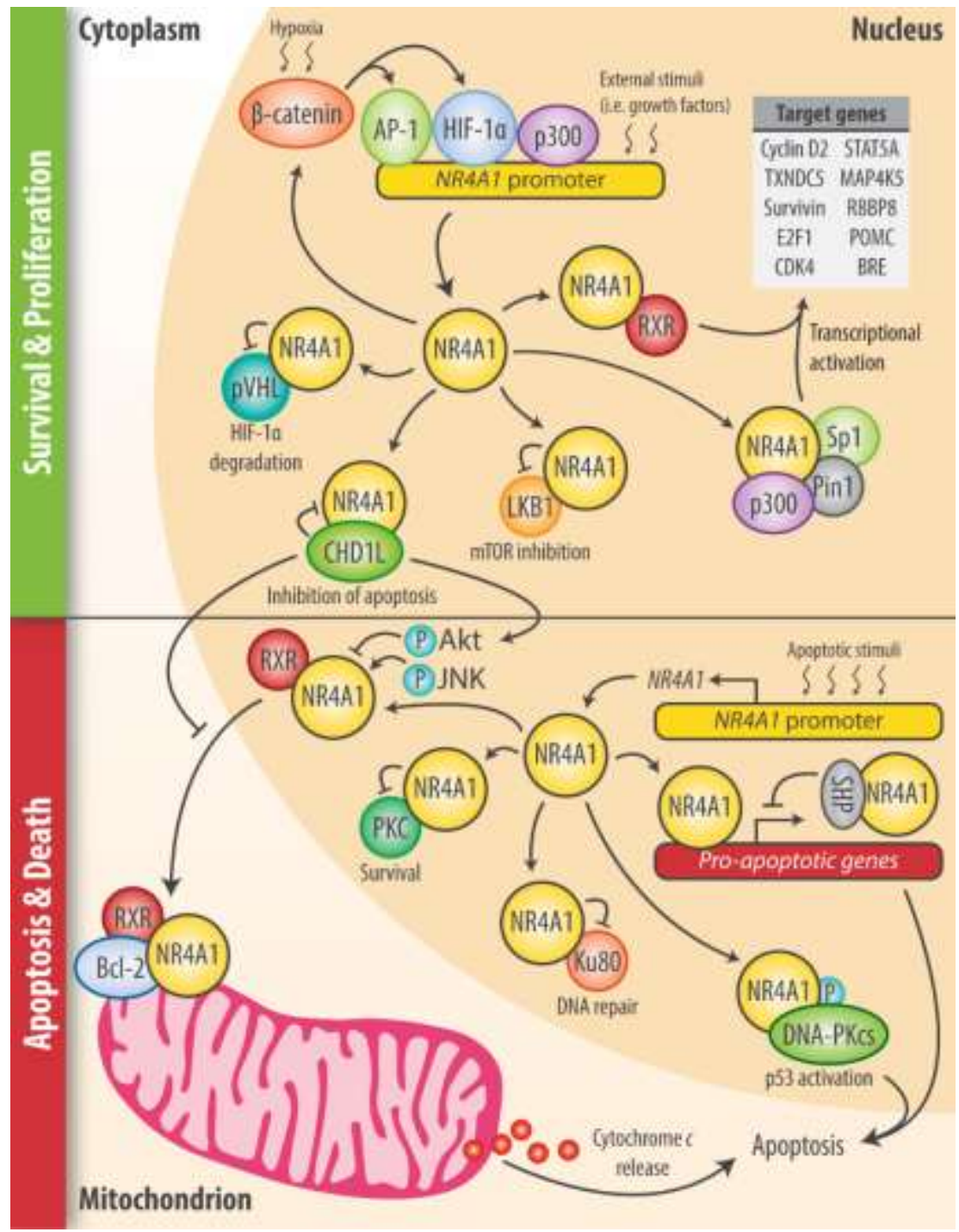

Figure 2-1. NR4A1 mediates cell proliferation and survival in addition to cell death.

The top panel depicts genes that transcriptionally activate $N R 4 A 1$, leading to NR4A1 interactions that ultimately promote cell proliferation and survival. In contrast, the bottom panel details the actions of NR4A1 in response to apoptotic stimuli, resulting in translocation of NR4A1 to the mitochondria and induction of apoptosis. 
TF expression was positively correlated with RA sensitivity, while NR4A1 expression was associated with retinoid resistance in lung cancer cells. This suggests NR4A1 is responsible for RA resistance, leading to enhanced cell proliferation [247]. miRNAs capable of directly binding and suppressing NR2F2 (encodes COUP-TF) include miR194 and miR-302a. COUP-TF suppresses osteoblastic differentiation, and overexpression of miR-194 and miR-302a was found to rescue this effect [295, 296]. This leads us to speculate that expression of these miRNAs would result in the desensitization of cancer cells to RA treatment mediated by NR4A1.

CHD1L. The next gene that physically interacts with NR4A1 is CHD1L. This protein acts as an oncogene in hepatocellular carcinoma and can bind with NR4A1 to prevent its translocation to the mitochondria and subsequent apoptosis upon treatment with staurosporine [409]. Unfortunately, there are no miRNAs that have been validated to target CHD1L. However, CHD1L interacts with Ku70 and DNA-dependent protein kinase, catalytic subunit (DNA-PKcs) to enhance chromatin remodeling and DNA repair [410, 411], although its elevated levels in cancer surprisingly lead to increased DNA damage. The DNA-PK complex is essential for mediating double-strand break repair and comprises DNA-PKcs and Ku70/Ku80. Since NR4A1 is known to interact with Ku80 to suppress DNA repair [232] and has also been shown to bind CHD1L, it is possible that NR4A1 inhibits DNA repair via protein-protein interactions with Ku80 and/or CHD1L. There are presently two miRNAs shown to target XRCC5 (encodes Ku80), including miR-31 and miR-526b [297, 298]. Therefore expression of these miRNAs may lead to increased DNA damage, which would most likely lead to cell death.

p53. One of the more significant binding partners of NR4A1 is the tumor suppressor $\mathrm{p} 53$, and similar to some of its previously discussed binding partners, this interaction can lead to contradictory outcomes. NR4A1 can play an oncogenic role by binding to $\mathrm{p} 53$ and preventing p300 acetylation of p53, resulting in suppressed p53 transcriptional activity [412]. In addition, NR4A1 can circuitously promote oncogenic mTOR signaling by inhibiting p53-mediated transcription of sestrin-2, an activator of AMPK, which is an inhibitor of mTOR $[238,413]$. However in the presence of DNAPKcs, NR4A1 is phosphorylated by DNA-PKcs and becomes an activator of p53 transcriptional activity via phosphorylation of $\mathrm{p} 53$ by DNA-PKcs. In addition to increasing p53 transactivation, NR4A1 heterodimerization with p53 can enhance p53mediated apoptosis as well as $\mathrm{p} 53$ protein stability by blocking ubiquitination and subsequent degradation of $\mathrm{p} 53$ by MDM2 [412]. As listed in Table 2-1, many miRNAs have been found to target TP53. Since p53 is a tumor suppressor, this would imply that any miRNA that targets it is likely an oncomiR.

LKB1. Another way in which NR4A1 promotes mTOR signaling is by inhibiting LKB1. NR4A1 binds LKB1 and blocks it from activating AMPK, thereby preventing AMPK from inhibiting mTOR. This leads to enhanced mTOR signaling and therefore increased proliferation, survival, and angiogenesis [414]. Interestingly, miR-155 was found to promote proliferation of cervical cancer cells by targeting STK11 (encodes LKB1), therefore acting as an oncomiR [306]. 
Pin1 and p300. Additional proteins that interact with NR4A1 include p300 and Pin1. NR4A1 isomerization and increased protein stability is mediated by Pin 1 and enhances recruitment of p300, thereby increasing the transcriptional activity of NR4A1 and its downstream proliferative effects [415]. NR4A1 can also form a complex with p300 and Sp1 to promote expression of proliferative and prosurvival genes such as survivin in lung cancer cells [238]. On the other hand, NR4A1 can directly interact with p300 and block its ability to acetylate transcription factors, thereby repressing their transcriptional activity and resulting in decreased proliferation in breast cancer cells [416]. Five miRNAs target EP300, including miR-20a [252], miR-132 [256], miR-150 [254], and miR-574 [253], as well as the miR-106b 25 cluster [255]. In addition, miR200b [307], miR-200c [308], and miR-296 [309] were found to target PIN1. All of these miRNAs, with the exception of miR-20a and the miR-106b 25 cluster, play mostly tumor suppressive roles. Therefore it would make sense that expression of these miRNAs would have a negative impact on NR4A1 function via targeting of EP300 and PIN1.

$\boldsymbol{\beta}$-catenin. The proto-oncogene $\beta$-catenin is often mutated in cancer and interacts with transcription factors to promote expression of mitogenic genes. NR4A1 acts as a negative regulator of $\beta$-catenin function either by inducing its degradation in the cytoplasm [417] or by blocking its interaction with the transcription factor TCF4 and promoting recruitment of corepressors, thereby suppressing its transcriptional activity in colon cancer [418]. In contrast, NR4A1 was found to activate and stabilize $\beta$-catenin protein and prevent its degradation under hypoxic conditions via NR4A1-mediated activation of the Akt pathway. As mentioned, $\beta$-catenin can promote NR4A1 expression, thereby forming a positive feedback loop that promotes the proliferation of colon cancer cells [244]. CTNNB1 (encodes $\beta$-catenin) is targeted by miR-34 [280, 419], miR-200a [282-284], and miR-214 [285, 286], all of which act as tumor suppressors in several cancer types. Five other miRNAs have also been shown to directly target CTNNB1 [281], and expression of these miRNAs may either relieve NR4A1 of its inhibitory effect on $\beta$ catenin, or suppress the NR4A1-mediated activation of $\beta$-catenin induced by hypoxia.

NDRG1. N-myc downstream regulated gene 1 (NDRG1) acts as an oncogene in hepatocellular carcinoma by promoting $\beta$-catenin accumulation. It does so by competitively binding with glycogen synthase kinase-3 3 (GSK-3 $\beta$ ) and NR4A1 individually, thus preventing $\beta$-catenin degradation by GSK-3 $\beta$ and NR4A1. This inhibitory mechanism by NDRG1 results in upregulation of downstream oncogenic genes [420]. miR-182 acts as an oncomiR in prostate cancer [310] while miR-769 [311] plays a tumor suppressive role in breast cancer, both of which mediate their effects by directly targeting $N D R G 1$. However in HCC, expression of these miRNAs would presumably result in increased $\beta$-catenin degradation via NR4A1 and GSK-3 $\beta$, thus leading to antiproliferative effects.

SHP. Small heterodimer partner (SHP) commonly inhibits nuclear receptor function via physical interaction and was found to bind and suppress NR4A1 transcriptional activity. When HCC cells were treated with an inducer of apoptosis, SHP prevented NR4A1 from transcriptionally mediating cell death [421]. There are three miRNAs that target NROB2 (encodes SHP), including miR-141 [313], miR-378g [314], 
and miR-4649 [315], expression of which would theoretically lead to enhanced apoptosis mediated by NR4A1.

PKC. As mentioned briefly, NR4A1 appears to be a tumor suppressor in leukemia. One way NR4A1 may mediate this effect is by interacting with and inhibiting protein kinase $\mathrm{C}$ (PKC). In Jurkat leukemic T cells, the LBD of NR4A1 binds PKC and prevents it from activating AP-1 and NF-kB [422]. The activation of NF-kB by PKC can lead to induction of anti-apoptotic genes, and since NR4A1 is known to induce apoptosis in T cells, its inhibition on PKC may be necessary in order for apoptosis to occur. miR24-2 is the only miRNA that has been shown to directly target PRKCA (PKC) and was found to decrease cell survival in breast cancer cells via PKC inhibition [316].

PML. Another way NR4A1 is involved in leukemia is through its interaction with the promyelocytic leukemia gene, PML [423]. Patients with acute promyelocytic leukemia commonly have a chromosomal translocation that results in fusion genes encoding PML-RAR $\alpha$ and RAR $\alpha$-PML [424]. PML is well known for its ability to inhibit cell proliferation and to act as a tumor suppressor in vivo [425-429]. PML was also found

to directly interact with NR4A1 in its DBD domain and prevent its transcriptional activity in osteosarcoma cells [423]. Thus far there have not been any miRNAs found to target $P M L$, but hypothetically any miRNA that suppresses PML would result in increased transcriptional activity by NR4A1.

VHL. Lastly, the tumor suppressor von Hippel-Lindau (pVHL) is an E3 ubiquitin ligase that forms a multimeric complex to degrade proteins and has been implicated in several diseases as a result of mutations in pVHL. One of its target proteins is HIF-1 $\alpha$, which gets ubiquitinated upon binding with pVHL and subsequently degraded. However, NR4A1 is able to bind pVHL and prevent this HIF-1 $\alpha$ ubiquination [430]. As mentioned, HIF-1 $\alpha$ can transcriptionally promote expression of NR4A1, which was shown in VHLdeficient renal cell carcinoma [245], as well as other cancer cell lines [246]. Since both NR4A1 and HIF-1 $\alpha$ are known to enhance expression of oncogenic genes via their transactivation functions, it is understandable that they would have a synergistic effect. $V H L$ is targeted by miR-21 [312], expression of which would result in increased HIF-1 $\alpha$ protein stability and transcriptional activity.

\section{Proteins that phosphorylate NR4A1}

Akt. Furthermore, miRNAs may target protein kinases that are responsible for the copious amounts of phosphorylation endured by NR4A1 on its N-terminus [86]. Some of these kinases positively regulate NR4A1 and mediate either its cell death or cell proliferation effects, while other kinases block these functions. Most studies that involve NR4A1-mediated apoptosis use external stimuli to force these effects. For example, gastric cancer cells treated with TPA experience cell death due to the translocation of NR4A1 to the mitochondria and ensuing induction of apoptosis. However, phosphorylation of NR4A1 by Akt, a serine/threonine kinase, blocks the mitochondrial targeting of NR4A1 along with its interaction with Bcl-2 [431]. Other studies have also 
verified this negative effect of Akt on NR4A1-induced apoptosis, as well as an inhibitory effect on NR4A1 transcriptional activity [121, 432]. Furthermore, CHD1L indirectly activates Akt, leading to inhibition of cell death [433]. As discussed, CHD1L is also able to block apoptosis by directly binding NR4A1; therefore the activation of Akt is an additional mechanism by which CHD1L can prevent cell death via inhibition of NR4A1. miRNAs that target $A K T 1$ would theoretically alleviate the suppression of NR4A1 by Akt. Several of these miRNAs act as tumor suppressors, including miR-105 and miR$133 \mathrm{~b}$, which were found to act as tumor suppressors in HCC and bladder cancer, respectively, via inhibition of $A K T 1$ [318, 319].

JNK. On the other hand, phosphorylation of NR4A1 by c-Jun N terminal kinase (JNK) promotes the translocation and mitochondrial targeting of NR4A1. This NR4A1mediated apoptosis occurs in the presence of several different cytotoxic compounds. Many of these compounds also upregulate NR4A1 expression, including 6-[3-(1adamantyl)-4-hydroxyphenyl]- 2-naphthalene carboxylic acid (AHPN). This retinoid is a potent inducer of apoptosis in several cancer types, and addition of 3-Cl-AHPC (an analog of AHPN) to lung, prostate, and breast cancer cells induces phosphorylation of NR4A1 by JNK followed by its translocation to the mitochondria and subsequent apoptosis [167, 434]. Treatment of lung cancer cells with 3-Cl-AHPC also inhibits NR4A1 transcriptional activity [167]. In addition, apaensin, a plant-derived natural product, induces apoptosis in lung and breast cancer cells via NR4A1, which is mediated by JNK phosphorylation [435]. Furthermore, glioma and oral squamous cell carcinoma treated with PCH4, a derivative of n-butylidenephthalide, induced apoptosis, which was dependent upon the mitochondrial targeting of NR4A1 mediated by JNK $[436,437]$. Interestingly, the nuclear export of NR4A1 occurs in cisplatin-sensitive ovarian cancer cells but not in cisplatin-resistant cells. Cisplatin-induced apoptosis is dependent on the translocation of NR4A1 mediated by JNK phosphorylation [179]. There are no known miRNAs that target human MAPK8 (JNK), although miR-92a has been shown to target mouse Mapk8 [323]. Expression of miR-92a would hypothetically suppress NR4A1 translocation and instead allow for cell survival and proliferation via inhibition of JNK.

DNA-PKes. In addition to JNK, DNA-PKcs also positively regulates NR4A1. DNA-PKcs phosphorylates NR4A1 and increases its protein levels in liver cancer cells. As mentioned previously, this phosphorylation of NR4A1 enhances the transcriptional activity and phosphorylation of p53 by DNA-PK. Hepatoma cells treated with ionizing radiation (IR) leads to DNA-PKcs-mediated upregulation of NR4A1, which results in apoptosis induced by NR4A1 [232]. miR-101 was found to sensitize glioma and lung tumors to radiation via direct suppression of PRKDC (encodes DNA-PKcs) [324]. Paradoxically, decreased $P R K D C$ by miR-101 should result in reduced NR4A1-mediated apoptosis, but perhaps miR-101-mediated apoptosis is occurring via NR4A1-independent mechanisms.

GSK-3及. As stated earlier, NR4A1 prohibits the transcriptional activity of $\beta$ catenin by disrupting the DNA binding of $\beta$-catenin and TCF4 and promotes the recruitment of corepressors to Wnt target genes, thereby attenuating tumor growth. However, this negative regulation by NR4A1 is prevented upon phosphorylation by 
GSK-3 $\beta$, which is a phenomenon observed in most clinical colorectal cancers [418]. This phosphorylation of NR4A1 by GSK-3 $\beta$ allows $\beta$-catenin to transcriptionally activate genes involved in cell proliferation, leading to cancer progression. This may seem contradictory to the main function of GSK-3 $\beta$, which is to promote degradation of $\beta$ catenin, leading to tumor suppression. However in colorectal cancer, GSK-3 $\beta$ may have evolved in the tumor microenvironment to instead support $\beta$-catenin activity via inhibition of NR4A1. Therefore in this scenario, miRNAs that target and inhibit GSK3B should lead to decreased tumor growth since GSK-3 $\beta$ can no longer prevent NR4A1mediated degradation of $\beta$-catenin. miR-26a promotes cholangiocarcinoma [325] and miR-346 promotes osteogenic differentiation [326], both of which mediate their effects by directly targeting $G S K 3 B$.

\section{Target genes of NR4A1}

Proliferation and survival genes. Lastly, miRNAs may attenuate the transcriptional activity of NR4A1 by targeting and suppressing the expression of NR4A1 target genes. Transcriptional targets of NR4A1 that mediate its proliferation and survival functions include CCND2 (cyclin D2) [415], E2F1 [415, 438], thioredoxin domain containing 5 (TXNDC5) [439], and BIRC5 (survivin) [233, 238]. Many of these genes act as oncogenes, for example survivin is overexpressed in many cancers including pancreatic cancer, where it was found that NR4A1-induced survivin expression is essential for pancreatic cancer cell growth [233, 238]. In addition to survivin, TXNDC5 is also upregulated in several cancer types and was found to promote proliferation and migration while decreasing apoptosis in gastric cancer cells [440]. TXNDC5 is induced by NR4A1 in pancreatic cancer cells, leading to lower stress levels that permit increased cancer cell growth and survival [439]. Cyclin D2 and E2F1 expression induced by NR4A1 was also found to increase cell proliferation [415], although induction of NR4A1 by TPA actually resulted in increased apoptosis via E2F1 in prostate cancer cells [438]. Numerous miRNAs have been found to directly target these genes, and expression of these miRNAs typically results in decreased proliferation and survival (Table 2-1).

Pro-inflammatory genes. Furthermore, liver and colon cancer cells treated with bile acids (BAs) experienced a substantial increase in NR4A1 expression, leading to the upregulation of pro-inflammatory genes subsequently identified as NR4A1 target genes. These include $C D K 4, C C N D 2, B R E, R B B P 8, M A P 4 K 5$, and STAT5A, which most likely mediate the increased proliferation and migration observed upon BA-induced NR4A1 overexpression [231]. Surprisingly, NR4A1 was also found to induce the proapoptotic gene $B I D$ [231]. miRNAs that target and suppress the expression of NR4A1 target genes would therefore attenuate the effects of NR4A1 transcriptional activity, and in this case the miRNAs would act as tumor suppressors in liver and colon cancers (Table 2-1).

POMC. An RXR agonist, HX630, suppresses NR4A1 and its transcriptional induction of pro-opiomelanocortin $(P O M C)$, leading to the inhibition of corticotroph tumor growth [441]. Corticotroph tumors that secrete excessive amounts of adrenocorticotropic hormone (ACTH) can develop into Cushing's disease [441]. 
Therefore, NR4A1-mediated induction of POMC may lead to the formation of corticotroph tumors and downstream Cushing's disease. miRNAs that block expression of POMC would thus decrease the formation of corticotroph tumors and the likelihood of Cushing's disease. Unfortunately, no miRNAs have been verified to target $P O M C$ as of yet.

Negative regulators of Wnt. Over 70\% of melanomas have a mutation in NRAS or BRAF, resulting in aberrant and constitutive signaling of the MAPK pathway and downstream proliferative effects. When NR4A1 is knocked down in melanoma cells via siRNA, antagonists of the Wnt pathway, DACT1 and CITED1, were upregulated [442]. Therefore, NR4A1 acts to transcriptionally suppress these antagonists, resulting in the continued activation of the Wnt pathway. This further supports the role of NR4A1 as an oncogenic factor, especially in the case of melanoma since NR4A1 has the highest expression in this cancer compared to all other cancer types. Currently, there are no known miRNAs that target $D A C T 1$ and $C I T E D 1$, although several miRNAs are predicted to target DACT1 in the TargetScan database. Expression of these predicted miRNAs would theoretically support the function of NR4A1 and promote activation of the Wnt pathway.

Myc and Bcl-2. On the other hand, NR4A1 plays a tumor suppressive role in AML by binding to the promoter of $M Y C$ and suppressing its transcription, thereby preventing its downstream oncogenic effects. NR4A1 was also found to decrease BCL2 expression, although it is unclear if it directly binds the promoter. Myc and Bcl-2 work together to promote AML, therefore NR4A1-mediated suppression of BCL2 further attenuates the oncogenic activity of Myc. Furthermore, the leukemogenicity of AML cells was prevented by overexpression of NR4A1 in vivo [443]. There are many miRNAs that target $M Y C$ and $B C L 2$, and expression of these miRNAs would support the role of NR4A1 as a tumor suppressor in AML.

\section{Conclusions}

The regulatory proteins listed in this chapter are not an exhaustive list of those that regulate and interact with NR4A1. There are many others that target it in different contexts, however only the proteins that have been shown to target and regulate NR4A1 in the context of cancer are discussed. A more thorough review that was recently published includes all interacting factors of NR4A1 and its family members and explores how these interactions affect various physiological processes [444].

In addition to the proteins discussed here, other transcription factors such as Sp1, NF-kB, and CREB have all been shown to bind the promoter of NR4A1 to modulate its expression [137, 445, 446]. Other binding partners also include NFAT, GATA4, SRC-2, and c-JUN [77, 447-449]. An important binding partner of NR4A1 that mediates the apoptotic effect of NR4A1 is Bcl-2 [123]. Additional kinases such as ERK2, ERK5, RSK2, and p38 $\alpha$ also phosphorylate NR4A1 [450-453]. Furthermore, NR4A1 has been shown to bind the promoters and modulate expression of EDN1 (endothelin 1) and 
ITGB4 (integrin beta 4) [448, 454]. However, these proteins were not discussed in detail since the experiments were performed in relatively normal cell lines. Due to the fact that NR4A1 may play different and opposing roles depending on the cell line, it is possible that it may also have different functions in cancer cells compared to normal cells. Because NR4A1 appears to play an oncogenic role in cancer, this chapter discusses the proteins that regulate NR4A1 in a malignant environment.

Many of the miRNAs discussed here correlate well with the role of NR4A1 in cancer. Those miRNAs that are typically overexpressed in cancer and act as oncomiRs appear to support the oncogenic functions of NR4A1 by suppressing proteins that would otherwise attenuate the actions of NR4A1 or repress oncogenic pathways. On the other hand, many of the miRNAs that target genes involved in NR4A1-mediated cancer progression are known tumor suppressors. All of the miRNAs discussed and listed in Table 2-1 have been verified to target their aforementioned targets using luciferase assays in which the seed region was mutated to confirm direct binding, and most of them were further verified by qPCR and western blot analysis.

It is important to note that in instances where NR4A1 mediates apoptosis in cancer cells, it is being induced to do so upon treatment with cytotoxic compounds. Therefore in the absence of external stimuli, it seems that the normal role of NR4A1 in cancer is to transcriptionally promote expression of genes that are important in cell proliferation and survival.

According to the latest research, there are numerous studies portraying NR4A1 as an oncogenic factor. Glancing at Table 2-1, it is apparent that NR4A1 exerts is mitogenic effects via several mechanisms. These include transcriptional regulation by other transcription factors, protein-protein interactions, post-translational modifications such as phosphorylation, and directly binding the promoters of target genes to drive their expression towards a proliferative and pro-survival state. Understanding these regulatory networks is imperative in order to develop more efficacious and personalized treatment for cancer patients. 


\section{CHAPTER 3. REGULATION OF NUCLEAR RECEPTOR NR4A1 BY MIR-124*}

\section{Introduction}

One of the ten hallmarks of cancer is uncontrolled and limitless cell growth. Cells that have acquired the capability to proliferate indefinitely form tumors that may metastasize and develop into cancer. In some cancers, this proliferative signaling is controlled by certain genes that are often overexpressed and thereby constitute ideal therapeutic targets. Some of these target genes include nuclear receptors such as the estrogen receptor in breast cancer or the androgen receptor in prostate cancer. Another nuclear receptor that researchers have identified as a potential therapeutic target is NR4A1.

The nuclear receptor NR4A1 is commonly upregulated in adult cancers and has oncogenic functions. NR4A1 is an immediate-early response gene that acts as a transcription factor to promote proliferation and protect cells from apoptosis. Conversely, NR4A1 can translocate to the mitochondria and induce apoptosis upon treatment with various cytotoxic agents. The roles of NR4A1 in cancers have been investigated mostly in adult cancers, with very few studies in childhood malignancies. NR4A1 is downregulated in leukemia, and $\mathrm{Nr} 4 a 1 / \mathrm{Nr} 4 \mathrm{a} 3$ double-knockout mice quickly develop acute myeloid leukemia before succumbing to the disease [156]; however, the expression and function of NR4A1 have not been well studied in pediatric solid tumors. Recent data from the Pediatric Cancer Genome Project show that NR4A1 is deleted in many hypodiploid acute lymphoblastic leukemia tumors, whereas it is amplified in some patients with Group 4 medulloblastoma (MB) and rhabdomyosarcoma [455].

Because NR4A1 is upregulated in cancer and may have a role in cancer progression, it is of interest to understand the mechanism controlling its expression. MicroRNAs (miRNAs) are responsible for inhibiting translation of their target genes by binding to the 3'UTR and either degrading the mRNA or preventing it from being translated into protein, thereby making these non-coding endogenous RNAs vital regulators of every cellular process. Several miRNAs have been predicted to target $N R 4 A 1$; however, strong evidence showing the regulation of $N R 4 A 1$ by any miRNA is lacking. In this study, we used a luciferase reporter assay containing the 3'UTR of NR4A1 to screen 296 miRNAs and found that miR-124, which is the most abundant miRNA in the brain and has a role in promoting neuronal differentiation, caused the greatest reduction in luciferase activity. Interestingly, we discovered an inverse relationship in Daoy medulloblastoma cells and undifferentiated granule neuron precursors in which $N R 4 A 1$ is upregulated and miR-124 is downregulated. Exogenous expression to further

* Modified with permission from PLOS ONE. Tenga, A., et al., Regulation of Nuclear Receptor Nur77 by miR-124. PLOS ONE, 2016. 11(2): p. e0148433. 
elevate NR4A1 levels in Daoy cells increased proliferation and viability, but knocking down NR4A1 via siRNA resulted in the opposite phenotype. Importantly, exogenous expression of miR-124 reduced NR4A1 expression, cell viability, proliferation, and tumor spheroid size in $3 \mathrm{D}$ culture. In all, we have discovered miR-124 to be downregulated in instances of medulloblastoma in which NR4A1 is upregulated, resulting in a proliferative state that abets cancer progression. This study provides evidence for increasing miR-124 expression as a potential therapy for cancers with elevated levels of NR4A1.

\section{Methods}

\section{Cell culture}

Human embryonic kidney cell line 293T (ATCC CRL-3216), human cortical neuronal cell line HCN-2 (ATCC CRL-10742), human medulloblastoma cell lines D341 (ATCC HTB-187) and Daoy (ATCC HTB-186), and human rhabdomyosarcoma cell lines RD (ATCC CCL-136) and SJCRh30 (ATCC CRL-2061) were obtained from ATCC (Manassas, VA). Cells were free from contamination of mycoplasma, and passaged for fewer than 6 months after receipt (or resuscitation). Human rhabdomyosarcoma cell line Rh41 has been described previously [456]. 293T, HCN-2, and RD cells were grown in Dulbecco's Modified Eagle's Medium (DMEM). Rh41 and Rh30 cells were grown in RPMI-1640 Medium, and Daoy cells were cultured in Eagle's Minimum Essential Medium (EMEM). UKF-NB-3 (NB3) cells, which originated from a patient with MYCNamplified stage 4 neuroblastoma [457], were cultured in Iscove's Modified Dulbecco's medium (IMDM). LHCN-M2 cells (immortalized myoblasts derived from the pectoralis major muscle [458]) were plated in gelatin-coated plates $(0.1 \%$ gelatin in PBS) and cultured in DMEM supplemented with 15\% FBS, 0.02M HEPES, $0.03 \mu \mathrm{g} / \mathrm{mL}$ zinc sulfate, $1.4 \mu \mathrm{g} / \mathrm{mL}$ vitamin B12, $0.055 \mu \mathrm{g} / \mathrm{mL}$ dexamethasone, $2.5 \mathrm{ng} / \mathrm{mL}$ hepatocyte growth factor (recombinant human), and $10 \mathrm{ng} / \mathrm{mL}$ basic fibroblast growth factor. All cells were cultured at $37^{\circ} \mathrm{C}$ in $5 \% \mathrm{CO} 2$, and all media (except LHCN-M2 media) were supplemented with 10\% fetal bovine serum, 1\% GlutaMAX, 1\% sodium pyruvate, and $1 \%$ penicillin-streptomycin (Life Technologies, Carlsbad, CA).

\section{Reporter assay}

For the miRNA screen, all miRNA constructs were obtained from an existing library [459]. These constructs are plasmids containing the pre-miRNA sequences and have been reconstituted in TE (10 mM Tris-HCl, $\mathrm{pH}$ 8.0; 0.1 mM EDTA, pH 8.0) buffer. Each miRNA construct $(0.09 \mu \mathrm{g})$ was first added to the well of a 96 -well plate (PerkinElmer, Waltham, MA) that were kept at $4^{\circ} \mathrm{C}$ until all miRNAs were plated. Next, $0.15 \mu \mathrm{g}$ of NR4A1-3'UTR reporter plasmid (NR4A1-3'UTR-Luc; GeneCopoeia, Rockville, MD) and $3 \mu \mathrm{L} / \mu \mathrm{g}$ of FuGENE 6 (Promega, Madison, WI) were mixed with 50 $\mu \mathrm{L}$ of Opti-MEM reduced-serum media (Life Technologies) and dispensed into each well 
before being overlaid with $293 \mathrm{~T}$ cells $(20,000$ cells/well in $50 \mu \mathrm{L}$ of antibiotic-free media). NR4A1-3'UTR-Luc (in the pEZX-MT01 vector) contains both the firefly luciferase gene (FLuc) fused upstream of the 3'UTR of NR4A1 under the control of the SV40 promoter and the Renilla luciferase gene ( $R L u c)$ under the control of the CMV promoter. RLuc was used as an internal transfection control. After 48 hours, the Dual-Glo luciferase assay system (Promega) was used to detect luciferase activity according to the manufacturer's instructions. Raw luciferase activity was measured by using the EnVision 2101 Multilabel Plate Reader (PerkinElmer). Raw values were normalized by dividing the FLuc values by the RLuc values and then normalized to the value of either pSIF, an empty vector control for miRNA that contains a scrambled sequence in place of the premiRNA sequence [459], or oligo control (Cntrl) in the miR-124 inhibitor assay. The $\mathrm{F} L u c / \mathrm{R} L u c$ values for pSIF or Cntrl were set as 1. Mutations in the 3'UTR of NR4A1 that disrupt the binding site of the miRNAs were made by Mutagenex (Suwanee, GA).

\section{RNA isolation and quantitative real-time PCR}

Total RNA, including miRNA, was extracted by using the Qiagen miRNeasy kit (Qiagen, Venlo, Netherlands); the Maxwell 16 LEV simplyRNA Tissue Kit was used with the Maxwell 16 Research Instrument (Promega) for RNA extraction only when miRNA extraction was not needed. RNA was converted to cDNA by using the SuperScript VILO cDNA Synthesis Kit (Life Technologies), and $2 \mu \mathrm{L}$ of $5 \mathrm{X}$ Taqman probes (Applied Biosystems) specific to each miRNA were added to enhance miRNA detection. Target gene mRNA expression was detected by using specific Taqman probes (20X) and quantitated via the 7900HT Fast Real-Time PCR System (Applied Biosystems). GAPDH (4352934E) was used as an endogenous control for all gene expression analysis, including NR4A1 (Assay ID Hs00374226_m1), E2F1 (Assay ID Hs00153451_m1), CCND2 (Assay ID Hs00153380_m1), BIRC 5 (Assay ID Hs04194392_s1), TXNDC5 (Assay ID Hs01046709_mH), CDK4 (Assay ID Hs01565683_g1), and STAT5A (Assay ID Hs00234181_m1). Both RNU6B (Assay ID 001093) and RNU48 (Assay ID 001006) were used as endogenous controls for miRNA expression. RNU48 was used instead of RNU6B to analyze endogenous miRNA expression in the cell lines because RNU48 had less variable $\mathrm{Ct}$ values among cell lines. The probes used to detect miRNA levels (Applied Biosystems) were miR-124-3p (Assay ID 001182), miR-15a-5p (Assay ID 000389), and miR-224-5p (Assay ID 002099).

Gapdh (4352932E) and Nr4al (Assay ID Mm01300401_m1) mouse probes were used to detect Gapdh and Nr4al expression in mice. snoRNA202 (Assay ID 001232) was used as an endogenous control for miR-124 expression in mice as recommended by Applied Biosystems [447]. The fold-change in expression was calculated by using the comparative $\mathrm{Ct}(\Delta \Delta \mathrm{Ct})$ method, with the values of controls set to 1. All samples were tested in quadruplicate. The Cancer miRNAs Transcriptome PCR Array (SABiosciences, MD) was used to identify potential miRNAs that target $N R 4 A 1$. The array was provided by the manufacturer in a 96-well PCR plate, each well containing cDNA sample synthesized from HeLa cells treated with one of 90 cancer-related miRNA mimics. According to the manufacturer's instruction, we added qPCR MasterMix and $N R 4 A 1$ probe and performed qPCR. We used the data analysis software provided by 
SABiosciences to analyze qPCR data and determine which miRNAs affect NR4A1 expression.

\section{miR-124 inhibitor assay}

Daoy cells were plated at a density of 100,000 cells per well in 6-well BD Falcon plates (Corning, Corning, NY). After 24 hours, the cells were first transfected with various concentrations of the miR-124-3p inhibitor (single-stranded RNA molecule) or the control oligonucleotide (oligo) for 24 hours at $37^{\circ} \mathrm{C}$ and then transfected with $1 \mu \mathrm{g}$ of the NR4A1-3'UTR-Luc reporter plasmid. Transfecting the miR-124-3p inhibitor first allowed it to sufficiently inhibit the miR-124 activity before transfection of the NR4AI3'UTR-Luc reporter plasmid. After 24 hours of incubation, the cells were reseeded at a density of 4,000 cells per well in a 96-well plate, with 6 wells used for each condition. The DualGlo reporter assay was performed 48 hours later. The mirVana inhibitor from Life Technologies and the Power inhibitor from Exiqon (Woburn, MA) were both used to obtain the inhibitor data as indicated in the figure legends; each was used with a control oligo from their respective manufacturers. The Lipofectamine RNAiMAX transfection reagent (Life Technologies) was used with both inhibitors.

\section{Transfections}

Cells were transfected with miRNAs by using Fugene6 (Promega) in Opti-MEM in combination with antibiotic-free media (corresponding to the cells being transfected). The plasmid containing pre-miR-124-1 and its backbone vector pEZX-MR03 were purchased from GeneCopoeia.

For the NR4A1 and miR-124 co-transfection assay, Daoy cells were seeded at a density of 100,000 cells per well in a 6-well BD Falcon plate. After 24 hours, cells were transfected with $1 \mu \mathrm{g}$ NR4A1 with or without its 3'UTR. Cells were transfected with 2.5 $\mu \mathrm{g}$ miR-124 or its control vector (MR03) 24 hours later and collected for Western blot analysis 48 hours after that.

For NR4A1-knockdown assays, Daoy cells were seeded at a density of 250,000 cells in T25 flasks. Once the cells were $60 \%-70 \%$ confluent, they were transfected with $20 \mathrm{nM}$ siNR4A1 by using Dharmacon siGENOME siRNA (GE Healthcare, Lafayette, $\mathrm{CO}$ ) and $8 \mu \mathrm{L}$ of RNAiMAX. After 48 hours, cells were reseeded for viability and proliferation assays. The SMARTpool siNR4A1 (Catalog \# M-003426-04) and the individual siNR4A1 (Catalog \# D-003426-23) were both used as indicated in the figure legends. Non-targeting siRNA \#4 (Catalog \# D-001210-04-20) was used as a control for both the pooled and individual siRNAs. 


\section{Molecular cloning}

NR4A1 cDNA was cloned into the pEXM12-3XFLAG (N-terminal) vector (GeneCopoeia). Forward (5'- ATACTAGTCCACCATGGACTACAAAGACC -3') and reverse (5'- ATG AAT TCC TAG AAG GGC AGC GTG TC -3') primers were used to PCR-amplify 3XFLAG-NR4A1 cDNA from the pEXM12-3XFLAG-NR4A1 vector. The NR4A1 PCR product was then cloned into the pCR2.1 TOPO vector by using the TOPO TA Cloning Kit (Life Technologies). The product was then digested by using SpeI and EcoRI restriction enzymes (New England BioLabs, Ipswich, MA) and ligated into a pSIN-EF2-IRES-Blast lentiviral expression vector to generate pSIN-NR4A1. The pSIN vector originated from Addgene (Plasmid \#16578, [460]) but was modified by inserting additional enzyme sites and the BlastR gene (for resistance to blasticidin).

To generate a pSIN-NR4A1-3'UTR construct, the 3'UTR of NR4A1 was cloned from the NR4A1-3'UTR reporter plasmid (GeneCopoeia) and inserted into the 3'UTR region downstream of the $N R 4 A 1$ coding sequence in the pSIN-NR4A1 vector. Briefly, the 3'UTR sequence was amplified by using forward $\left(5^{\prime}-\right.$ ATGAATTCCCCCTGCCTGGGAA $\left.-3^{\prime}\right)$ and reverse $\left(5^{\prime}-\right.$ ATGGATCCTTTTTTTTTTTTTTTTTTTTTTTTTTTTTTTCCAACTACATGT -3') primers. This 3'UTR PCR insert was electrophoresed on a gel, and the band was gelpurified by using the Qiagen gel extraction kit. The purified 3'UTR insert was then cloned into the pCR2.1 TOPO vector (Life Technologies). The TOPO and pSIN plasmids were digested by using EcoRI and BamHI (New England BioLabs), and the 3'UTR segment was ligated into the pSIN-NR4A1 vector. All primers were synthesized by Invitrogen; all PCR amplifications were performed by using the Phusion High-Fidelity PCR Master Mix with HF Buffer (New England BioLabs); and the sequences of all final DNA constructs were confirmed by performing Sanger sequencing.

\section{Protein isolation and Western blot analysis}

Cells were incubated with Pierce RIPA lysis buffer (Thermo Fisher Scientific, Grand Island, NY) on ice for 20 minutes and centrifuged at 17,500 g for 20 minutes. The supernatant was collected, and its protein concentration was measured by using a Pierce BCA Protein Assay kit. Absorbance at $540 \mathrm{nM}$ was measured by using the SpectraMax M5 microplate reader (Molecular Devices, Sunnyvale, CA). The protein was mixed with 10X loading buffer and 4X LDS (Life Technologies), incubated at $95^{\circ} \mathrm{C}$ for 5 minutes, and loaded into a NuPAGE 4-12\% Bis-Tris gel (Life Technologies). The separated protein was then transferred to a nitrocellulose membrane by using an iBlot transfer system (Invitrogen). The blot was blocked at room temperature for one hour by using Odyssey blocking buffer (LI-COR Biosciences, Lincoln, NE). Mouse monoclonal antiFlag M2 (Sigma; catalog \# F1804-5MG; used at 1:1500 dilution) and mouse monoclonal anti- $\beta$-actin (Sigma; A5441; used at 1:2000 dilution) antibodies were added and incubated overnight at $4^{\circ} \mathrm{C}$. After the primary antibodies were removed, the blot was washed three times with TBST for 15 minutes each time before being incubated with the secondary antibody for 1 hour at room temperature. After 1 hour, the blot was washed 
three times, and proteins were detected by using the Odyssey imaging system (LI-COR Biosciences). ImageJ [461] was used to measure band intensity.

\section{Lentivirus production and transduction}

Two million 293T cells were seeded into each $10-\mathrm{cm}$ dish. Once the cells reached approximately $90 \%$ confluence, they were transfected with $12 \mu \mathrm{g}$ of the expression plasmid, $3 \mu \mathrm{g}$ of the VSV-G envelope-expressing plasmid pMD2.G (Addgene, Plasmid \#12259), and $6 \mu \mathrm{g}$ of the 2nd-generation lentiviral packaging plasmid psPAX2 (Addgene, Plasmid \#12260) by using $60 \mu \mathrm{L}$ FuGENE6 (Promega) in OptiMEM. Media were replaced with fresh media 24 hours after transfection. The lentivirus supernatant was collected 48 hours after media replacement and filtered through a $0.45-\mu \mathrm{M}$ filter and titrated by using Lenti-X GoStix (Clontech Laboratories, Mountain View, CA). In cases of low titer, Lenti-X Concentrator (Clontech Laboratories) was used to increase the lentiviral titer.

To exogenously express NR4A1, Daoy cells were seeded at a density of 250,000 cells in T25 flasks. Once the cells were $60 \%-70 \%$ confluent, they were transduced with NR4A1 lentivirus; Polybrene (AmericanBio, Natick, MA) was used at $0.8 \mu \mathrm{L} / \mathrm{mL}$ to aid transduction efficiency. After 48 hours, cells were reseeded for viability and proliferation experiments.

\section{Viability assay}

Cells were reseeded at a density of 1000 cells per well in 96-well plates, with 5 replicates of each condition. Cell viability was determined by using the CellTiter-Glo Luminescent Cell Viability Assay (Promega): cells were incubated with $100 \mu \mathrm{L}$ of the reagent for 20 minutes on a shaker, covered with a dark lid. Luciferase activity was measured by using the EnVision 2101 Multilabel Reader (PerkinElmer) on the day the cells were seeded (day 0) and daily after that for 3-4 days. The day 1 through day 4 viability measurements were normalized to that measured on day 0 . The initial seeding density of 1000 cells per well was chosen so that cells would be close to but less than $100 \%$ confluent by the final day.

\section{Crystal violet staining}

Cells were reseeded in 12-well plates at a concentration of 15,000 cells per well, with 4 replicates of each condition. After removing the media and washing with PBS, we fixed the cells in 4\% formaldehyde (Sigma-Aldrich, St. Louis, MO) and gently rocked them for 10 minutes at room temperature. The formaldehyde was then removed, and the cells were washed twice with PBS, incubated with 1\% crystal violet (Sigma-Aldrich) while gently rocking for 10 minutes at room temperature, and then rinsed with water until the water washed clear, after which $0.1 \%$ SDS was added and incubated for 10 minutes 
while gently rocking. The absorbance of each well was then measured at $590 \mathrm{~nm}$ by using a SpectraMax M5 (Molecular Devices). Crystal violet staining was performed on the same day as the initial cell seeding (day 0) and daily thereafter for 3-4 days. Crystal violet absorbance readings measured on days 1-4 were normalized to that measured on day 0 .

\section{IncuCyte proliferation assays}

Cells were reseeded in a 24-well plate at a concentration of 10,000 cells per well, with 4 replicates of each condition. Cell proliferation was monitored by using an IncuCyte live-cell imaging system (Essen BioScience, Ann Arbor, MI): 9 images were captured in each well every 12 hours. The percentage of confluent cells was calculated by using IncuCyte software.

\section{Stable cell lines}

Stable cell lines were prepared by plating 500,000 Daoy cells in 10-cm dishes. After 24 hours, cells in antibiotic-free media were transfected with $10 \mu \mathrm{g}$ of either pEZXMR03 (vector control) or pEZX-MR03-miR-124 in FuGENE6 diluted in OptiMEM. The media were replaced with normal growth media 24 hours after transfection. The cells were treated with $1 \mu \mathrm{g} / \mathrm{mL}$ puromycin 24 hours after media replacement. Puromycin was added every 3 days for 2 weeks until nontransduced control cells were completely killed by the puromycin, after which the cells were considered to be stable. Expression of miR124 in the stable cells was confirmed by using a microscope to observe the GFP signal expressed from the vector and by performing qPCR assays to quantify the levels of miR124.

\section{D-spheroid formation assay}

Parental Daoy cells and Daoy cells stably expressing miR-124 or the control vector (MR03) were seeded into a round-bottom 96-well plate at 3 different densities (288, 800, and 2500 cells/well). Media were changed every 3-4 days, and spheroid areas were calculated after 23 days by using the IN Cell Analyzer 6000 (GE). Viability was also measured on day 23 by using the CellTiter-Glo 3D Cell Viability Assay (Promega) according to the manufacturer's protocol and shown as raw luminescence units (RLU).

\section{Cerebellar granule neuron analysis}

Cerebellar granule neurons (CGNs) were prepared as described [462]. Briefly, cerebella were dissected from the brains of P7 C57BL/6 mice, and pial layers were removed; the tissue was treated with trypsin/DNase and triturated into a single-cell suspension by using fine-bore Pasteur pipettes. The suspension was layered onto a 
discontinuous Percoll gradient and separated by centrifugation. The small-cell fraction was then isolated. The resulting cultures routinely contained $95 \%$ CGNs and $5 \%$ glia. The cultures were maintained in Basal Medium Eagle (BME; Life Technologies) supplemented with glutamine and 10\% horse serum. All animal experiments were performed in accordance with a protocol approved by St. Jude Children's Research Hospital's Institutional Animal Care and Use Committee. The animals were housed at $22-23^{\circ} \mathrm{C}$ with a $12 \mathrm{~h} \mathrm{light/dark} \mathrm{cycle} \mathrm{and} \mathrm{free} \mathrm{access} \mathrm{to} \mathrm{food} \mathrm{and} \mathrm{water} \mathrm{at} \mathrm{the} \mathrm{St.} \mathrm{Jude}$ Animal Resources Center certified by the American Association for Accreditation of Laboratory Animal Care. Animals were euthanized by decapitation for the preparation of CGNs.

\section{Statistical analysis}

Results are shown as the mean \pm standard error of the mean. The sample values were compared to control values by using a two-tailed unpaired student's t-test. GraphPad Prism 6 software was used to graph results and to calculate the statistical significance.

\section{Results}

\section{Three miRNAs directly target $N R 4 A 1$}

To identify miRNAs that may target $N R 4 A 1$, we used a luciferase reporter system in which $293 \mathrm{~T}$ cells were co-transfected with a reporter plasmid containing the 3 'UTR of NR4A1 along with our collection of 296 miRNAs. In the NR4A1-3'UTR-Luc reporter, the firefly luciferase gene is directly upstream of the 3'UTR sequence: a miRNA that binds to the 3'UTR will decrease the translation of the luciferase mRNA, resulting in decreased luciferase activity being detected by the Dual-Glo Luciferase Assay System. As shown in a waterfall plot (Figure 3-1A), miR-124 caused the greatest reduction in luciferase levels among the 296 miRNAs tested. We selected 40 miRNAs that caused $40 \%$ or more reduction in luciferase activity and retested them in triplicate: 13 of the 40 miRNAs repeatedly decreased luciferase levels by $30 \%$ or more (Figure 3-1B).

In addition to using the luciferase reporter system, we used the Cancer miRNAs Transcriptome PCR Array (as described in Materials and Methods), which contains cDNA from HeLa cells transfected with one of 90 cancer-related miRNAs, many of which were included in our collection of 296 miRNAs used in the luciferase reporter screen. We found that miR-124 was one of the 3 miRNAs that substantially downregulated NR4A1 (Figure A-1).

Among the miRNAs that downregulated NR4Al (Figure 3-1A and B), only miR124, $\mathrm{miR}-15 \mathrm{a}$, and miR-224 were predicted by multiple prediction algorithms to target $N R 4 A 1$ by binding to its seed region (a 5- to 8-nucleotide sequence within the 3 'UTR that 

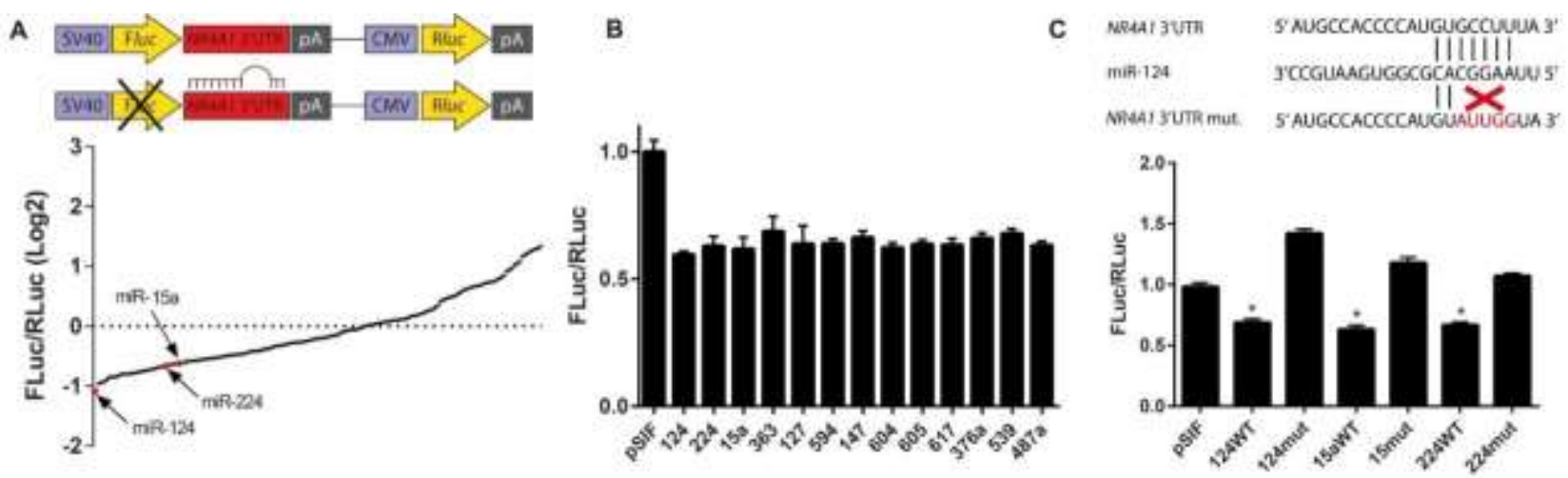

Figure 3-1. miR-124, miR-15a, and miR-224 directly target NR4A1.

(A) miR-124 caused the greatest decrease in luciferase activity after $293 \mathrm{~T}$ cells were transfected with a NR4A1-3'UTR-Luc reporter and one of 296 miRNAs. Each data point on the waterfall plot corresponds to the resulting luciferase activity for each miRNA. Renilla luciferase (RLuc) was used to normalize firefly luciferase (FLuc) activity. (B) Thirteen miRNAs significantly $(p<0.001)$ reduced luciferase activity below that of the pSIF control vector (average of 3 independent experiments is shown). (C) The seed region where miR-124, miR-15a, and miR-224 are predicted to bind within the 3'UTR of NR4A1 was mutated, and luciferase assays were performed to show direct targeting of NR4A1 by these 3 miRNAs. The data shown are the average of 3 independent experiments. $*$ indicates $p<0.001$. 
mediates the direct binding of a miRNA). These binding predictions are based on the predicted seed regions found within the TargetScan database. When we mutated the binding sites within the 3'UTR corresponding to seed regions for miR-124, miR-15a, and miR-224, the mutated 3'UTR (124mut, 15mut, and 224mut) became resistant to the corresponding miRNA (Figure 3-1C), demonstrating that these miRNAs directly target $N R 4 A 1$ by binding to a seed region within the NR4A1 3'UTR.

\section{NR4A1 is upregulated in pediatric cancer cell lines}

To further investigate the functional relationship between NR4A1 and its miRNA regulators, we first analyzed the endogenous $N R 4 A 1 \mathrm{mRNA}$ levels in several pediatric cancer cell lines. These levels were significantly higher in rhabdomyosarcoma cells lines RD, Rh41, and Rh30 than in LHCN-M2 immortalized myoblasts (Figure 3-2A). In addition, NR4A1 expression in D341 and Daoy medulloblastoma cells and in NB3 neuroblastoma cells was upregulated compared to that of $\mathrm{HCN}-2$ human cortical neurons (Figure 3-2B). We further analyzed the endogenous expression of miR-124, miR-15a, and miR-224 in Rh30, Daoy, and NB3 cells. As shown in Fig 2C, all 3 miRNAs were downregulated in Daoy, and miR-224 was decreased in NB3 cells. Interestingly, miR-124 was upregulated in NB3, a $M Y C N$-amplified neuroblastoma cell line [457], which is consistent with a recent report showing miR-124 upregulation in MYCN-amplified neuroblastoma when compared to nine other pediatric solid tumors including rhabdomyosarcoma and non- $M Y C N$-amplified neuroblastoma [463]. The mechanism responsible for the upregulation of miR-124 in NB3 cells is unclear. miR-224 was significantly downregulated in NB3 cells (Figure 3-2C), possibly contributing to the upregulation of $N R 4 A 1$ (Figure 3-2B). For the remainder of this study, we focused on the relationship between miR-124 and NR4A1 in Daoy cells because miR-124 is highly expressed in the brain [464] and it acts as a tumor suppressor in medulloblastoma [465467]. Furthermore, in undifferentiated granule neuron precursors (GNPs), the level of Nr4al was high but that of miR-124 was low (Figure A-2). Interestingly, once these GNPs differentiated, Nr4al was downregulated and miR-124 was upregulated

(Figure A-2). These observations led us to further investigate the functional relationship between miR-124 and NR4A1.

\section{miR-124 decreases NR4A1 expression}

Compared to expression in $\mathrm{HCN}-2$ cells, $N R 4 A 1$ was upregulated and miR-124 was downregulated in Daoy cells (Figure 3-3A). We further investigated the inverse correlation between NR4A1 and miR-124 expression by determining the effect of modulating miR-124 levels on the levels of NR4A1. As shown in Fig 3B, higher levels of miR-124 correlated with lower levels of NR4A1. Additionally, the NR4A1 3'UTR-Luc activity increased after treatment with a miR-124 inhibitor, further validating the inverse relationship between miR-124 and NR4A1 (Figure 3-3C). Another miR-124 inhibitor (from Exiqon) was used, yielding similar results (Figure A-3). Exogenous overexpression of miR-124 decreased the mRNA level of endogenous NR4A1 and that of 
A

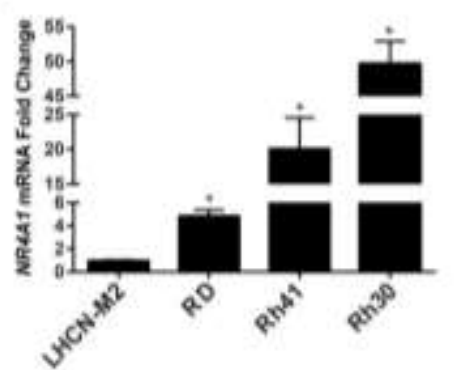

B

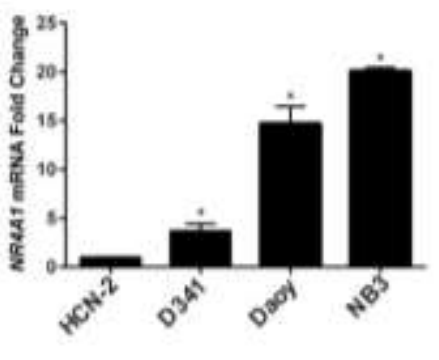

c

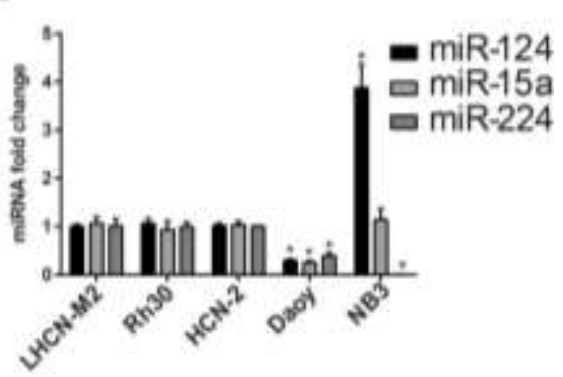

Figure 3-2. NR4A1 is upregulated in pediatric cancer cell lines.

(A, B) NR4A1 mRNA expression is upregulated in rhabdomyosarcoma, medulloblastoma, and neuroblastoma cell lines. Fold-change was calculated by normalizing the mRNA expression levels to those of their respective control cell lines (either LHCN-M2 or HCN-2), which were set to 1. (C) Endogenous miRNA expression in Rh30, Daoy, and NB3 cells shows that all 3 miRNAs are downregulated in Daoy cells and that miR-224 is downregulated in NB3 cells. RNU48 was used as an internal control. All data shown are the average of 3 independent experiments; ${ }^{*} p<0.0001$. 
A

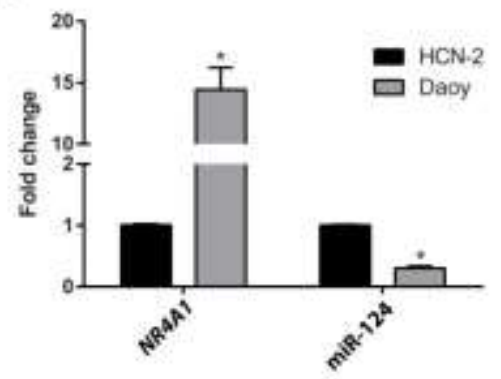

D

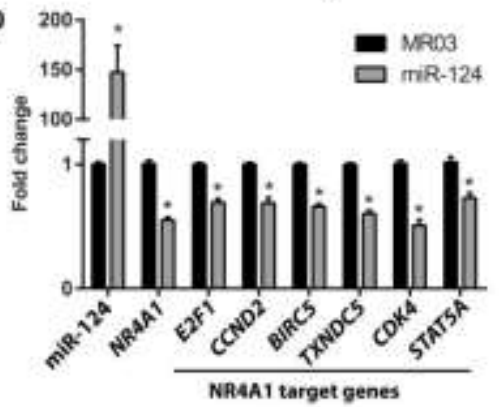

B

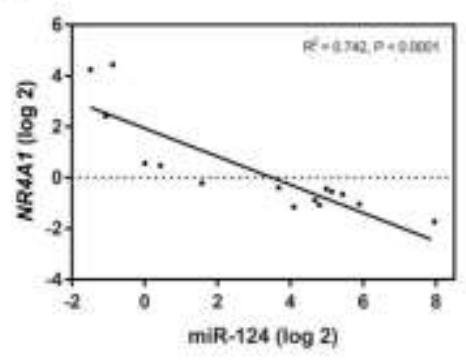

E

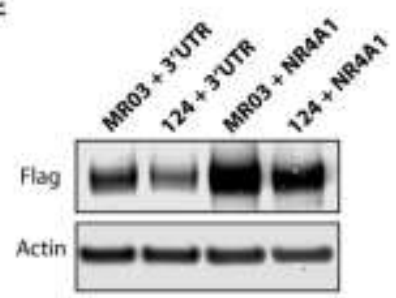

C
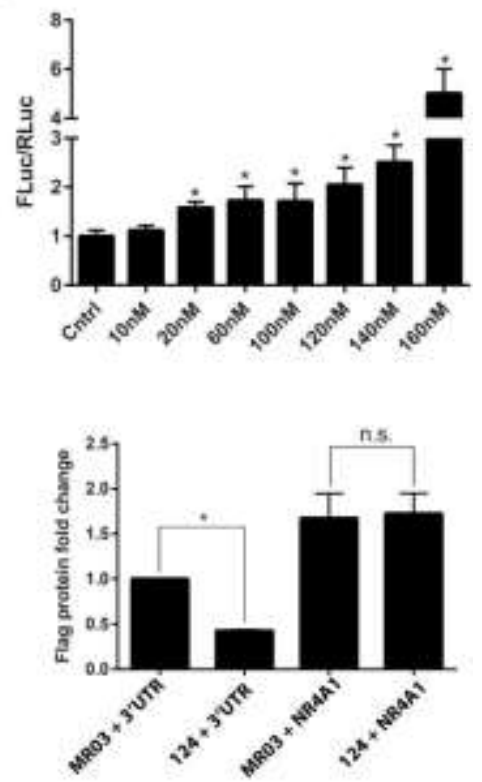

Figure 3-3. miR-124 decreases NR4A1 levels.

(A) Endogenous expression levels of miR-124 and NR4A1 were measured in Daoy cells and human cortical neurons (HCN-2). (B) NR4A1 and miR-124 expression are inversely related in Daoy cells exogenously expressing various levels of miR-124. The levels of NR4A1 and miR-124 changed in an inversely correlated manner. (C) Daoy cells were cotransfected with the NR4A1-3'UTR reporter plasmid (NR4A1-3'UTR-Luc) and either an inhibitor of miR-124 (10 - 160 nM of oligonucleotide used as indicated) or an oligo control (Cntrl) from Life Technologies; resulting luciferase levels were measured. The data shown are representative of 3 independent experiments. (D) Either miR-124 or the control vector (MR03) was exogenously expressed in Daoy cells, and the resulting levels of miR-124 and NR4A1 were measured along with the expression of NR4A1 target genes. (E) Daoy cells were co-transfected with miR-124 (124) or vector control (MR03) and Flag-NR4A1 plasmid with (3'UTR) or without (NR4A1) the 3'UTR to confirm that miR124 targets the $3^{\prime}$ UTR. Flag and actin protein levels were detected by Western blot and quantified by using ImageJ. Levels of Flag protein were first normalized to those of actin; then MR03-3UTR was set to 1, and all other samples were compared to this sample. The Western blot shown is representative of 3 independent experiments, and the bar graph shows the average protein fold-change from 3 experiments. * indicates $p<0.05$. 
several target genes of NR4A1, including E2F1, CCND2 (cyclin D2), BIRC5 (survivin), TXNDC5, CDK4, and STAT5A (Figure 3-3D). Furthermore, miR-124 overexpression also decreased the expression of NR4A1 target genes in 293T cells (Figure A-4). To demonstrate that elevated miR-124 decreases NR4A1 protein levels in a 3'UTRdependent manner we examined the effect of overexpressed miR-124 on a Flag-tagged NR4A1 construct without the $3^{\prime}$ UTR (NR4A1) or with the $3^{\prime}$ UTR (3'UTR). As shown in Figure 3-3E, overexpression of miR-124 decreased the level of Flag-NR4A1 only when the ${ }^{\prime}$ 'UTR of NR4A1 was present.

\section{NR4A1 promotes cell viability and proliferation}

Studies have shown that NR4A1 increases cell survival and proliferation in various adult cancer cell lines, suggesting an oncogenic role for NR4A1 in those particular cancers [230, 233, 239, 468-470]. We examined whether this was also the case for pediatric cancer cell lines, specifically Daoy medulloblastoma cells. Upon exogenous overexpression of NR4A1 in Daoy cells, there was increased cell viability as assessed by the CellTiter-Glo assay (Figure 3-4A). Cell proliferation was measured by performing crystal violet staining and using an IncuCyte live-cell imaging system, which monitors real-time cell proliferation. Compared to cells transduced with the empty vector (EV), the cells exogenously overexpressing NR4A1 showed increased crystal violet staining and confluence over the course of 4 days (Figure 3-4B and C). Figure 3-4D shows the elevated NR4A1 mRNA levels after transduction with NR4A1. These data support the notion that NR4A1 promotes tumor growth not only in adult cancers but also in pediatric solid tumors.

\section{Knockdown of NR4A1 decreases cell viability and proliferation}

To further validate the effects of NR4A1 on cell proliferation and viability, $N R 4 A 1$ was knocked down via pooled siRNA targeting NR4Al (siNR4A1). Daoy cells transfected with siNR4A1 exhibited decreased cell viability and proliferation as measured by the CellTiter-Glo assay, crystal violet staining, and the IncuCyte assay

(Figure 3-5A-C and E). Figure 3-5D shows the knockdown efficiency. The 4 individual siRNAs making up the pooled siRNA were then tested individually. siNR4A1_4 most efficiently knocked down $N R 4 A 1$ and caused the greatest decrease in cell viability and proliferation (Figure A-5).

\section{miR-124 decreases cell viability and proliferation}

The observations that NR4A1 promoted cell proliferation (Figures 3-4 and 3-5) and that miR-124 directly targeted and downregulated NR4A1 (Figure 3-3) led us to examine the effects of miR-124 on cell viability and proliferation. Exogenous and stable expression of miR-124 in Daoy cells substantially reduced cell viability and proliferation (Figure 3-6A-C). To measure the ability of the Daoy cells to form microtumors, we used 
A

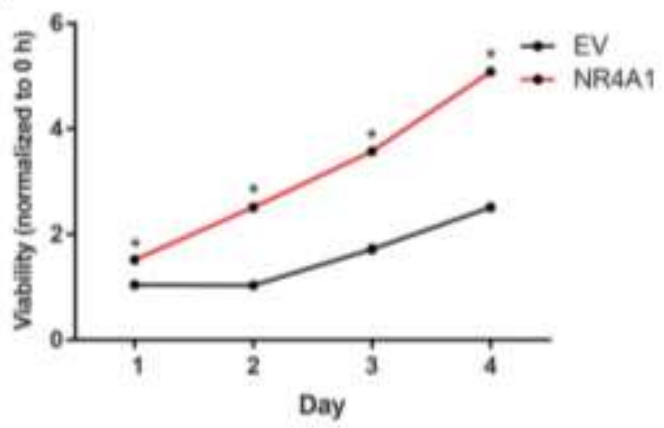

C

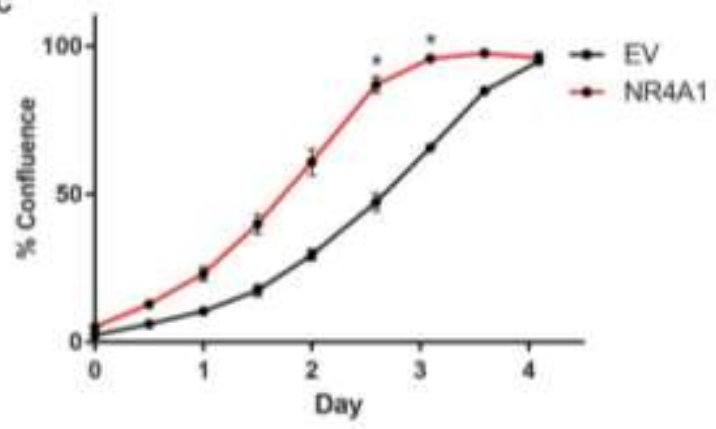

B

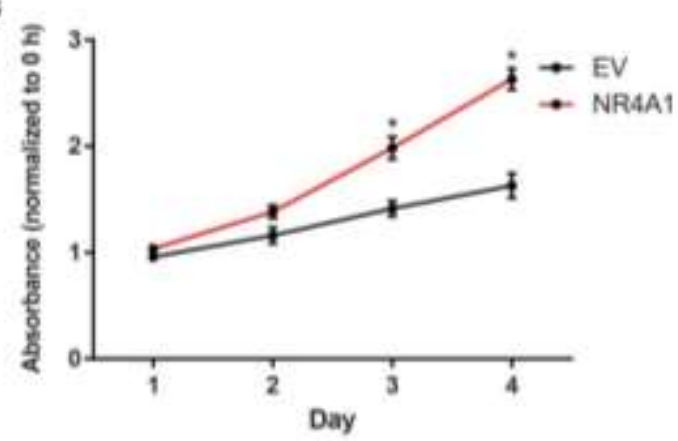

D

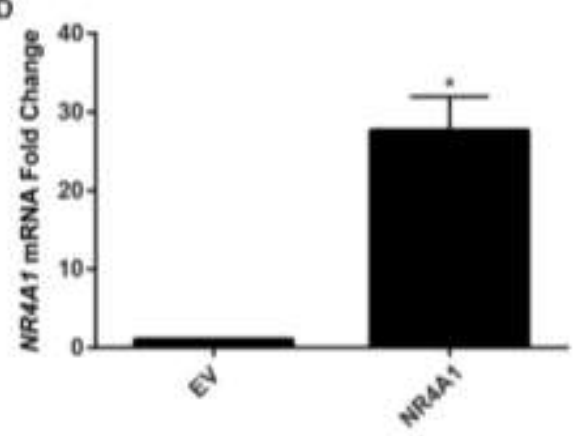

Figure 3-4. NR4A1 promotes cell viability and proliferation.

(A) Daoy cells were transduced with pSIN-NR4A1 (NR4A1) or pSIN vector (EV), and cell viability was measured via the CellTiter-Glo assay every day for 4 days. Viability for each day was normalized to that of Day 0 (0 hours), and statistical significance was calculated for each day. (B) Cells were stained with crystal violet every day for 4 days to measure proliferation over time. The absorbance was measured and normalized to that of Day 0 (0 hours). The statistical significance was calculated for each day. (C) Cell proliferation was monitored by using an IncuCyte live-cell imager for real-time imaging. The resulting cell confluence was recorded every 12 hours for 4 days. (D) NR4A1 mRNA level was measured after transduction with NR4A1. All experiments were performed by using Daoy cells transduced with EV or NR4A1 lentivirus. All data shown are representative of 3 independent experiments; $* p \leq 0.0001$. 

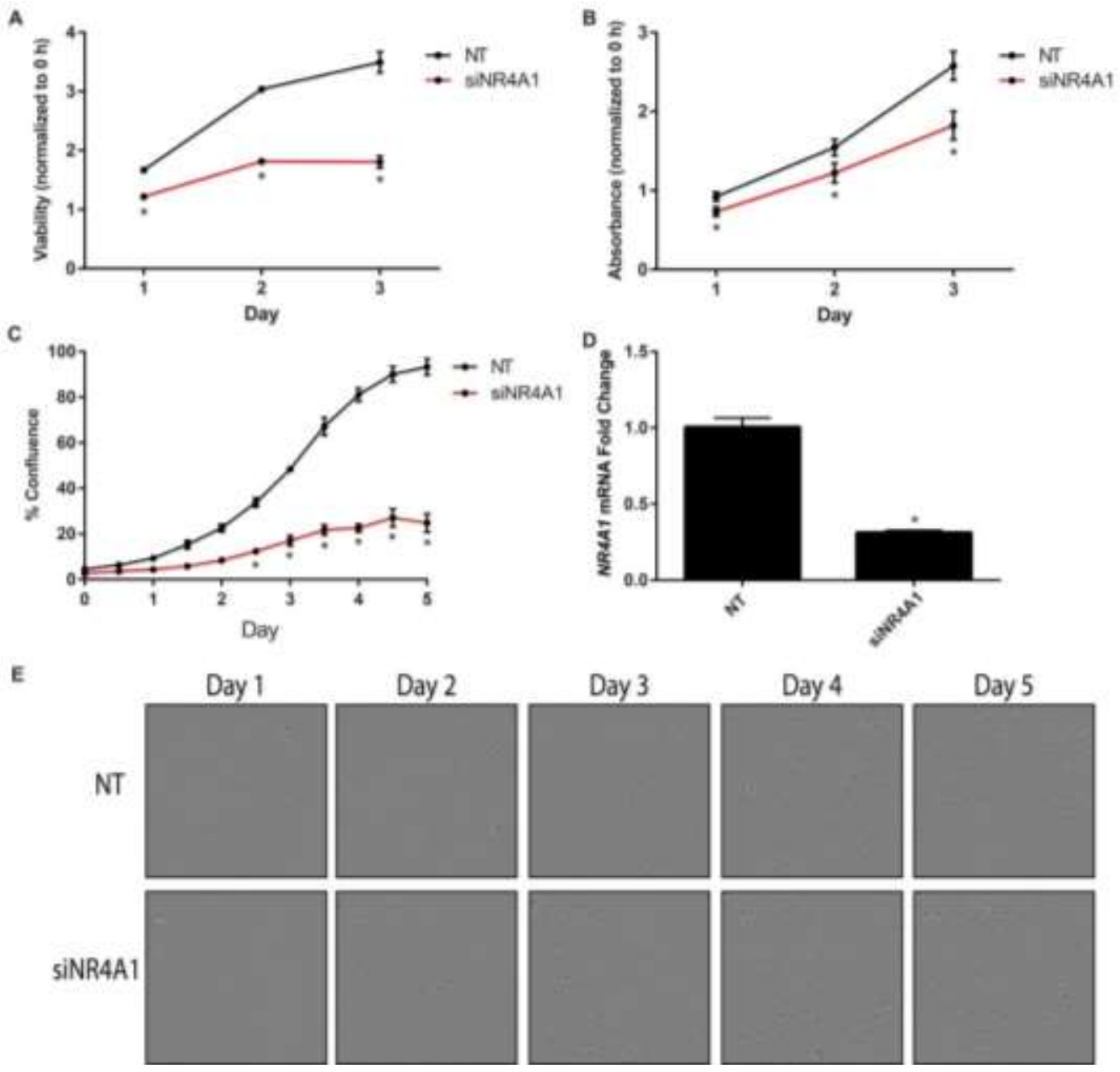

Day 5

Figure 3-5. NR4A1 knockdown decreases cell viability and proliferation.

(A) Daoy cells were transfected with $20 \mathrm{nM}$ of the SMARTpool siNR4A1 or nontargeting control (NT), and cell viability was measured via the CellTiter-Glo assay every day for 3 days. Viability for each day was normalized to that of Day 0 ( 0 hours), and statistical significance was calculated for each day; ${ }^{*} p<0.0001$. (B) Cells were stained with crystal violet every day for 3 days to measure proliferation over time. The absorbance was measured and normalized to that of Day 0 ( 0 hours). The statistical significance was calculated for each day; ${ }^{*} p<0.01$. (C) Proliferation was monitored via the IncuCyte live-cell imager. Cell confluence was averaged, with 4 replicates of each condition; * $p<0.0001$. (D) NR4A1 was significantly $(p<0.0001)$ decreased after transfecting Daoy cells with siNR4A1. (E) Images shown for each NT and siNR4A1 panel over 5 days are the same image view within the same well and are representative of 3 independent experiments with 4 wells for each condition. These images correspond to the data in C. Data shown in A are representative of 5 independent experiments; data in B are representative of 4 independent experiments, and data in $\mathrm{C}$ and $\mathrm{E}$ are representative of 2 independent experiments. Data shown in D is the average of 4 independent experiments. 

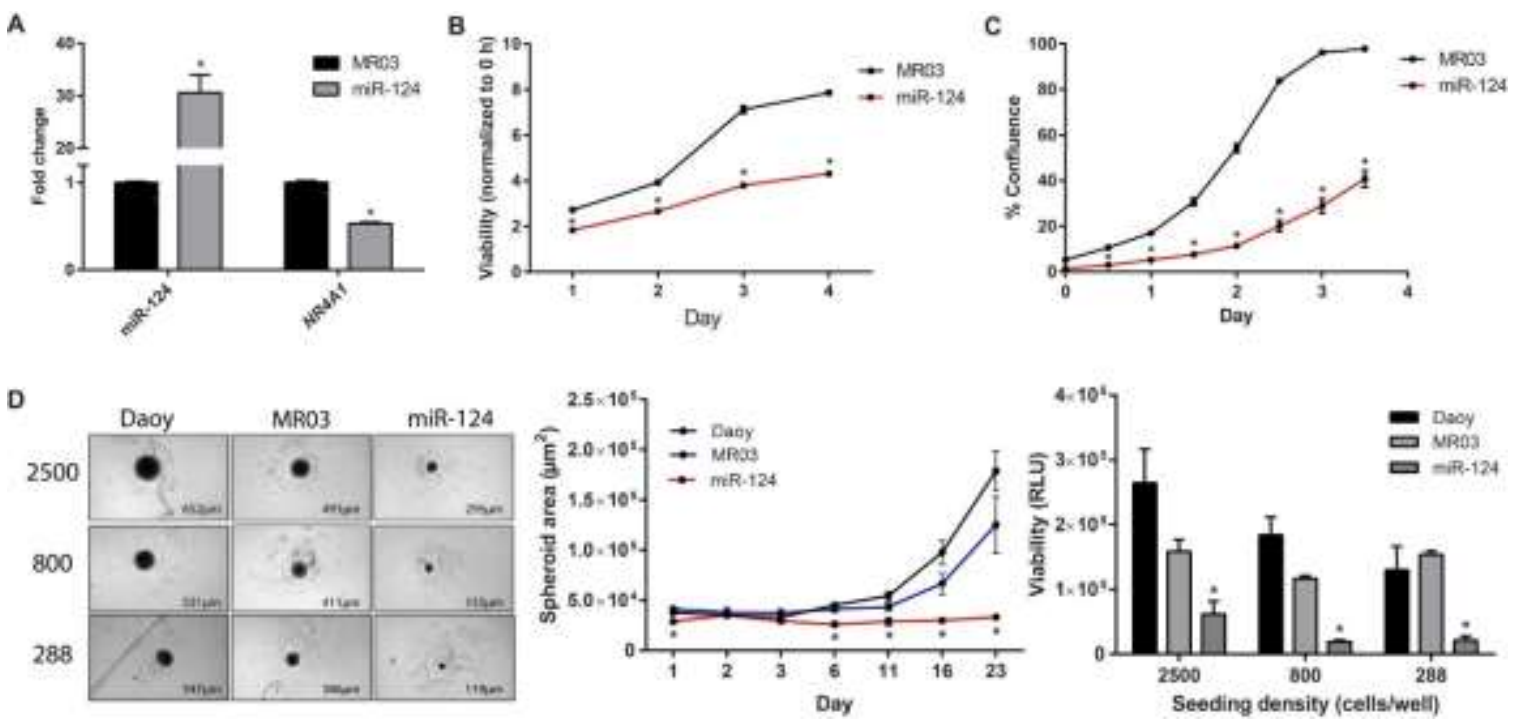

Figure 3-6. miR-124 decreases cell proliferation in 2D and 3D cultures.

(A) Expression of miR-124 was significantly $(p<0.0001)$ increased after antibiotic selection of Daoy cells transduced with pEZX-MR03-miR-124. As a result, NR4A1 mRNA levels were significantly decreased $(p<0.0001)$. Data shown are the average of 6 independent experiments. (B) The CellTiter-Glo assay was used to analyze the cell viability of Daoy cells stably expressing exogenous miR-124 or vector control (MR03). Viability for each day was normalized to that of Day 0 ( 0 hours), and statistical significance was calculated for each day; ${ }^{*} p<0.0001$. (C) Stable cells were imaged by using the IncuCyte live-cell imager to determine cell proliferation over the course of 3.5 days, and statistical significance was determined for each day; ${ }^{*} p<0.0001$. (D) Parental Daoy cells (Daoy) and Daoy cells stably expressing exogenous miR-124 (miR-124) or its control vector (MR03) were seeded at 3 densities $(288,800$, and 2500 cells/well) and grown using 3D culture techniques. After 23 days (left panel), the cells' spheroid areas $\left({ }^{*} p<0.01\right)$ were measured by using the IN Cell Analyzer (middle panel). Viability $(* p<0.05)$ was determined by performing CellTiter-Glo 3D Cell Viability Assays and is shown as raw luminescence units (RLU) (right panel). The spheroid area data shown are for cells seeded at an initial density of 800 cells per well. Data from B are representative of 5 independent experiments; data from $\mathrm{C}$ are representative of 4 independent experiments, and data from $\mathrm{D}$ are representative of 2 independent experiments. 
round-bottom 96-well plates to promote the formation of 3D spheroids. After allowing the cells to form spheroids for 23 days, we found that cells exogenously overexpressing miR-124 underwent less spheroid growth and viability than did the control cells (Figure 3-6D). These results are consistent with previously reported evidence showing the negative effects of miR-124 on medulloblastoma cell growth [465-467].

\section{Conclusions}

To our knowledge, our study is the first to show that the level of NR4A1 is regulated by miR-124 and that NR4A1 has roles in proliferation of pediatric cancer cells such as Daoy medulloblastoma cells. Most published research on NR4A1 as a therapeutic target involves using drugs to induce NR4A1-mediated apoptosis; our discovery that miR-124 regulates NR4A1 suggests that it may be possible to modulate NR4A1 and influence cancer cell growth by regulating miR-124.

In a panel of pediatric cancer cell lines, we found $N R 4 A 1$ to be upregulated in all cell types including RD, Rh41, and Rh30 RMS cells, D341 and Daoy medulloblastoma cells, and NB3 neuroblastoma cells. We identified 3 miRNAs that directly target NR4A1, including miR-124, miR-15a, and miR-224, all of which were downregulated in Daoy, demonstrating an inverse correlation between NR4A1 and the miRNAs that target it. However, we did not observe this trend in the other cell types. There are most likely additional miRNAs that target NR4Al in the other cell types and perhaps this is why we did not see a correlation.

It is difficult to conclusively determine the role of NR4A1 in medulloblastoma based on these studies as Daoy cells are not classified as any of the 4 subtypes of medulloblastoma. Furthermore, there is not substantial evidence in expression databases demonstrating whether or not NR4A1 is upregulated in patients with medulloblastoma. Therefore, further studies showing expression levels of NR4A1 in medulloblastoma are required in order to make this determination. Furthermore, tumorigenicity assays such as soft agar colony formation assays that test anchorage independent growth can be performed in multiple medulloblastoma cell types that more closely resemble the different subtypes of medulloblastoma. The tumorigenic potential of NR4A1 can also be tested in vivo wherein NR4A1 can be overexpressed in a medulloblastoma cell line that is then injected into mice and the resulting tumors are measured to determine if NR4A1 promotes the growth of medulloblastoma tumors. Additionally, knockdown studies can be conducted in vivo to conclusively confirm whether or not NR4A1 affects the growth rate of medulloblastoma tumors.

Interestingly, we found that $N R 4 A 1$ expression decreases while miR-124 expression increases in differentiating GNPs. This supports previous research showing that miR-124 expression is higher in differentiated GNPs compared to undifferentiated GNPs. The decrease in NR4A1 expression suggests that NR4A1 must be downregulated, possibly by miR-124, in order for differentiation to occur. It is of interest to explore the function of NR4A1 in GNPs as these are the main type of neuron in the cerebellum where 
a subtype of medulloblastoma originates. A more thorough discussion on this topic can be found in Chapter 5.

In short, we found that miR-124 targets and decreases NR4A1 expression and function. NR4A1 promotes cell proliferation in Daoy medulloblastoma cells, but miR124 reduces it, in part by targeting $N R 4 A 1$ and decreasing its transcriptional activity. This results in decreased expression of NR4A1 target genes important in cell cycle progression and survival. This study supports the use of miRNA mimics to treat cancers, especially those in which NR4A1 has an oncogenic role. 


\section{CHAPTER 4. THE ROLE OF NR4A1 IN SKELETAL MUSCLE DIFFERENTIATION}

\section{Introduction}

Diseases related to muscle such as Duchenne muscular dystrophy and rhabdomyosarcoma, which are prevalent in children, are in desperate need of effective treatments. In order to develop therapies, it is critical to understand normal muscle development and function. The process of muscle formation, termed myogenesis, involves several stages including proliferation, migration, and differentiation. During the first stage, mesodermal progenitors exit the cell cycle and several transcription factors appear including Pax3, Pax7, Myf5, and MyoD1. These transcription factors are required for the cells to become myoblasts. These myoblasts will then align with one another and become myocytes after the expression of additional transcription factors including myogenin (MYOG) and Myf6. During the final stages of differentiation, the myocytes will fuse to form multinucleated myotubes, which will then become muscle fibers. Additional muscle markers such as myosin heavy chain (MHC/MYH) and muscle creatine kinase (CKM) become expressed in the later stages of differentiation [471-473].

NR4A1 has many physiological roles in various tissues such as its involvement in muscle function. For example, Nr4al promotes glucose and oxidative metabolism in skeletal muscle and has even been shown to promote myofiber size and muscle mass in mice [148, 474, 475]. However, the role of NR4A1 during human skeletal muscle differentiation is not well understood. For these studies, we utilized primary SkMC and HSMM as well as immortalized LHCN human skeletal muscle cells. In this study we found that $N R 4 A 1$ expression increases during the myogenic program in LHCN, and knockdown of NR4A1 results in decreased expression of myogenic markers in all 3 cell types. This suggests that NR4A1 participates in skeletal muscle differentiation.

In addition, ZNF148 may regulate $N R 4 A 1$ during myogenesis by suppressing its expression. Knockdown of ZNF148 in LHCN rapidly induced the formation of myotubes with a resulting increase in $N R 4 A 1$ expression. Understanding these mechanisms may be beneficial in cancer since certain malignancies such as rhabdomyosarcoma result from the failure of cells to differentiate and instead proliferate uncontrollably, leading to tumor formation and cancer progression.

\section{Methods}

\section{Cell culture}

LHCN-M2 (LHCN) cells are human skeletal myoblasts [458] and were grown in flasks coated with extracellular matrix (0.2\% MaxGel ECM in PBS from Sigma-Aldrich) and cultured in DMEM supplemented with 15\% FBS, $0.02 \mathrm{M}$ HEPES, $0.03 \mu \mathrm{g} / \mathrm{mL}$ zinc 
sulfate, $1.4 \mu \mathrm{g} / \mathrm{mL}$ vitamin B12, $0.055 \mu \mathrm{g} / \mathrm{mL}$ dexamethasone, $2.5 \mathrm{ng} / \mathrm{mL}$ hepatocyte growth factor (recombinant human), $10 \mathrm{ng} / \mathrm{mL}$ basic fibroblast growth factor, and 1\% penicillin-streptomycin (Life Technologies, Carlsbad, CA). Differentiation was induced by the addition of differentiation media, which consisted of DMEM supplemented with

$0.02 \mathrm{M}$ HEPES, $0.03 \mu \mathrm{g} / \mathrm{mL}$ zinc sulfate, $1.4 \mu \mathrm{g} / \mathrm{mL}$ vitamin B12, $10 \mu \mathrm{g} / \mathrm{mL}$ insulin, 100 $\mu \mathrm{g} / \mathrm{mL}$ apo-transferrin, and $1 \%$ penicillin-streptomycin. Human skeletal muscle cells (SkMC) and myoblasts (HSMM) were purchased from Lonza (Walkersville, MD) and originate from gestational tissue and adult tissue, respectively. They were cultured in SkGM-2 Skeletal Muscle Cell Growth Medium-2 (BulletKit from Lonza) and were differentiated by adding 2\% horse serum to DMEM/F-12 media (Lonza). All cells were cultured at $37^{\circ} \mathrm{C}$ in $5 \% \mathrm{CO} 2$.

\section{Transfection}

LHCN were plated at 100k cells/well in 6-well plates coated with ECM. After 24 hours they were transfected with $10 \mathrm{nM}$ non-targeting control (NT) or siRNA for NR4A1 (siNR4A1) and $5 \mathrm{ul} \mathrm{RNAiMAX} \mathrm{(in} 300 \mathrm{ul}$ of OptiMEM). Media was replaced with fresh media 24 hours after transfection. Differentiation media was added once the cells reached $100 \%$ confluence, which was typically 72 hours post-transfection. Media was replaced every 2 days during the course of differentiation. For knockdown of ZNF148, cells were transfected with $10 \mathrm{~nm}$ control (Cntrl) or siZNF148 (Cat. \# D-012658-04). Reagents for siRNA were purchased from Dharmacon using their siGENOME siRNA (GE Healthcare, Lafayette, CO). For both siNR4A1 and siZNF148, the knockdown efficiency was approximately $90 \%$, and 3 out of 4 of the individual siRNAs had a similar phenotype, indicating that they are specific to their respective target genes. SkMC and HSMM were plated at 150k cells/well in 6-well plates and were transfected 48 hours after plating. Once cells reached $70-80 \%$ confluence, differentiation was induced by the addition of differentiation media, which was replaced every 2 days.

\section{RNA extraction and $q P C R$}

RNA was extracted by using the Maxwell 16 LEV simplyRNA Tissue Kit in conjunction with the Maxwell 16 Research Instrument (Promega). The resulting RNA concentration was measured by using the NanoDrop and was subsequently converted to cDNA by using the SuperScript VILO cDNA Synthesis Kit (Life Technologies). Target gene mRNA expression was detected by using specific Taqman probes (20X) and quantitated via the 7900HT Fast Real-Time PCR System (Applied Biosystems). 18S (Assay ID Hs99999901_s1) was used as an endogenous control for all gene expression analysis, including NR4A1 (Assay ID Hs00374226_m1), MYOG (Assay ID Hs01072232_m1), MYH2 (Assay ID Hs00430042_m1), CKM (Assay ID Hs00176490_m1), and ZNF148 (Assay ID Hs01070570_m1). 


\section{Protein isolation and Western blot analysis}

Cell pellets were lysed in Pierce RIPA lysis buffer (Thermo Fisher Scientific, Grand Island, NY) and incubated on ice for 30 minutes prior to sonication for $5 \mathrm{~s}$ and finally centrifugation at $17,500 \mathrm{~g}$ for 5 minutes. The protein concentration was measured by using a Pierce BCA Protein Assay kit and absorbance was measured at $540 \mathrm{nM}$ by using the SpectraMax M5 microplate reader (Molecular Devices, Sunnyvale, CA). The protein lysate was mixed with 10X loading buffer and 4X LDS (Life Technologies) and incubated at $95^{\circ} \mathrm{C}$ for 5 minutes. Subsequently, the protein mixture was loaded into a NuPAGE $4-12 \%$ Bis-Tris gel (Life Technologies) and run at $100 \mathrm{~V}$ for 2 hours. The protein was then transferred to a PDVF membrane using the slow transfer method. This method involves activation of the PDVF membrane with methanol for 10 minutes followed by incubation in transfer buffer to remove the methanol. The protein was transferred to the PDVF membrane overnight on ice at 0.45 amperes. The blot was then blocked at room temperature for one hour by using Odyssey blocking buffer (LI-COR Biosciences, Lincoln, NE). Mouse monoclonal anti-MHC (produced using hybridoma cells from the Developmental Studies Hybridoma Bank; used at 1:200 dilution), mouse monoclonal anti-myogenin (Santa Cruz; used at 1:500), and mouse monoclonal anti- $\beta$ actin (Sigma; A5441; used at 1:2000 dilution) antibodies were added and incubated overnight at $4^{\circ} \mathrm{C}$. After the primary antibodies were removed, the blot was washed with TBST three times for 15 minutes each time prior to incubation with the secondary antibody (LI-COR Biosciences; used at 1:10,000 dilution) for 1 hour at room temperature. Subsequently, the blot was washed three times with TBST and proteins were detected by using the Odyssey imaging system (LI-COR Biosciences).

\section{Immunofluorescence}

Cells were washed 3 times with PBS and fixed with 2\% paraformaldehyde for 15 minutes at room temperature followed by additional washes with PBS. The cells were then permeabilized with $0.1 \%$ Triton X-100 for 15 minutes followed by 3 washes with PBS. The monolayer was then washed 5 times with $0.5 \%$ BSA diluted with PBS (PBB) and blocked with $2 \%$ BSA for 45 minutes followed by 5 washes with PBB. The primary antibody for myosin heavy chain 1 (MHC) was added at a $1: 100$ dilution overnight at $4{ }^{\circ} \mathrm{C}$ and then washed 5 times with PBB. The secondary antibody was used at a 1:500 dilution and incubated for 1 hour at room temperature followed by 5 washes with PBB. DAPI was added at a 30,000 dilution of $1 \mathrm{mg} / \mathrm{ml}$ for 5 minutes followed by 5 washes with cold PBS. The chambers were then removed and mounted with coverslips and mounting media and imaged using a Nikon Eclipse Ti microscope.

\section{Statistical analysis}

Results are shown as the mean \pm standard error of the mean. Significance was determined by using a two-tailed unpaired student's t-test to compare the sample values 
to the control values. GraphPad Prism 6 software was used to graph results and to calculate the statistical significance.

\section{Results}

\section{NR4A1 expression increases during LHCN myogenesis}

To determine if NR4A1 plays a role during myogenesis, expression was analyzed during the differentiation of LHCN, SkMC, and HSMM human skeletal muscle cells. NR4A1 mRNA levels increased by about 4 fold during the differentiation of LHCN, however it did not substantially increase in SkMC and HSMM cells. To verify that the cells were indeed differentiated, the myogenic markers MYOG, MYH2, and CKM were measured (Figures 4-1, 4-2, and 4-3). MYOG is considered an early marker whereas MYH2 and CKM are late differentiation markers. In order to determine if the other NR4A family members are playing a role, the mRNA levels of $N R 4 A 2$ and $N R 4 A 3$ were measured in all 3 cell types. NR4A2 was greatly decreased in LHCN and SkMC but not in HSMM, whereas $N R 4 A 3$ was increased in all 3 cell types. This suggests that NR4A2 may inhibit differentiation while NR4A3 may promote it.

\section{NR4A1 knockdown delays differentiation}

To determine the significance of the increased NR4AI expression in LHCN, NR4Alwas knocked down via siRNA (siNR4A1). As a result, differentiation was severely delayed as shown by the decrease in differentiation markers and the visible lack of myotube formation (Figure 4-4B-D). Protein levels for MYOG and MHC were also extremely decreased in the cells lacking NR4AI (Figure 4-4E). Bright-field images of the cells clearly show well-differentiated NT cells by Day 4 compared to the siNR4A1 cells that did not form myotubes (Figure 4-4F). Interestingly, $N R 4 A 1$ expression was induced up to 15 fold by Day 5 in the cells transfected with the non-targeting (NT) control (Figure 4-4A). This is a much larger induction compared to the untransfected LHCN in Figure 4-1A. Similar results were observed in SkMC and HSMM, however the NR4A1 mRNA levels actually decreased during differentiation in the NT cells

(Figures 4-5 and 4-6).

\section{ZNF148 knockdown induces differentiation}

Studies from our lab have demonstrated that knockdown of the transcription factor ZNF 148 rapidly induces differentiation in LHCN cells, suggesting that ZNF148 plays an inhibitory role during myogenesis. One study has shown that ZNF148 can suppress the expression of $N R 4 A 1$ by directly binding to its promoter [446]. To determine the functional significance of this inhibitory mechanism, ZNF148 was knocked down via siRNA in LHCN cells which resulted in the rapid formation of myotubes by Day 2 
A

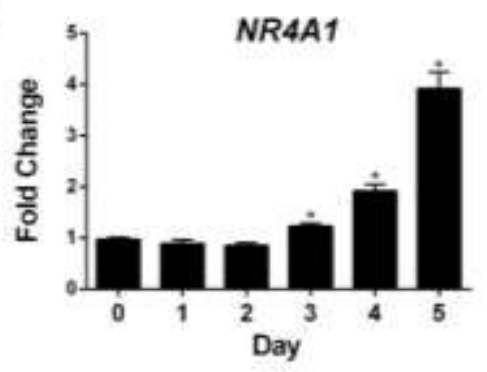

B

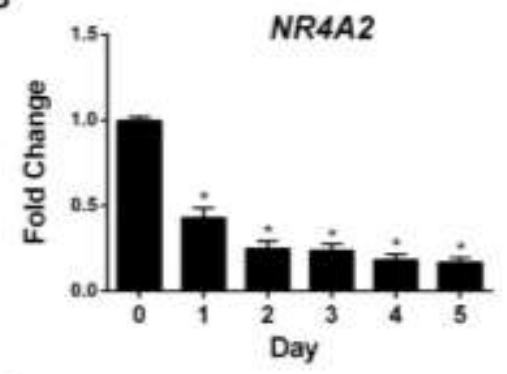

C

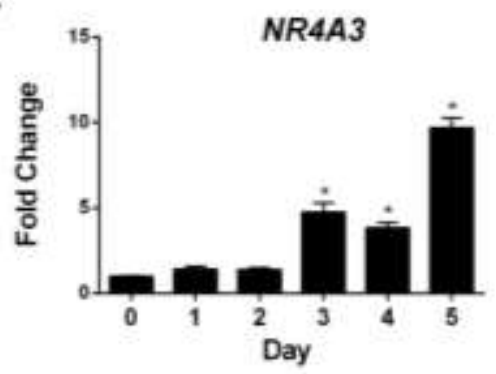

D

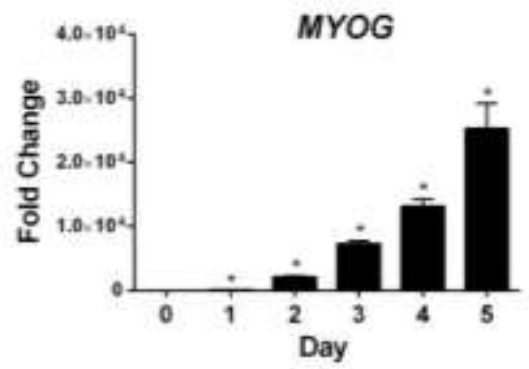

E

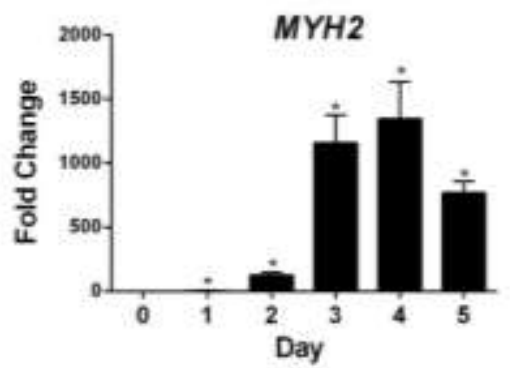

$\mathbf{F}$

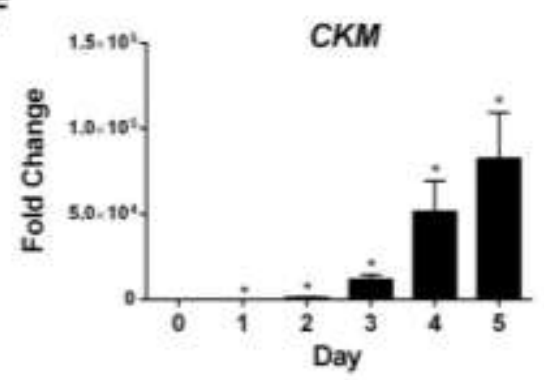

Figure 4-1. NR4A1 expression increases during LHCN differentiation.

(A-F) LHCN were differentiated for 5 days with cells collected for each day beginning on Day 0 when differentiation media is added. The mRNA levels for $N R 4 A 1, N R 4 A 2$, $N R 4 A 3$, myogenin $(M Y O G)$, myosin heavy chain (MYH2), and muscle creatine kinase $(C K M)$ were measured and normalized to Day 0, which was set to 1. Data shown is the average of at least 2 independent experiments. * indicates $p<0.01$. 
A

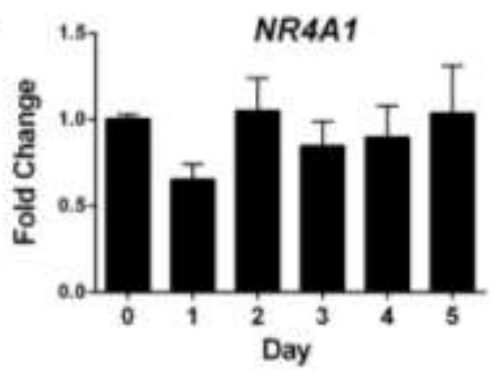

B

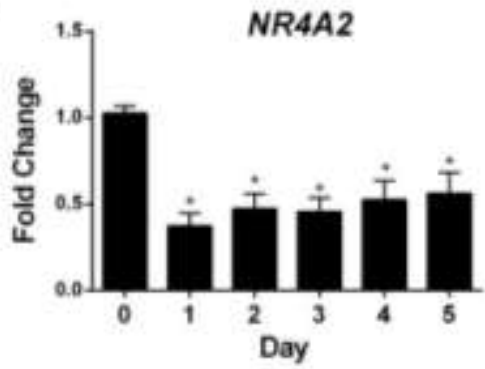

c

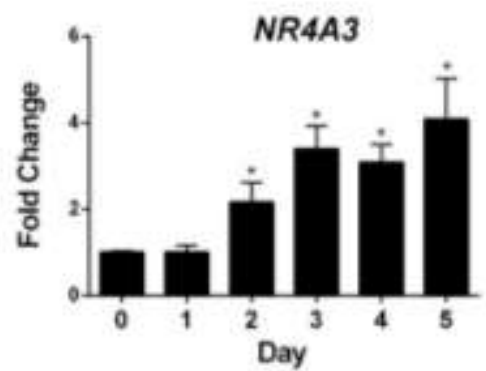

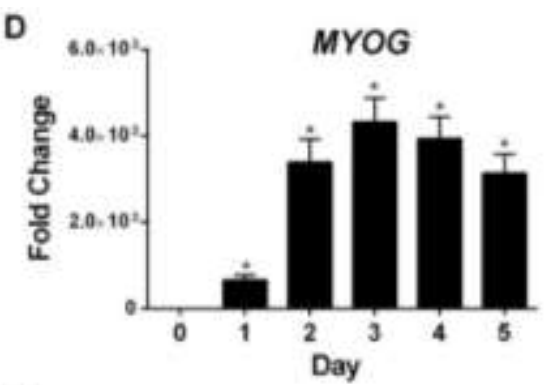

E

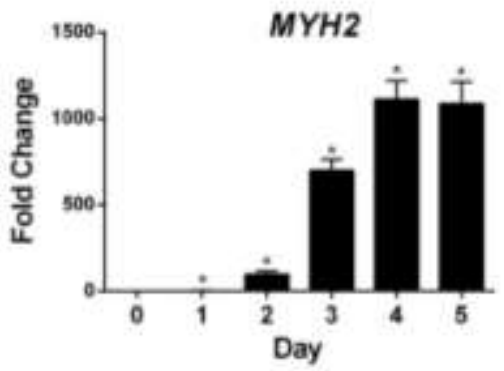

$\mathbf{F}$

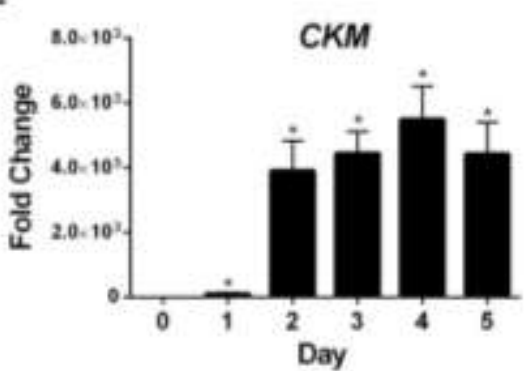

Figure 4-2. NR4A1 expression does not increase during SkMC differentiation. (A-F) SkMC were differentiated for 5 days with cells collected every day beginning on Day 0 when differentiation media was first added. The mRNA levels of NR4A1, NR4A2, NR4A3, MYOG, $M Y H 2$, and $C K M$ were measured and normalized to Day 0, which was set to 1 . Data shown is the average of 5 independent experiments. * indicates $p<0.05$. 
A

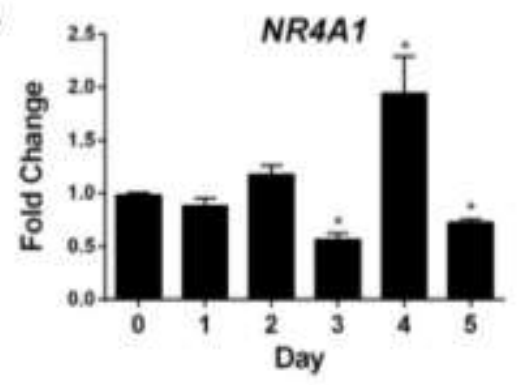

B

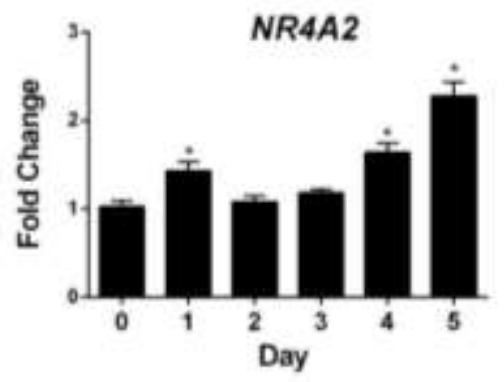

C

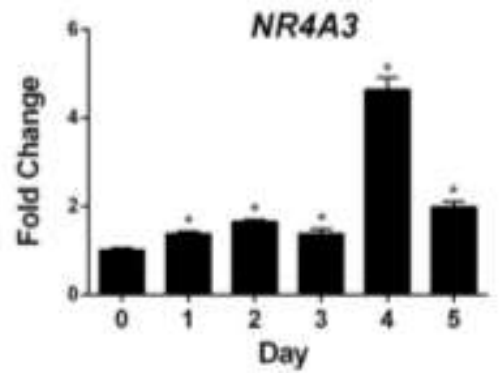

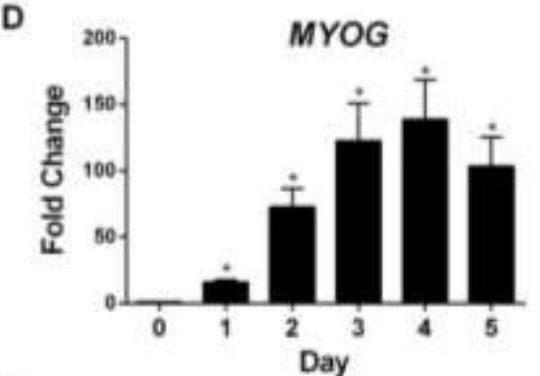

$\mathbf{E}$

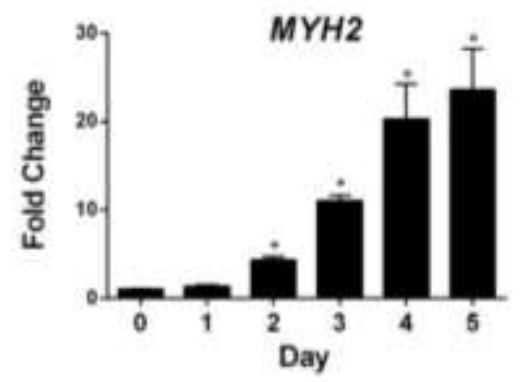

$\mathbf{F}$

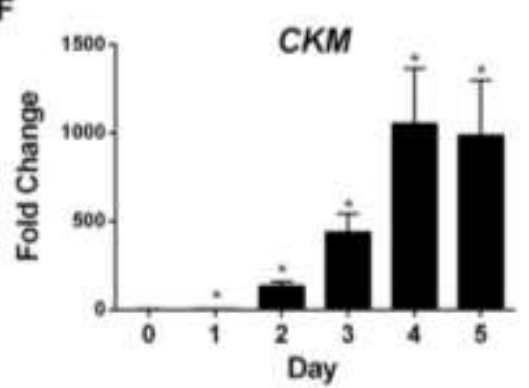

Figure 4-3. NR4A1 expression does not increase during HSMM differentiation. (A-F) HSMM were differentiated for 5 days with cells collected every day beginning on Day 0 when differentiation media was first added. The mRNA levels of $N R 4 A 1, N R 4 A 2$, NR4A3, MYOG, MYH2, and CKM were measured and normalized to Day 0, which was set to 1 . Data shown is the average of at least 2 independent experiments. * indicates $p<0.05$. 
A

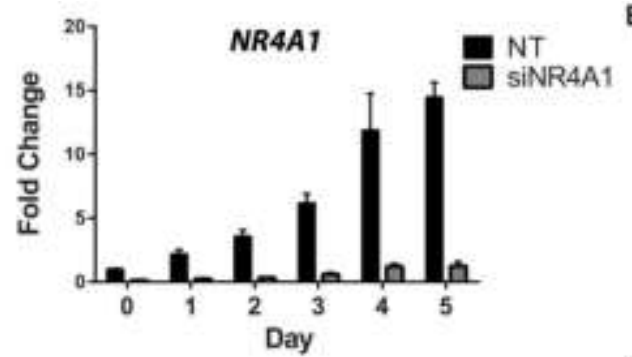

C

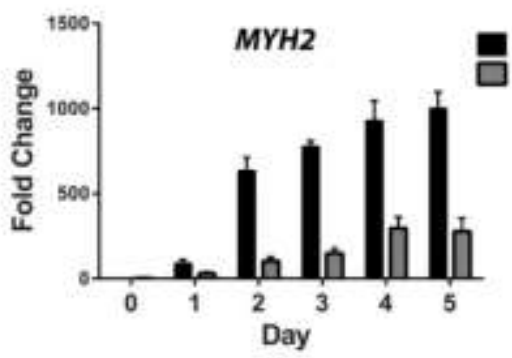

E

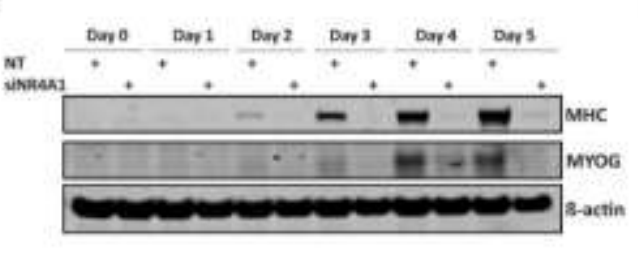

B

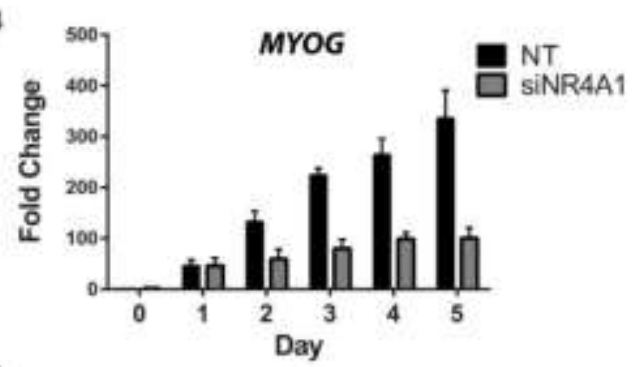

D

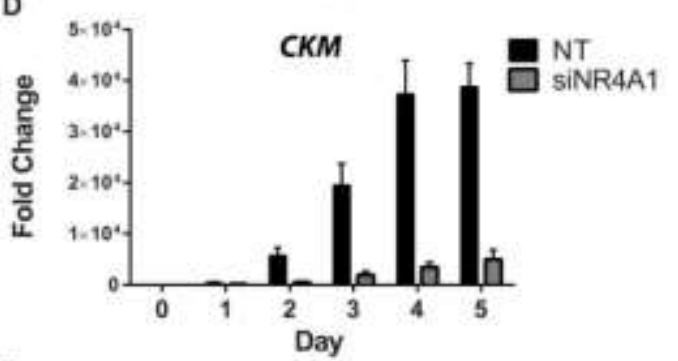

$\mathrm{F}$

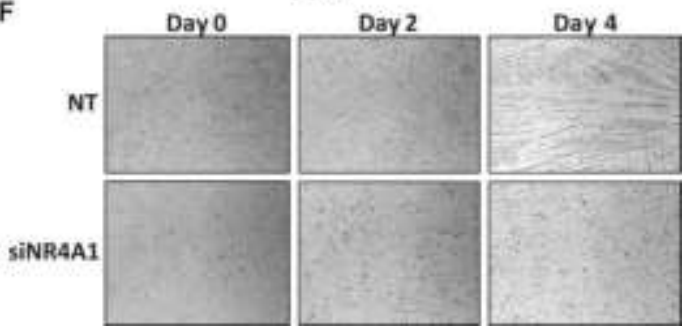

Figure 4-4. Knockdown of NR4A1 delays differentiation in LHCN.

(A-D) LHCN were transfected with $10 \mathrm{~nm}$ non-targeting control (NT) and siRNA for NR4A1 (siNR4A1) prior to differentiation for 5 days with cells collected every day beginning on Day 0 . The mRNA levels of NR4A1 and differentiation markers were measure and normalized to NT Day 0, which was set to 1 . Data shown is the average of 3 independent experiments. (E) Protein levels for MHC, MYOG, and $\beta$-actin were detected in a western blot. (F) Bright-field images with 10X magnification were taken on Days 0, 2 , and 4 with representative images shown. 
A

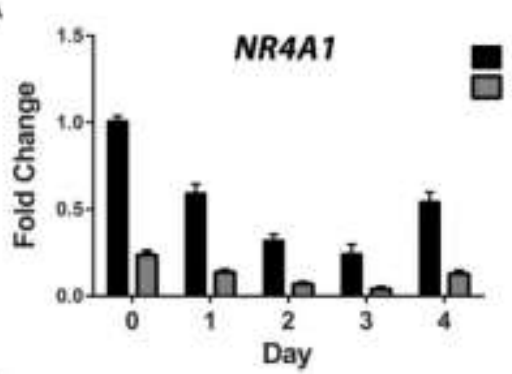

C

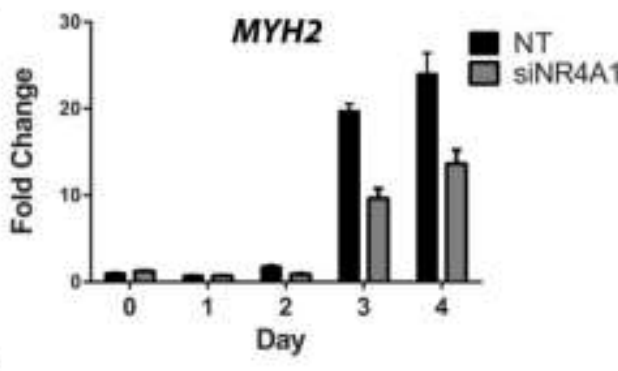

$\mathrm{E}$

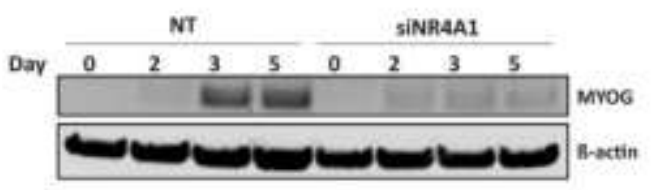

B
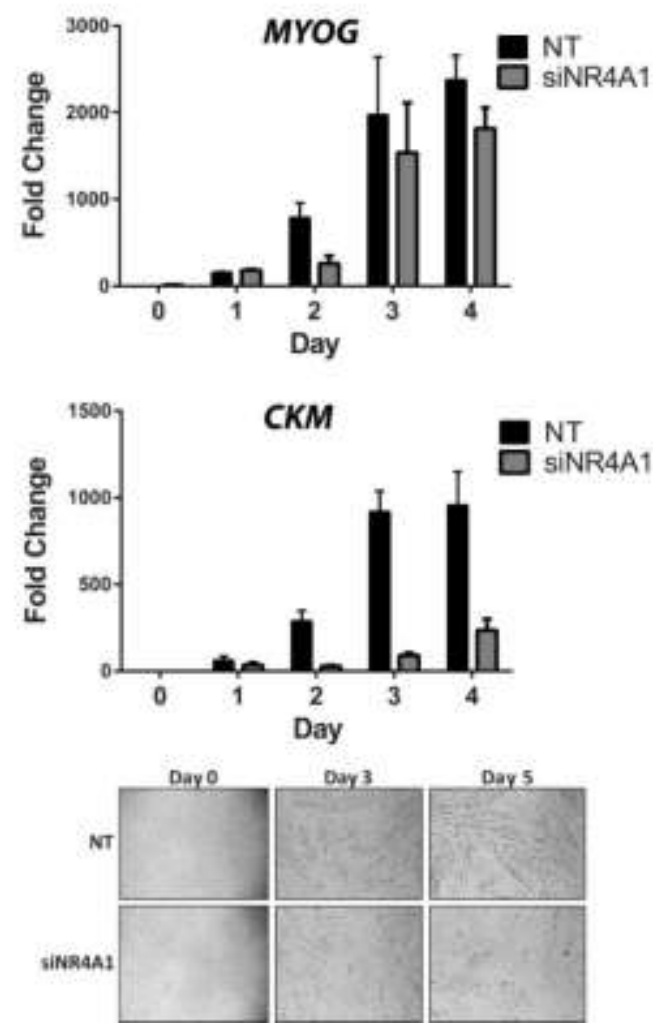

Figure 4-5. NR4A1 knockdown delays differentiation in SkMC.

(A-D) SkMC were transfected with $10 \mathrm{~nm}$ NT and siNR4A1 prior to differentiation for 5 days. The mRNA levels of NR4A1 and differentiation markers were measured and normalized to NT Day 0, which was set to 1. Data shown is the average of 3 independent experiments. (E) Protein levels of MYOG and $\beta$-actin were measured in a western blot. (F) Bright-field images at 10X magnification were taken on Days 0, 3, and 5 with representative images shown. 
A

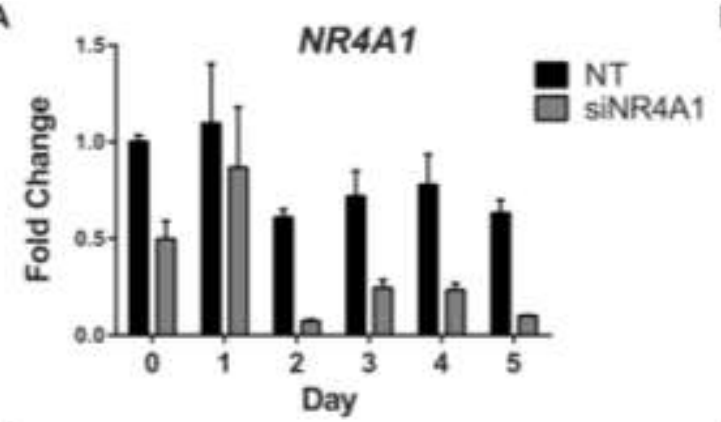

C

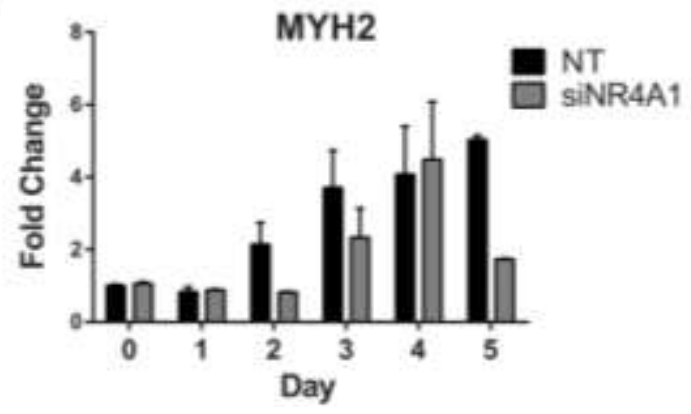

B

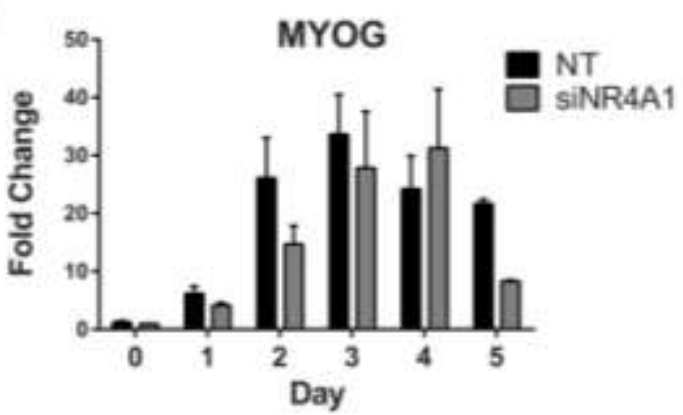

D

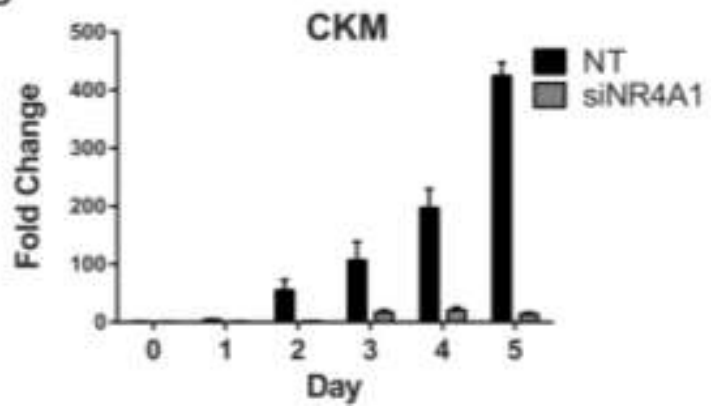

Figure 4-6. NR4A1 knockdown delays differentiation in HSMM.

(A-D) HSMM were transfected with $10 \mathrm{~nm}$ NT and siNR4A1 prior to differentiation for 5 days. The mRNA levels of NR4A1 and differentiation markers were measured and normalized to NT Day 0, which was set to 1. Data shown is the average of 2 independent experiments. 
(Figure 4-7C-D). Interestingly, knockdown of ZNF148 induced differentiation in the presence of growth media, demonstrating the strong repressive power of endogenous ZNF148 in the control cells (Figure 4-7A). Accordingly, NR4Al expression was also induced more than 15 fold in the cells with siZNF148 under growth conditions and more than 10 fold under differentiation conditions (Figure 4-7B).

\section{Conclusions}

In all, we discovered a novel role for NR4A1 in skeletal muscle differentiation. $N R 4 A 1$ expression increases during the myogenic program in LHCN, and knockdown of NR4A1 results in decreased expression of differentiation markers in LHCN, SkMC, and HSMM human skeletal muscle cells. This supports a previous study showing that $\mathrm{Nr} 4 \mathrm{al}$ increases during the differentiation of mouse C2C12 skeletal muscle cells [142, 476]. However in that study, they noted that differentiation still occurred in the absence of Nr4al. Perhaps the difference in our results depends on the species and cell type as those studies were conducted in murine cells whereas these studies were performed in human cells.

Although NR4Al was increased in LHCN, it was not increased in SkMC or HSMM. This could be an artifact of the immortalized state of LHCN, whereas SkMC and HSMM are primary cells. We would expect NR4A1 to have similar expression patterns in LHCN and HSMM since these two cell types both originate from adult tissue, whereas SkMCs are isolated from gestational tissue. Another difference in cell type is that SkMCs are precursors and therefore less differentiated, whereas HSMM and LHCN are myoblasts committed to becoming muscle. Even though $N R 4 A 1$ was not increased in SkMC and HSMM, knockdown of NR4A1 in these cells still resulted in decreased expression of myogenic markers. Therefore it would appear that NR4A1 is important during myogenesis. It was also interesting to observe the higher induction of NR4A1 in cells transfected with NT. Perhaps there are some off target effects of the NT that inadvertently increase $N R 4 A 1$ levels. It would be reasonable to test additional NT controls to determine if these effects are specific to the original NT used here. Furthermore, we found that $N R 4 A 2$ was decreased during the differentiation of LHCN and SkMC while $N R 4 A 3$ was increased in all 3 cell types. This suggests that $N R 4 A 2$ overexpression should hypothetically inhibit differentiation while $N R 4 A 3$ overexpression should promote it. Perhaps the NR4A family has synergistic effects where myogenesis is enhanced upon NR4A2 knockdown and NR4A1 and NR4A2 overexpression.

Since there are several stages of differentiation, it is of interest to determine the stage in which NR4A1 is acting. Perhaps it is promoting the initial stages of cell cycle arrest, or maybe it is mainly functioning during a later stage. One way to elucidate this would be to determine the differentiation and fusion indices. The differentiation index is a measure of the number of nuclei in myosin-positive cells which indicates how differentiated the cells are whereas the fusion index is a measure of the number of nuclei in each myotube, indicating the ability of myoblasts to fuse into myotubes [477]. Calculating these indices would help determine the effect of NR4A1 on how well the 
A

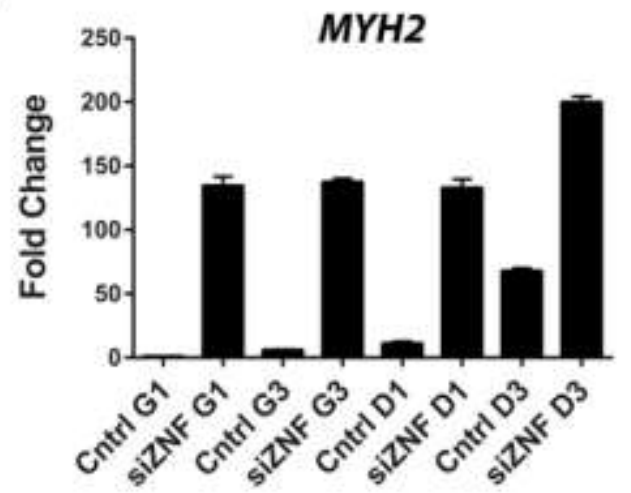

C

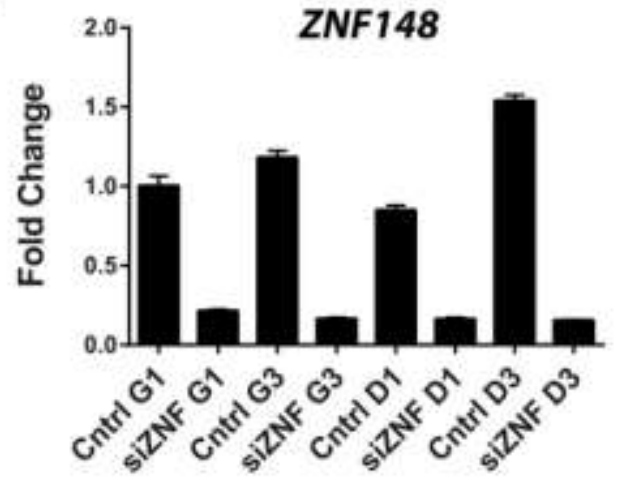

B

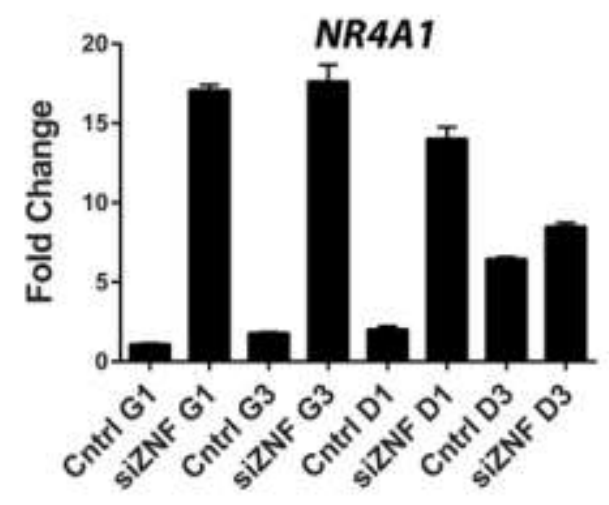

D
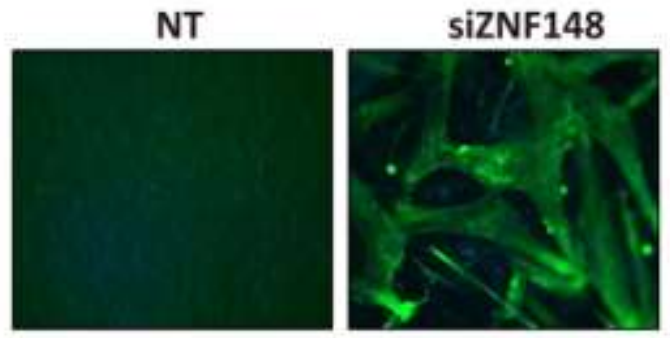

Figure 4-7. Knockdown of ZNF148 rapidly induces differentiation in LHCN. (A-C) LHCN cells were transfected with $10 \mathrm{nM}$ Cntrl or siZNF and cultured in either growth $(\mathrm{G} 1, \mathrm{G} 3)$ or differentiation (D1, D3) media with cells collected on Days 1 and Day 3. The resulting mRNA levels of $M Y H 2, Z N F 148$, and NR4A1 were measured and normalized to Cntrl G1, which was set to 1 . (D) Immunofluorescence was performed to detect MHC (green) and DAPI (blue) in cells with ZNF148 knocked down. Images shown are $20 \mathrm{X}$ magnification and were taken 2 days after the addition of differentiation media. 
cells are able to differentiate and fuse. It is possible that knocking down NR4A1 is blocking or delaying myoblast fusion without effecting differentiation.

On the other hand, NR4A1 may actually be more involved in the metabolism aspect. Upon knockdown of NR4A1 in LHCN, SkMC, and HSMM, the muscle marker $C K M$ is dramatically downregulated whereas the other muscle markers $M Y O G$ and MYH2 are only modestly decreased. Many studies have shown that CKM is an essential enzyme during energy homeostasis and mediates the synthesis of creatine phosphate (CrP) and ADP by transferring the gamma phosphate from ATP. During intense exercise when there is a high demand for energy, CKM will then synthesis ATP from CrP and ADP [478]. As expected, decreased CKM contributes to the gradual loss of muscle mass and function during aging in vivo $[478,479]$. As mentioned, NR4A1 has been shown to promote glucose and oxidative metabolism in murine skeletal muscle [148, 474].

Therefore, it is quite possible that the role of NR4A1 in muscle is primarily related to metabolism. To confirm this, expression of additional genes involved in metabolism should be analyzed upon knockdown or overexpression of NR4A1 during the differentiation of LHCN, SkMC, and HSMM cells.

Interestingly, knockdown of ZNF148 resulted in elevated levels of $N R 4 A 1$ as well as rapid formation of myotubes, even in the absence of differentiation growth factors. This most likely occurs by the direct repression of $N R 4 A 1$ by ZNF148 at the promoter of $N R 4 A 1$ as previously shown in pancreatic $\beta$-cells. This demonstrates a possible mechanism by which ZNF148 and NR4A1 may be controlling skeletal muscle differentiation. These results also highlight ZNF148 as a therapeutic target in diseases such as RMS where the cells have failed to differentiate. 


\section{CHAPTER 5. DISCUSSION*}

\section{Regulation of $N R 4 A 1$ by miRNAs in Cancer}

The regulation of $N R 4 A 1$ by miRNAs was previously unknown, and the function of NR4A1 in pediatric cancers is currently undetermined. In this study, we found that miR-124 directly targets NR4A1 and that NR4A1 is upregulated in multiple pediatric cancer cell lines, including rhabdomyosarcoma, neuroblastoma, and medulloblastoma cell lines. We focused on elucidating the function of NR4A1 and miR-124 in medulloblastoma cells, and showed that exogenous expression of miR-124 in Daoy medulloblastoma cells decreased the cells' proliferation and viability.

Several previous reports suggest that miR-124 might also regulate NR4A1 indirectly. In pancreatic beta-cells, Sp1 binds to the promoter of NR4A1 and increases NR4A1 levels [480]. Interestingly, during neuronal differentiation of mesenchymal stem cells, miR-124 targets Sp1 mRNA and decreases Sp1 expression [481]. The results of these studies suggest that miR-124 may indirectly decrease NR4A1 expression by decreasing Sp1. In addition, NR4A1 binds to the promoter of several target genes, including E2F1, CCND2 (cyclin D2), BIRC5 (survivin), TXNDC5, CDK4, and STAT5A [231, 233, 438, 439]. Consistent with these reports, we showed that overexpression of miR-124 decreased expression of these 6 target genes. By promoting expression of these genes, NR4A1 exerts its effects on cell proliferation and survival. Aberrant overexpression of NR4A1 can, therefore, lead to tumor growth and cancer progression. Figure 5-1 summarizes our discovery of the miR-124/NR4A1 functional relationship in the context of relevant previous reports.

Medulloblastoma is a highly malignant primary brain tumor that originates in the cerebellum. It is also the most common malignant brain tumor in children, with patients having a 50\%-80\% chance of survival depending on the specific tumor type and other factors [482, 483]. There are 4 subgroups of medulloblastoma: Wnt, Shh, Group 3, and Group 4 [484]. A study profiling miRNAs in Shh MB tumors found 30 miRNAs that were downregulated in tumors with high Gli1, one of which was miR-124 [485]. Another miRNA profiling study also found that miR-124 in a Shh MB mouse model was downregulated compared to that in 1-month-old cerebella [486]. Furthermore, a study profiling 19 human medulloblastomas found that miR-124 was downregulated in the Wnt- and Shh-associated MBs [487]. Additionally, one study of miRNA profiles in 34 human primary medulloblastomas found that miR-124 was downregulated (subtypes were not specified) [488].

*Modified with permission from PLOS ONE. Tenga, A., et al., Regulation of Nuclear Receptor Nur77 by miR-124. PLOS ONE, 2016. 11(2): p. e0148433. 


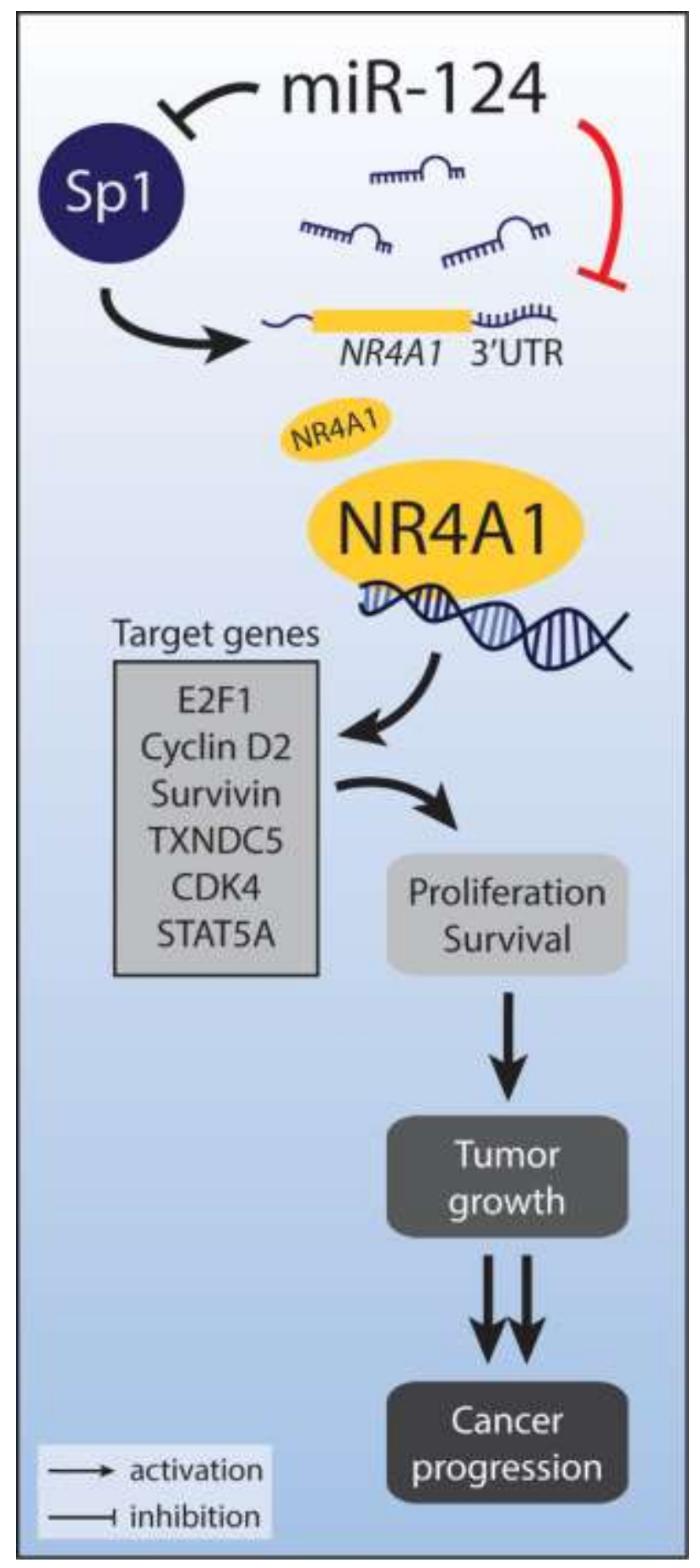

Figure 5-1. Overview of NR4A1 regulation by miR-124.

NR4A1 can be directly targeted by miR-124, as revealed by our studies reported here (indicated by the red line), or indirectly affected by miR-124 via Sp1. NR4A1 may act through several downstream target genes to promote cell proliferation and survival. 
Interestingly, miR-124 is the most abundant miRNA in the brain [464] and functions to promote neuronal differentiation [489], regulate neural stem cells [490], and induce differentiation in glioma stem cells [491]. Pierson et al. first showed that miR-124 targets CDK6 in medulloblastoma cell lines and that miR-124 is downregulated in medulloblastoma cells lines and tumors [465]. Silber et al. went on to show that miR-124 inhibits proliferation of medulloblastoma cells via cell-cycle arrest during G1 and that these results are more dramatic in cells with higher CDK6 levels. Importantly, inducible overexpression of miR-124 in vivo significantly reduced tumor growth generated by subcutaneous injection of D425 medulloblastoma cells [467]. Li et al. also found downregulation of miR-124 in 29 medulloblastomas and showed that miR-124 targets SLC16A1, which functions to efflux lactic acid during aerobic glycolysis. The authors suggest that inhibition of SLC16A1 by miR-124 decreases intracellular $\mathrm{pH}$ to a lethal level, leading to the observed growth inhibition in medulloblastoma cell lines upon overexpression of miR-124 [466]. It is clear that miR-124 has an important tumorsuppressive role in medulloblastoma and that it acts through various target genes. Our research provides, for the first time, an additional target gene of miR-124, NR4A1, which has known oncogenic roles in adult solid tumors.

As summarized in Figure 5-1, miR-124 may also indirectly downregulate NR4A1 by directly targeting the mRNA of Sp1, resulting in reduced Sp1 and NR4A1 levels. Additionally, miR-124 is predicted to target $C C N D 2$ and $T X N D C 5$, which are both target genes of NR4A1. Furthermore, miR-124 is predicted to target $R X R A(\mathrm{RXR} \alpha)$ and $G S K 3 B$ (GSK3 $\beta$ ). RXR $\alpha$ and NR4A1 heterodimerize and either translocate to the mitochondria to induce apoptosis or bind to the promoters of NR4A1 target genes to modulate transcription $[162,175]$. GSK3 $\beta$ suppresses NR4A1 activity by phosphorylating NR4A1 in colorectal cancer [418]. Therefore, it is possible for miR-124 to exert both positive and negative effects directly and indirectly on NR4A1, depending on the specific cellular context.

The main type of neuron that makes up the cerebellum is the granule neuron. We found an inverse expression pattern whereby $N R 4 A 1$ is upregulated and miR-124 is downregulated in Daoy medulloblastoma cells and in undifferentiated murine GNPs. Similar to Daoy medulloblastoma cells, in undifferentiated GNPs, the level of NR4A1 is high and that of miR-124 is low. Once the GNPs differentiate into mature granule neurons, NR4A1 levels drop dramatically and miR-124 expression increases. These observations are consistent with those in studies showing that miR-124 promotes neuronal differentiation [489-491] and that miR-124 levels in 1-month-old mouse cerebellar tissue are higher than those in P6 GNPs [486]. The dramatic decrease in $N R 4 A 1$ expression after differentiation suggests that the levels of $N R 4 A 1$ need to be reduced before the cells can develop into mature neurons.

It is reasonable to hypothesize that increased levels of miR-124 are needed to decrease NR4A1 for differentiation to occur. Aberrant downregulation of miR-124 might block differentiation and promote tumorigenesis, warranting the future investigation of the regulation of miR-124 levels. The miR-124 promoter has been reported to be hypermethylated in pancreatic cancer [492], hepatocellular carcinoma [493, 494], 
ulcerative colitis, [495] and acute lymphoblastic leukemia [496]. It is therefore of interest to analyze the promoter of miR-124 before and after differentiation to identify any changes in methylation status and to identify proteins that may bind to the promoter of miR-124, thereby affecting endogenous levels of miR-124.

NR4A1 can reportedly enhance neuronal outgrowth and differentiation: both dibutyryl-cAMP (dbcAMP) and trichostatin A (TSA) promote neurite outgrowth in PC12 rat pheochromocytoma cells by inducing $\mathrm{Nr} 4 a \mathrm{l}$ expression via acetylated Lys 14 of histone H3, and knockdown of Nr4al inhibits dbcAMP and TSA-induced neurite outgrowth [497, 498]. NR4A1 overexpression also promotes neurite formation in PC12 cells [498]. However, the opposite phenotype is observed in a murine macrophage cell line. Oxidized low-density lipoprotein (oxLDL) induces mature macrophages to differentiate into dendritic cells and induces NR4A1 expression in vascular cells. However, when NR4A1 is overexpressed in RAW264.7 murine macrophages, differentiation into dendritic cells is inhibited in the presence of oxLDL [499]. This comes back to the key point that NR4A1 functions are heavily dependent on cellular context, so it is possible for NR4A1 to have opposing functions in different tissues and cell types.

Daoy cells are classified as desmoplastic cerebellar medulloblastoma [500]; however, researchers have found that this cell line does not mimic any of the 4 subtypes of medulloblastoma. It would be useful to compare the expression level of NR4A1 in the brain to that in medulloblastoma tumors, but unfortunately there are not substantial published data showing NR4A1 levels in human medulloblastoma. However, two databases show low basal expression of NR4A1 in healthy cerebellum. The Brain Transcriptome Database shows that in situ hybridization images of the cerebellum have very little NR4A1 signal [501]. In addition, the Genotype-Tissue Expression project found that expression levels of NR4A1 in different parts of the brain, including the cerebellum, were much lower than those in other normal tissue types [502]. Given our finding of elevated NR4A1 in Daoy cells, NR4A1 may have an oncogenic role in medulloblastoma, which is supported by our data showing that exogenous overexpression of NR4A1 promotes Daoy cell viability and proliferation and that NR4A1 knockdown results in the opposite phenotype.

Upon overexpression of miR-124 in Daoy cells, NR4A1 mRNA and protein levels and the mRNA levels of NR4A1 target genes decreased, showing that miR-124 affects not only NR4A1 expression but also the transcriptional activity of NR4A1. Stable overexpression of miR-124 led to decreases in cell viability, cell proliferation, and microtumor spheroid size, suggesting therapeutic potential for miR-124 in treating cancer.

\section{Role of NR4A1 in Skeletal Muscle Differentiation}

NR4A1 appears to play a variety of roles in muscle, including the enhancement of glucose and oxidative metabolism in $\mathrm{C} 2 \mathrm{C} 12$ mouse myoblasts and in vivo in mouse and 
rat muscle [148, 474]. In addition, older studies have shown that $\mathrm{Nr} 4 \mathrm{al}$ expression increases during $\mathrm{C} 2 \mathrm{C} 12$ differentiation [142]. These cells still retained the potential to differentiate when $\mathrm{Nr} 4 \mathrm{al}$ was knocked down, however several genes involved in lipolysis were decreased. This led to the conclusion that NR4A1 is important for energy expenditure and has potential therapeutic value in treating obesity [142]. More recently, NR4A1 was found to promote myofiber size and muscle mass in vivo through activation of the Akt-mTOR-S6K cascade [475]. After global and muscle-specific knockout of Nr4al, the muscle mass was decreased. Importantly, primary myoblasts from these mice formed fewer and smaller myotubes [475]. The above studies were all performed using either animal models or murine $\mathrm{C} 2 \mathrm{C} 12$ cells. We wanted to determine the function of NR4A1 during skeletal muscle differentiation using human cell lines.

During the differentiation of LHCN, we found that $N R 4 A 1$ expression increases by about 4 fold. These results are consistent with previous studies showing a 3-5 fold increase of $\mathrm{Nr} 4 \mathrm{al}$ in $\mathrm{C} 2 \mathrm{C} 12$ cells $[142,476]$. It is of interest to determine which stage NR4A1 is becoming expressed. Since NR4A1 expression does not increase until Day 3 of differentiation, it would appear that its presence is not crucial for the initiation of differentiation. However when NR4A1 was knocked down via siRNA, the induction of differentiation was severely delayed alongside decreased expression of muscle markers in LHCN, SkMC, and HSMM. It is also possible that the fusion of myoblasts is being blocked or delayed. Thus we will need to determine exactly which stage NR4A1 is playing a role. Nonetheless, this suggests that NR4A1 is important during the differentiation of skeletal muscle cells.

Previous studies in our lab have demonstrated that the zinc finger transcription factor ZNF148 (ZBP-89) acts as a suppressor of the myogenic program as deduced from knockdown studies showing rapid formation of myotubes in LHCN cells when ZNF148 was expressed at extremely low levels. As a well-known repressor of transcription, ZNF148 is likely acting by binding to the promoters of genes involved in myogenesis and inhibiting their expression. A recent study links ZNF148 to NR4A1 by demonstrating the ability of ZNF148 to bind the promoter of $N R 4 A 1$ and block its expression in pancreatic $\beta$-cells [480]. This discovery led us to question whether ZNF148 is transcriptionally regulating NR4A1 during skeletal muscle differentiation. Upon knockdown of ZNF148 via siRNA in LHCN cells, $N R 4 A 1$ expression was rapidly induced by 15 fold along with high levels of $M H C$. This suggests that ZNF148 represses $N R 4 A 1$ in skeletal muscle cells and that downregulation of ZNF148 by siRNA relieves this repression. To confirm this, we will need to perform ChIP and probe for ZNF148 at the promoter of NR4A1 in LHCN cells.

Furthermore, it is unclear whether NR4A1 induction is due to the knockdown of $Z N F 148$ or as a result of induced differentiation. To elucidate this, we knocked down both $Z N F 148$ and NR4A1 and tested whether siZNF148 was still able to induce differentiation. If ZNF148 functions through NR4A1, we would expect abnormal differentiation in the absence of NR4A1. However, we found that differentiation was still induced at the same rate (data not shown). Therefore, it would appear that the mechanism of siZNF148-mediated differentiation is not solely through NR4A1. Since ZNF148 is a 
transcription factor and is known to target and suppress numerous genes, it may still be acting through NR4A1 in addition to many other genes. Therefore, knockdown of NR4A1 alone would not be sufficient to rescue this phenotype. Another member of the NR4A family, NR4A3, may be compensating for the lack of NR4A1. Our study among other studies has shown that NR4A3 increases during skeletal muscle differentiation and therefore it may have redundant roles with NR4A1 [503]. This would not be out of the ordinary as other studies have shown overlapping roles for NR4A1 and NR4A3 in other tissues. It is of interest to determine if $N R 4 A 3$ expression increases upon $N R 4 A 1$ knockdown. Perhaps NR4A3 partially rescues the effects of NR4A1 knockdown and therefore differentiation still occurs, albeit at a slower rate. In addition, a double knockdown of both $N R 4 A 1$ and $N R 4 A 3$ may have a more profound effect on differentiation by completely preventing the initiation of differentiation. A recent study found that knockdown of $N R 4 A 3$ in HSMM blocked the formation of myotubes, suggesting that NR4A3 is required for skeletal muscle differentiation [504].

The regulation and function of NR4A1 during differentiation is of interest. Although NR4A1 does not appear to be a mechanism for ZNF148, that does not mean that ZNF148 is not a mechanism for NR4A1. ZNF148 may need to be suppressed during differentiation in order for $N R 4 A 1$ expression to increase. We found that ZNF148 mRNA levels are unchanged during differentiation (data not shown), however its activity may still be suppressed. A proteomics study performed in our lab shows that ZNF148 protein levels decrease by almost $25 \%$ during $\mathrm{LHCN}$ differentiation (data not shown). Reduced ZNF148 activity would result in the inability to transcriptionally inhibit $N R 4 A 1$, which would result in increased $N R 4 A 1$ expression. The mechanism by which NR4A1 potentially enhances differentiation is likely through transcriptional activation of genes important for the initiation and progression of the myogenic program as depicted in Figure 5-2. NR4A1 has been shown to induce expression of CKM, although it apparently does not bind the promoter [476]. Furthermore, 685 genes were recently identified by ChIP-seq as being directly regulated by NR4A1 in AML cells [505]. Interestingly, NR4A1 was found to transcriptionally activate ENO3 [505], a gene that our lab identified in the proteomics study to increase by 26 fold during muscle differentiation. ENO3 has been previously shown to increase during differentiation and has roles during muscle development [506]. Furthermore, ENO3 was found to be regulated by ETS transcription factors, which were recently discovered to interact with NR4A1 to transcriptionally activate NR4A1 target genes [505]. Therefore, it is quite possible that NR4A1 enhances myogenesis by promoting the expression of genes involved in this process.

It may appear difficult to find a connection between the two main projects discussed here. The proliferative role of NR4A1 in cancer seems contradictory to its promyogenic role in skeletal muscle. The key to these differential functions may depend on the expression levels of NR4A1. For instance in cancer, NR4Al appears to be highly elevated. This substantial upregulation may be necessary for the proliferative effects of NR4A1. Conversely, NR4A1 expression increases during skeletal muscle differentiation (only in LHCN), although not to the same degree as in cancer. During normal muscle differentiation, NR4A1 may be acting as a co-driver to ensure the smooth and timely 


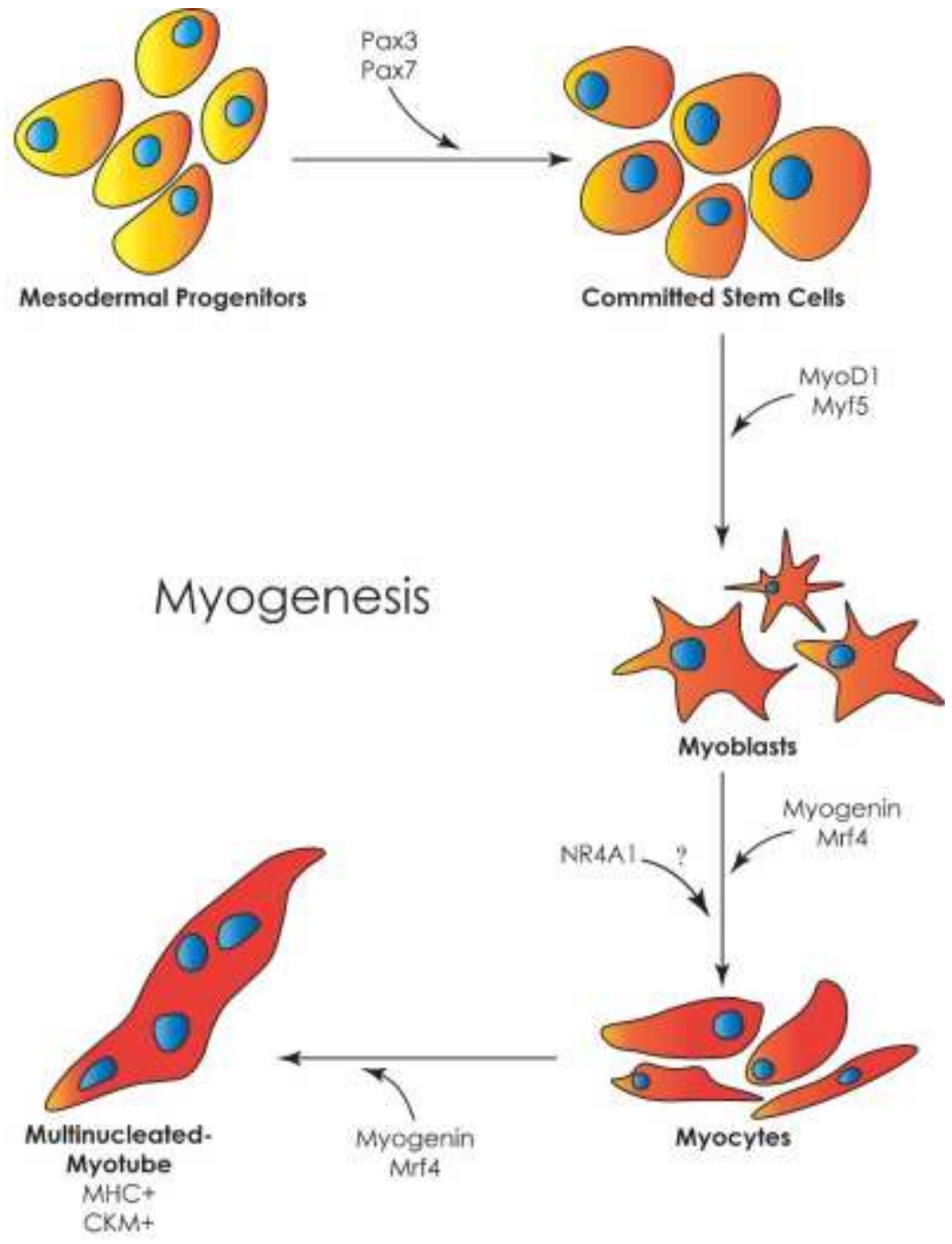

Figure 5-2. Overview of the myogenic program.

Muscle differentiation begins with mesodermal progenitors that express Pax3 and Pax7. Transcriptional activity by Myf5 allows the cells to either progress into brown adipose or into myoblasts after the addition of MyoD activity. Other transcription factors such as myogenin and possibly NR4A1 allow the cells to become myocytes. These cells fuse into multinucleated myotubes that will make up the muscle fibers. 
differentiation of skeletal muscle cells, most likely by transcriptionally activating genes involved in myogenesis. 


\section{LIST OF REFERENCES}

1. Tata, J.R., One hundred years of hormones. EMBO Rep, 2005. 6(6): p. 490-6.

2. Hollenberg, S.M., et al., Primary structure and expression of a functional human glucocorticoid receptor cDNA. Nature, 1985. 318(6047): p. 635-41.

3. Green, S., et al., Cloning of the human oestrogen receptor $c D N A$. J Steroid Biochem, 1986. 24(1): p. 77-83.

4. Jensen, E., A conversation with Elwood Jensen. Interview by David D. Moore. Annu Rev Physiol, 2012. 74: p. 1-11.

5. Kendall, E.C., and Osterberg, A. E, The chemical identification of thyroxin. The Journal of biological chemistry, 1919. 40: p. 265-334.

6. Simoni, R., R. Hill, and M. Vaughan, The Isolation of Thyroxine and Cortisone: the Work of Edward C. Kendall. The Journal of Biological Chemistry, 2002. 277(21): p. e10.

7. Robinson-Rechavi, M., et al., How many nuclear hormone receptors are there in the human genome? Trends Genet, 2001. 17(10): p. 554-6.

8. A unified nomenclature system for the nuclear receptor superfamily. Cell, 1999. 97(2): p. 161-3.

9. Laudet, V., Evolution of the nuclear receptor superfamily: early diversification from an ancestral orphan receptor. J Mol Endocrinol, 1997. 19(3): p. 207-26.

10. Giguere, V., et al., Functional domains of the human glucocorticoid receptor. Cell, 1986. 46(5): p. 645-52.

11. Krust, A., et al., The chicken oestrogen receptor sequence: homology with v-erbA and the human oestrogen and glucocorticoid receptors. EMBO J, 1986. 5(5): p. 891-7.

12. Mi, L.Z., et al., Structural basis for bile acid binding and activation of the nuclear receptor FXR. Mol Cell, 2003. 11(4): p. 1093-100.

13. Miesfeld, R., et al., Genetic complementation of a glucocorticoid receptor deficiency by expression of cloned receptor cDNA. Cell, 1986. 46(3): p. 389-99.

14. Pawlak, M., P. Lefebvre, and B. Staels, General molecular biology and architecture of nuclear receptors. Curr Top Med Chem, 2012. 12(6): p. 486-504.

15. Germain, P., et al., Overview of nomenclature of nuclear receptors. Pharmacol Rev, 2006. 58(4): p. 685-704.

16. Evans, R.M., The steroid and thyroid hormone receptor superfamily. Science, 1988. 240(4854): p. 889-95.

17. Li, Y., et al., Crystallographic identification and functional characterization of phospholipids as ligands for the orphan nuclear receptor steroidogenic factor-1. Mol Cell, 2005. 17(4): p. 491-502.

18. Wang, Z., et al., Structure and function of Nurr1 identifies a class of ligandindependent nuclear receptors. Nature, 2003. 423(6939): p. 555-60.

19. Robinson-Rechavi, M., H. Escriva Garcia, and V. Laudet, The nuclear receptor superfamily. J Cell Sci, 2003. 116(Pt 4): p. 585-6.

20. Ottow, E. and H. Weinmann, Nuclear Receptors as Drug Targets: A Historical Perspective of Modern Drug Discovery, in Nuclear Receptors as Drug Targets. 2008, Weinheim, Germany: Wiley-VCH Verlag GmbH \& Co. KGaA. 
21. Sonoda, J., L. Pei, and R.M. Evans, Nuclear receptors: decoding metabolic disease. FEBS Lett, 2008. 582(1): p. 2-9.

22. Chen, T., Nuclear receptor drug discovery. Curr Opin Chem Biol, 2008. 12(4): p. 418-26.

23. Roeder, R.G., The role of general initiation factors in transcription by $R N A$ polymerase II. Trends Biochem Sci, 1996. 21(9): p. 327-35.

24. Chen, D., S.M. Huang, and M.R. Stallcup, Synergistic, p160 coactivatordependent enhancement of estrogen receptor function by CARM1 and p300. $\mathrm{J}$ Biol Chem, 2000. 275(52): p. 40810-6.

25. Vo, N. and R.H. Goodman, CREB-binding protein and 300 in transcriptional regulation. J Biol Chem, 2001. 276(17): p. 13505-8.

26. McKenna, N.J. and B.W. O'Malley, Combinatorial control of gene expression by nuclear receptors and coregulators. Cell, 2002. 108(4): p. 465-74.

27. Chen, J.D. and R.M. Evans, A transcriptional co-repressor that interacts with nuclear hormone receptors. Nature, 1995. 377(6548): p. 454-7.

28. Horlein, A.J., et al., Ligand-independent repression by the thyroid hormone receptor mediated by a nuclear receptor co-repressor. Nature, 1995. 377(6548): p. 397-404.

29. Ordentlich, P., et al., Unique forms of human and mouse nuclear receptor corepressor SMRT. Proc Natl Acad Sci U S A, 1999. 96(6): p. 2639-44.

30. Park, E.J., et al., SMRTe, a silencing mediator for retinoid and thyroid hormone receptors-extended isoform that is more related to the nuclear receptor corepressor. Proc Natl Acad Sci U S A, 1999. 96(7): p. 3519-24.

31. Gottlicher, M., S. Heck, and P. Herrlich, Transcriptional cross-talk, the second mode of steroid hormone receptor action. J Mol Med (Berl), 1998. 76(7): p. 4809.

32. Shaulian, E. and M. Karin, AP-1 as a regulator of cell life and death. Nat Cell Biol, 2002. 4(5): p. E131-6.

33. Maxwell, M.A. and G.E. Muscat, The NR4A subgroup: immediate early response genes with pleiotropic physiological roles. Nucl Recept Signal, 2006. 4: p. e002.

34. Kouzarides, T., Acetylation: a regulatory modification to rival phosphorylation? EMBO J, 2000. 19(6): p. 1176-9.

35. Wang, C., et al., Direct acetylation of the estrogen receptor alpha hinge region by p300 regulates transactivation and hormone sensitivity. J Biol Chem, 2001. 276(21): p. 18375-83.

36. Fu, M., et al., Androgen receptor acetylation governs trans activation and MEKK1-induced apoptosis without affecting in vitro sumoylation and transrepression function. Mol Cell Biol, 2002. 22(10): p. 3373-88.

37. Perissi, V. and M.G. Rosenfeld, Controlling nuclear receptors: the circular logic of cofactor cycles. Nat Rev Mol Cell Biol, 2005. 6(7): p. 542-54.

38. Overington, J.P., B. Al-Lazikani, and A.L. Hopkins, How many drug targets are there? Nat Rev Drug Discov, 2006. 5(12): p. 993-6.

39. Smith, C.L. and B.W. O'Malley, Coregulator function: a key to understanding tissue specificity of selective receptor modulators. Endocr Rev, 2004. 25(1): p. 45-71. 
40. Smith, C.L., Z. Nawaz, and B.W. O'Malley, Coactivator and corepressor regulation of the agonist/antagonist activity of the mixed antiestrogen, 4hydroxytamoxifen. Mol Endocrinol, 1997. 11(6): p. 657-66.

41. Liu, Z., et al., Coactivator/corepressor ratios modulate PR-mediated transcription by the selective receptor modulator RU486. Proc Natl Acad Sci U S A, 2002. 99(12): p. 7940-4.

42. Webb, P., P. Nguyen, and P.J. Kushner, Differential SERM effects on corepressor binding dictate ERalpha activity in vivo. J Biol Chem, 2003. 278(9): p. 6912-20.

43. Ismail, S.M., The effects of tamoxifen on the uterus. Curr Opin Obstet Gynecol, 1996. 8(1): p. 27-31.

44. Berry, M., D. Metzger, and P. Chambon, Role of the two activating domains of the oestrogen receptor in the cell-type and promoter-context dependent agonistic activity of the anti-oestrogen 4-hydroxytamoxifen. EMBO J, 1990. 9(9): p. 28118.

45. Metzger, D., et al., Promoter specificity of the two transcriptional activation functions of the human oestrogen receptor in yeast. Nucleic Acids Res, 1992. 20(11): p. 2813-7.

46. McInerney, E.M. and B.S. Katzenellenbogen, Different regions in activation function-1 of the human estrogen receptor required for antiestrogen- and estradiol-dependent transcription activation. J Biol Chem, 1996. 271(39): p. 24172-8.

47. Webb, P., et al., Estrogen receptor activation function 1 works by binding 160 coactivator proteins. Mol Endocrinol, 1998. 12(10): p. 1605-18.

48. Shang, Y., et al., Cofactor dynamics and sufficiency in estrogen receptorregulated transcription. Cell, 2000. 103(6): p. 843-52.

49. Love, R.R., et al., Effects of tamoxifen on bone mineral density in postmenopausal women with breast cancer. N Engl J Med, 1992. 326(13): p. 852-6.

50. Delmas, P.D., et al., Effects of raloxifene on bone mineral density, serum cholesterol concentrations, and uterine endometrium in postmenopausal women. N Engl J Med, 1997. 337(23): p. 1641-7.

51. Turner, C.H., M. Sato, and H.U. Bryant, Raloxifene preserves bone strength and bone mass in ovariectomized rats. Endocrinology, 1994. 135(5): p. 2001-5.

52. McKay, L.I. and J.A. Cidlowski, Molecular control of immune/inflammatory responses: interactions between nuclear factor-kappa $B$ and steroid receptorsignaling pathways. Endocr Rev, 1999. 20(4): p. 435-59.

53. Newton, R. and N.S. Holden, Separating transrepression and transactivation: $a$ distressing divorce for the glucocorticoid receptor? Mol Pharmacol, 2007. 72(4): p. 799-809.

54. Schacke, H., et al., Selective glucocorticoid receptor agonists (SEGRAs): novel ligands with an improved therapeutic index. Mol Cell Endocrinol, 2007. 275(1-2): p. 109-17.

55. Miner, J.N., M.H. Hong, and A. Negro-Vilar, New and improved glucocorticoid receptor ligands. Expert Opin Investig Drugs, 2005. 14(12): p. 1527-45.

56. Lehmann, J.M., et al., An antidiabetic thiazolidinedione is a high affinity ligand for peroxisome proliferator-activated receptor gamma (PPAR gamma). J Biol Chem, 1995. 270(22): p. 12953-6. 
57. Harris, K.A. and D.M. Reese, Treatment options in hormone-refractory prostate cancer: current and future approaches. Drugs, 2001. 61(15): p. 2177-92.

58. Santos, A.F., H. Huang, and D.J. Tindall, The androgen receptor: a potential target for therapy of prostate cancer. Steroids, 2004. 69(2): p. 79-85.

59. Moore, J.T., J.L. Collins, and K.H. Pearce, The nuclear receptor superfamily and drug discovery. ChemMedChem, 2006. 1(5): p. 504-23.

60. Gao, W. and J.T. Dalton, Expanding the therapeutic use of androgens via selective androgen receptor modulators (SARMs). Drug Discov Today, 2007. 12(5-6): p. 241-8.

61. Evans, R.M. and D.J. Mangelsdorf, Nuclear Receptors, RXR, and the Big Bang. Cell, 2014. 157(1): p. 255-66.

62. Wipf, P., et al., New opportunities for pregnane X receptor $(P X R)$ targeting in drug development. lessons from Enantio- and species-specific PXR ligands identified from a discovery library of amino acid analogues. Mini Rev Med Chem, 2007. 7(6): p. 617-25.

63. Sodhi, R.K. and N. Singh, Liver X receptors: emerging therapeutic targets for Alzheimer's disease. Pharmacol Res, 2013. 72: p. 45-51.

64. Zollner, G. and M. Trauner, Nuclear receptors as therapeutic targets in cholestatic liver diseases. Br J Pharmacol, 2009. 156(1): p. 7-27.

65. Yang, C., Q. Li, and Y. Li, Targeting nuclear receptors with marine natural products. Mar Drugs, 2014. 12(2): p. 601-35.

66. Milbrandt, J., Nerve growth factor induces a gene homologous to the glucocorticoid receptor gene. Neuron, 1988. 1(3): p. 183-8.

67. Safe, S., et al., NR4A orphan receptors and cancer. Nuclear Receptor Signaling, 2011. 9.

68. Chang, C., et al., Isolation and characterization of human TR3 receptor: a member of steroid receptor superfamily. J Steroid Biochem, 1989. 34(1-6): p. 391-5.

69. Law, S.W., et al., Identification of a new brain-specific transcription factor, NURR1. Mol Endocrinol, 1992. 6(12): p. 2129-35.

70. Saucedo-Cardenas, O., et al., Cloning and structural organization of the gene encoding the murine nuclear receptor transcription factor, NURR1. Gene, 1997. 187(1): p. 135-9.

71. Castillo, S.O., et al., Organization, sequence, chromosomal localization, and promoter identification of the mouse orphan nuclear receptor Nurrl gene. Genomics, 1997. 41(2): p. 250-7.

72. Ichinose, H., et al., Molecular cloning of the human Nurr1 gene: characterization of the human gene and cDNAs. Gene, 1999. 230(2): p. 233-9.

73. Ohkura, N., et al., Molecular cloning of a novel thyroid/steroid receptor superfamily gene from cultured rat neuronal cells. Biochem Biophys Res Commun, 1994. 205(3): p. 1959-65.

74. Safe, S., et al., Nuclear receptor $4 A$ (NR4A) family - orphans no more. J Steroid Biochem Mol Biol, 2016. 157: p. 48-60.

75. Vinayavekhin, N. and A. Saghatelian, Discovery of a protein-metabolite interaction between unsaturated fatty acids and the nuclear receptor Nur77 using a metabolomics approach. J Am Chem Soc, 2011. 133(43): p. 17168-71. 
76. de Vera, I.M., et al., Identification of a Binding Site for Unsaturated Fatty Acids in the Orphan Nuclear Receptor Nurr1. ACS Chem Biol, 2016.

77. Wansa, K.D., J.M. Harris, and G.E. Muscat, The activation function-1 domain of Nur77/NR4A1 mediates trans-activation, cell specificity, and coactivator recruitment. J Biol Chem, 2002. 277(36): p. 33001-11.

78. Wansa, K.D., et al., The AF-1 domain of the orphan nuclear receptor NOR-1 mediates trans-activation, coactivator recruitment, and activation by the purine anti-metabolite 6-mercaptopurine. J Biol Chem, 2003. 278(27): p. 24776-90.

79. Baker, K.D., et al., The Drosophila orphan nuclear receptor DHR38 mediates an atypical ecdysteroid signaling pathway. Cell, 2003. 113(6): p. 731-42.

80. Murphy, E.P., et al., Differential regulation of transcription by the NURR1/NUR77 subfamily of nuclear transcription factors. Gene Expr, 1996. 5(3): p. 169-79.

81. Paulsen, R.F., et al., Three related brain nuclear receptors, NGFI-B, Nurr1, and NOR-1, as transcriptional activators. J Mol Neurosci, 1995. 6(4): p. 249-55.

82. Codina, A., et al., Identification of a novel co-regulator interaction surface on the ligand binding domain of Nurr1 using NMR footprinting. J Biol Chem, 2004. 279(51): p. 53338-45.

83. Flaig, R., et al., Structural basis for the cell-specific activities of the NGFI-B and the Nurrl ligand-binding domain. J Biol Chem, 2005. 280(19): p. 19250-8.

84. Castro, D.S., et al., Activity of the Nurr 1 carboxyl-terminal domain depends on cell type and integrity of the activation function 2. J Biol Chem, 1999. 274(52): p. 37483-90.

85. Maira, M., et al., Dimer-specific potentiation of NGFI-B (Nur77) transcriptional activity by the protein kinase A pathway and AF-1-dependent coactivator recruitment. Mol Cell Biol, 2003. 23(3): p. 763-76.

86. Davis, I.J., et al., Functional domains and phosphorylation of the orphan receptor Nur77. Mol Endocrinol, 1993. 7(8): p. 953-64.

87. Maltais, A. and Y. Labelle, Structure and expression of the mouse gene encoding the orphan nuclear receptor TEC. DNA Cell Biol, 2000. 19(2): p. 121-30.

88. Paulsen, R.E., et al., Domains regulating transcriptional activity of the inducible orphan receptor NGFI-B. J Biol Chem, 1992. 267(23): p. 16491-6.

89. Maira, M., et al., Heterodimerization between members of the Nur subfamily of orphan nuclear receptors as a novel mechanism for gene activation. Mol Cell Biol, 1999. 19(11): p. 7549-57.

90. Philips, A., et al., Novel dimeric Nur77 signaling mechanism in endocrine and lymphoid cells. Mol Cell Biol, 1997. 17(10): p. 5946-51.

91. Perlmann, T. and L. Jansson, A novel pathway for vitamin A signaling mediated by RXR heterodimerization with NGFI-B and NURR1. Genes Dev, 1995. 9(7): p. 769-82.

92. Zetterstrom, R.H., et al., Retinoid X receptor heterodimerization and developmental expression distinguish the orphan nuclear receptors NGFI-B, Nurr1, and Nor1. Mol Endocrinol, 1996. 10(12): p. 1656-66.

93. Wilson, T.E., et al., In vivo mutational analysis of the NGFI-A zinc fingers. J Biol Chem, 1992. 267(6): p. 3718-24. 
94. Wilson, T.E., et al., Identification of the DNA binding site for NGFI-B by genetic selection in yeast. Science, 1991. 252(5010): p. 1296-300.

95. Wilson, T.E., et al., The orphan nuclear receptor NGFI-B regulates expression of the gene encoding steroid 21-hydroxylase. Mol Cell Biol, 1993. 13(2): p. 861-8.

96. Zhao, Y. and D. Bruemmer, NR4A orphan nuclear receptors: transcriptional regulators of gene expression in metabolism and vascular biology. Arterioscler Thromb Vasc Biol, 2010. 30(8): p. 1535-41.

97. Mohan, H.M., et al., Molecular pathways: the role of NR4A orphan nuclear receptors in cancer. Clin Cancer Res, 2012. 18(12): p. 3223-8.

98. Borghaei, R.C., et al., Induction of mitogen-inducible nuclear orphan receptor by interleukin 1 in human synovial and gingival fibroblasts. Biochem Biophys Res Commun, 1998. 251(1): p. 334-8.

99. Fahrner, T.J., S.L. Carroll, and J. Milbrandt, The NGFI-B protein, an inducible member of the thyroid/steroid receptor family, is rapidly modified posttranslationally. Mol Cell Biol, 1990. 10(12): p. 6454-9.

100. Hazel, T.G., D. Nathans, and L.F. Lau, A gene inducible by serum growth factors encodes a member of the steroid and thyroid hormone receptor superfamily. Proc Natl Acad Sci U S A, 1988. 85(22): p. 8444-8.

101. Honkaniemi, J., et al., Induction of multiple immediate early genes in rat hypothalamic paraventricular nucleus after stress. Brain Res Mol Brain Res, 1994. 25(3-4): p. 234-41.

102. Honkaniemi, J., et al., Stress induces zinc finger immediate early genes in the rat adrenal gland. Brain Res, 2000. 877(2): p. 203-8.

103. Kagaya, S., et al., Prostaglandin A2 acts as a transactivator for NOR1 (NR4A3) within the nuclear receptor superfamily. Biol Pharm Bull, 2005. 28(9): p. 1603-7.

104. Katagiri, Y., et al., Differential regulation of the transcriptional activity of the orphan nuclear receptor NGFI-B by membrane depolarization and nerve growth factor. J Biol Chem, 1997. 272(50): p. 31278-84.

105. Roche, E., et al., Palmitate and oleate induce the immediate-early response genes c-fos and nur-77 in the pancreatic beta-cell line INS-1. Diabetes, 1999. 48(10): p. 2007-14.

106. Tetradis, S., O. Bezouglaia, and A. Tsingotjidou, Parathyroid hormone induces expression of the nuclear orphan receptor Nurr1 in bone cells. Endocrinology, 2001. 142(2): p. 663-70.

107. Tetradis, S., et al., Regulation of the nuclear orphan receptor Nur77 in bone by parathyroid hormone. Biochem Biophys Res Commun, 2001. 281(4): p. 913-6.

108. Tippetts, M.T., et al., Tumor promoter-inducible genes are differentially expressed in the developing mouse. Mol Cell Biol, 1988. 8(10): p. 4570-2.

109. Williams, G.T. and L.F. Lau, Activation of the inducible orphan receptor gene nur77 by serum growth factors: dissociation of immediate-early and delayedearly responses. Mol Cell Biol, 1993. 13(10): p. 6124-36.

110. Bandoh, S., et al., Mechanical agitation induces gene expression of NOR-1 and its closely related orphan nuclear receptors in leukemic cell lines. Leukemia, 1997. 11(9): p. 1453-8. 
111. Hazel, T.G., et al., Nur77 is differentially modified in PC12 cells upon membrane depolarization and growth factor treatment. Mol Cell Biol, 1991. 11(6): p. 323946.

112. Miyakoshi, J., et al., Enhanced NOR-1 gene expression by exposure of Chinese hamster cells to high-density $50 \mathrm{~Hz}$ magnetic fields. Mol Cell Biochem, 1998. 181(1-2): p. 191-5.

113. Arkenbout, E.K., et al., Protective function of transcription factor TR3 orphan receptor in atherogenesis: decreased lesion formation in carotid artery ligation model in TR3 transgenic mice. Circulation, 2002. 106(12): p. 1530-5.

114. Lim, R.W., W.L. Yang, and H. Yu, Signal-transduction-pathway-specific desensitization of expression of orphan nuclear receptor TIS1. Biochem J, 1995. 308 ( Pt 3): p. 785-9.

115. Nakai, A., et al., A human early response gene homologous to murine nur77 and rat NGFI-B, and related to the nuclear receptor superfamily. Mol Endocrinol, 1990. 4(10): p. 1438-43.

116. Winoto, A., Genes involved in T-cell receptor-mediated apoptosis of thymocytes and T-cell hybridomas. Semin Immunol, 1997. 9(1): p. 51-8.

117. Liu, Z.G., et al., Apoptotic signals delivered through the T-cell receptor of a $T$ cell hybrid require the immediate-early gene nur77. Nature, 1994. 367(6460): p. 281-4.

118. Woronicz, J.D., et al., Requirement for the orphan steroid receptor Nur77 in apoptosis of T-cell hybridomas. Nature, 1994. 367(6460): p. 277-81.

119. Calnan, B.J., et al., A role for the orphan steroid receptor Nur77 in apoptosis accompanying antigen-induced negative selection. Immunity, 1995. 3(3): p. 27382.

120. Zhou, T., et al., Inhibition of Nur77/Nurrl leads to inefficient clonal deletion of self-reactive T cells. J Exp Med, 1996. 183(4): p. 1879-92.

121. Masuyama, N., et al., Akt inhibits the orphan nuclear receptor Nur77 and T-cell apoptosis. J Biol Chem, 2001. 276(35): p. 32799-805.

122. Weih, F., et al., Apoptosis of nur77/N10-transgenic thymocytes involves the Fas/Fas ligand pathway. Proc Natl Acad Sci U S A, 1996. 93(11): p. 5533-8.

123. Thompson, J. and A. Winoto, During negative selection, Nur77 family proteins translocate to mitochondria where they associate with Bcl-2 and expose its proapoptotic BH3 domain. J Exp Med, 2008. 205(5): p. 1029-36.

124. Woronicz, J.D., et al., Regulation of the Nur77 orphan steroid receptor in activation-induced apoptosis. Mol Cell Biol, 1995. 15(11): p. 6364-76.

125. Sakurada, K., et al., Nurr1, an orphan nuclear receptor, is a transcriptional activator of endogenous tyrosine hydroxylase in neural progenitor cells derived from the adult brain. Development, 1999. 126(18): p. 4017-26.

126. Zetterstrom, R.H., et al., Dopamine neuron agenesis in Nurrl-deficient mice. Science, 1997. 276(5310): p. 248-50.

127. Le, W.D., et al., Mutations in NR4A2 associated with familial Parkinson disease. Nat Genet, 2003. 33(1): p. 85-9.

128. Hawk, J.D., et al., NR4A nuclear receptors support memory enhancement by histone deacetylase inhibitors. J Clin Invest, 2012. 122(10): p. 3593-602. 
129. McNulty, S.E., et al., Differential roles for Nr4al and Nr4a2 in object location vs. object recognition long-term memory. Learn Mem, 2012. 19(12): p. 588-92.

130. Mahmoudi, S., P.J. Blanchet, and D. Levesque, Haloperidol-induced striatal Nur77 expression in a non-human primate model of tardive dyskinesia. Eur J Neurosci, 2013. 38(1): p. 2192-8.

131. Levesque, D. and C. Rouillard, Nur77 and retinoid X receptors: crucial factors in dopamine-related neuroadaptation. Trends Neurosci, 2007. 30(1): p. 22-30.

132. Xiao, G., et al., NR4A1 enhances neural survival following oxygen and glucose deprivation: an in vitro study. J Neurol Sci, 2013. 330(1-2): p. 78-84.

133. Crawford, P.A., et al., Adrenocortical function and regulation of the steroid 21hydroxylase gene in NGFI-B-deficient mice. Mol Cell Biol, 1995. 15(8): p. 433116.

134. Fernandez, P.M., et al., Nuclear receptors Norl and NGFI-B/Nur77 play similar, albeit distinct, roles in the hypothalamo-pituitary-adrenal axis. Endocrinology, 2000. 141(7): p. 2392-400.

135. Murphy, E.P., et al., Involvement of the nuclear orphan receptor NURR1 in the regulation of corticotropin-releasing hormone expression and actions in human inflammatory arthritis. Arthritis Rheum, 2001. 44(4): p. 782-93.

136. Barish, G.D., et al., A Nuclear Receptor Atlas: macrophage activation. Mol Endocrinol, 2005. 19(10): p. 2466-77.

137. Pei, L., et al., Induction of NR4A orphan nuclear receptor expression in macrophages in response to inflammatory stimuli. J Biol Chem, 2005. 280(32): p. 29256-62.

138. Sekiya, T., et al., Nr4a receptors are essential for thymic regulatory $T$ cell development and immune homeostasis. Nat Immunol, 2013. 14(3): p. 230-7.

139. Fassett, M.S., et al., Nuclear receptor Nr4al modulates both regulatory T-cell (Treg) differentiation and clonal deletion. Proc Natl Acad Sci U S A, 2012. 109(10): p. 3891-6.

140. Ipseiz, N., et al., The nuclear receptor Nr4al mediates anti-inflammatory effects of apoptotic cells. J Immunol, 2014. 192(10): p. 4852-8.

141. Mahoney, D.J., et al., Analysis of global mRNA expression in human skeletal muscle during recovery from endurance exercise. FASEB J, 2005. 19(11): p. 1498-500.

142. Maxwell, M.A., et al., Nur77 regulates lipolysis in skeletal muscle cells. Evidence for cross-talk between the beta-adrenergic and an orphan nuclear hormone receptor pathway. J Biol Chem, 2005. 280(13): p. 12573-84.

143. Fu, M., et al., A Nuclear Receptor Atlas: 3T3-L1 adipogenesis. Mol Endocrinol, 2005. 19(10): p. 2437-50.

144. Pei, L., et al., NR4A orphan nuclear receptors are transcriptional regulators of hepatic glucose metabolism. Nat Med, 2006. 12(9): p. 1048-55.

145. Berriel Diaz, M., U. Lemke, and S. Herzig, Discovering orphans' sweet secret: NR4A receptors and hepatic glucose production. Cell Metab, 2006. 4(5): p. 33940.

146. Pols, T.W., et al., Nur77 modulates hepatic lipid metabolism through suppression of SREBP Ic activity. Biochem Biophys Res Commun, 2008. 366(4): p. 910-6. 
147. Chao, L.C., et al., Insulin resistance and altered systemic glucose metabolism in mice lacking Nur77. Diabetes, 2009. 58(12): p. 2788-96.

148. Chao, L.C., et al., Nur77 coordinately regulates expression of genes linked to glucose metabolism in skeletal muscle. Mol Endocrinol, 2007. 21(9): p. 2152-63.

149. Kanzleiter, T., et al., Evidence for Nr4al as a cold-induced effector of brown fat thermogenesis. Physiol Genomics, 2005. 24(1): p. 37-44.

150. Hu, Y.W., et al., Nur77 decreases atherosclerosis progression in apoE(-/-) mice fed a high-fat/high-cholesterol diet. PLoS One, 2014. 9(1): p. e87313.

151. Hanna, R.N., et al., NR4A1 (Nur77) deletion polarizes macrophages toward an inflammatory phenotype and increases atherosclerosis. Circ Res, 2012. 110(3): p. 416-27.

152. Hilgendorf, I., et al., Ly-6Chigh monocytes depend on Nr4al to balance both inflammatory and reparative phases in the infarcted myocardium. Circ Res, 2014. 114(10): p. 1611-22.

153. Zhao, Y., et al., Deficiency of the NR4A orphan nuclear receptor NORI decreases monocyte adhesion and atherosclerosis. Circ Res, 2010. 107(4): p. 501-11.

154. Lee, S.L., et al., Unimpaired thymic and peripheral T cell death in mice lacking the nuclear receptor NGFI-B (Nur77). Science, 1995. 269(5223): p. 532-5.

155. Cheng, L.E., et al., Functional redundancy of the Nur77 and Nor-1 orphan steroid receptors in T-cell apoptosis. EMBO J, 1997. 16(8): p. 1865-75.

156. Mullican, S.E., et al., Abrogation of nuclear receptors Nr4a3 and Nr4a1 leads to development of acute myeloid leukemia. Nat Med, 2007. 13(6): p. 730-5.

157. Castillo, S.O., et al., A divergent role of COOH-terminal domains in Nurrl and Nur77 transactivation. Gene Expr, 1998. 7(1): p. 1-12.

158. Nsegbe, E., et al., Congenital hypoventilation and impaired hypoxic response in Nurr1 mutant mice. J Physiol, 2004. 556(Pt 1): p. 43-59.

159. Ponnio, T., et al., The nuclear receptor Nor-1 is essential for proliferation of the semicircular canals of the mouse inner ear. Mol Cell Biol, 2002. 22(3): p. 935-45.

160. Ponnio, T. and O.M. Conneely, nor-1 regulates hippocampal axon guidance, pyramidal cell survival, and seizure susceptibility. Mol Cell Biol, 2004. 24(20): p. 9070-8.

161. DeYoung, R.A., et al., The orphan steroid receptor Nur77 family member Nor-1 is essential for early mouse embryogenesis. J Biol Chem, 2003. 278(47): p. 47104-9.

162. Cao, X., et al., Retinoid X receptor regulates Nur77/TR3-dependent apoptosis [corrected] by modulating its nuclear export and mitochondrial targeting. Mol Cell Biol, 2004. 24(22): p. 9705-25.

163. Dawson, M.I., et al., Apoptosis induction in cancer cells by a novel analogue of 6[3-(1-adamantyl)-4-hydroxyphenyl]-2-naphthalenecarboxylic acid lacking retinoid receptor transcriptional activation activity. Cancer Res, 2001. 61(12): p. 4723-30.

164. Holmes, W.F., D.R. Soprano, and K.J. Soprano, Comparison of the mechanism of induction of apoptosis in ovarian carcinoma cells by the conformationally restricted synthetic retinoids CD437 and 4-HPR. J Cell Biochem, 2003. 89(2): p. 262-78. 
165. Holmes, W.F., D.R. Soprano, and K.J. Soprano, Early events in the induction of apoptosis in ovarian carcinoma cells by CD437: activation of the p38 MAP kinase signal pathway. Oncogene, 2003. 22(41): p. 6377-86.

166. Holmes, W.F., D.R. Soprano, and K.J. Soprano, Synthetic retinoids as inducers of apoptosis in ovarian carcinoma cell lines. J Cell Physiol, 2004. 199(3): p. 317-29.

167. Kolluri, S.K., et al., Mitogenic effect of orphan receptor TR3 and its regulation by MEKK1 in lung cancer cells. Mol Cell Biol, 2003. 23(23): p. 8651-67.

168. Li, H., et al., Cytochrome c release and apoptosis induced by mitochondrial targeting of nuclear orphan receptor TR3. Science, 2000. 289(5482): p. 1159-64.

169. Li, Y., et al., Molecular determinants of AHPN (CD437)-induced growth arrest and apoptosis in human lung cancer cell lines. Mol Cell Biol, 1998. 18(8): p. 4719-31.

170. Lin, X.F., et al., RXRalpha acts as a carrier for TR3 nuclear export in a 9-cis retinoic acid-dependent manner in gastric cancer cells. J Cell Sci, 2004. 117(Pt 23): p. 5609-21.

171. Zhan, Y., et al., Cytosporone B is an agonist for nuclear orphan receptor Nur77. Nat Chem Biol, 2008. 4(9): p. 548-56.

172. To, S.K., J.Z. Zeng, and A.S. Wong, Nur77: a potential therapeutic target in cancer. Expert Opin Ther Targets, 2012. 16(6): p. 573-85.

173. Liu, J.J., et al., A unique pharmacophore for activation of the nuclear orphan receptor Nur77 in vivo and in vitro. Cancer Res, 2010. 70(9): p. 3628-37.

174. Lee, S.O., et al., Targeting NR4A1 (TR3) in cancer cells and tumors. Expert Opin Ther Targets, 2011. 15(2): p. 195-206.

175. Lin, B., et al., Conversion of Bcl-2 from protector to killer by interaction with nuclear orphan receptor Nur77/TR3. Cell, 2004. 116(4): p. 527-40.

176. Chen, Y.L., et al., The induction of orphan nuclear receptor Nur77 expression by n-butylenephthalide as pharmaceuticals on hepatocellular carcinoma cell therapy. Mol Pharmacol, 2008. 74(4): p. 1046-58.

177. Gennari, A., et al., Identification by DNA macroarray of nur77 as a gene induced by di-n-butyltin dichloride: its role in organotin-induced apoptosis. Toxicol Appl Pharmacol, 2002. 181(1): p. 27-31.

178. Jeong, J.H., et al., Orphan nuclear receptor Nur77 translocates to mitochondria in the early phase of apoptosis induced by synthetic chenodeoxycholic acid derivatives in human stomach cancer cell line SNU-1. Ann N Y Acad Sci, 2003. 1010: p. 171-7.

179. Wilson, A.J., et al., TR3 modulates platinum resistance in ovarian cancer. Cancer Res, 2013. 73(15): p. 4758-69.

180. Wilson, A.J., et al., TR3/Nur77 in colon cancer cell apoptosis. Cancer Res, 2003. 63(17): p. 5401-7.

181. Liu, S., et al., Induction of apoptosis by TPA and VP-16 is through translocation of TR3. World J Gastroenterol, 2002. 8(3): p. 446-50.

182. Lee, J.M., et al., Epstein-Barr virus EBNA2 blocks Nur77-mediated apoptosis. Proc Natl Acad Sci U S A, 2002. 99(18): p. 11878-83.

183. Lee, K.W., et al., Rapid apoptosis induction by IGFBP-3 involves an insulin-like growth factor-independent nucleomitochondrial translocation of RXRalpha/Nur77. J Biol Chem, 2005. 280(17): p. 16942-8. 
184. Liu, J., et al., Modulation of orphan nuclear receptor Nur77-mediated apoptotic pathway by acetylshikonin and analogues. Cancer Res, 2008. 68(21): p. 8871-80.

185. Shin, H.J., et al., Induction of orphan nuclear receptor Nur77 gene expression and its role in cadmium-induced apoptosis in lung. Carcinogenesis, 2004. 25(8): p. 1467-75.

186. Liang, B., et al., Involvement of TR3/Nur77 translocation to the endoplasmic reticulum in ER stress-induced apoptosis. Exp Cell Res, 2007. 313(13): p. 283344.

187. Chintharlapalli, S., et al., Activation of Nur77 by selected 1,1-Bis(3'-indolyl)-1-(psubstituted phenyl)methanes induces apoptosis through nuclear pathways. J Biol Chem, 2005. 280(26): p. 24903-14.

188. Cho, S.D., et al., Nur77 agonists induce proapoptotic genes and responses in colon cancer cells through nuclear receptor-dependent and nuclear receptorindependent pathways. Cancer Res, 2007. 67(2): p. 674-83.

189. Kolluri, S.K., et al., A short Nur77-derived peptide converts Bcl-2 from a protector to a killer. Cancer Cell, 2008. 14(4): p. 285-98.

190. Ferlini, C., et al., Paclitaxel directly binds to Bcl-2 and functionally mimics activity of Nur77. Cancer Res, 2009. 69(17): p. 6906-14.

191. Lee, R.C., R.L. Feinbaum, and V. Ambros, The C. elegans heterochronic gene lin-4 encodes small RNAs with antisense complementarity to lin-14. Cell, 1993. 75(5): p. 843-54.

192. Reinhart, B.J., et al., The 21-nucleotide let-7 RNA regulates developmental timing in Caenorhabditis elegans. Nature, 2000. 403(6772): p. 901-6.

193. Slack, F.J., et al., The lin-41 RBCC gene acts in the C. elegans heterochronic pathway between the let-7 regulatory RNA and the LIN-29 transcription factor. Mol Cell, 2000. 5(4): p. 659-69.

194. Pasquinelli, A.E., et al., Conservation of the sequence and temporal expression of let-7 heterochronic regulatory RNA. Nature, 2000. 408(6808): p. 86-9.

195. Bhaskaran, M. and M. Mohan, MicroRNAs: history, biogenesis, and their evolving role in animal development and disease. Vet Pathol, 2014. 51(4): p. 75974.

196. Ambros, V., et al., A uniform system for microRNA annotation. RNA, 2003. 9(3): p. $277-9$.

197. Griffiths-Jones, S., The microRNA Registry. Nucleic Acids Res, 2004. 32(Database issue): p. D109-11.

198. Griffiths-Jones, S., et al., miRBase: microRNA sequences, targets and gene nomenclature. Nucleic Acids Res, 2006. 34(Database issue): p. D140-4.

199. Griffiths-Jones, S., et al., miRBase: tools for microRNA genomics. Nucleic Acids Res, 2008. 36(Database issue): p. D154-8.

200. Hutvagner, G. and P.D. Zamore, A microRNA in a multiple-turnover RNAi enzyme complex. Science, 2002. 297(5589): p. 2056-60.

201. Guo, H., et al., Mammalian microRNAs predominantly act to decrease target $m R N A$ levels. Nature, 2010. 466(7308): p. 835-40.

202. Saini, H.K., S. Griffiths-Jones, and A.J. Enright, Genomic analysis of human microRNA transcripts. Proc Natl Acad Sci U S A, 2007. 104(45): p. 17719-24. 
203. Lee, Y., et al., The nuclear RNase III Drosha initiates microRNA processing. Nature, 2003. 425(6956): p. 415-9.

204. Lund, E., et al., Nuclear export of microRNA precursors. Science, 2004. 303(5654): p. 95-8.

205. Yi, R., et al., Exportin-5 mediates the nuclear export of pre-microRNAs and short hairpin RNAs. Genes Dev, 2003. 17(24): p. 3011-6.

206. Chendrimada, T.P., et al., TRBP recruits the Dicer complex to Ago2 for microRNA processing and gene silencing. Nature, 2005. 436(7051): p. 740-4.

207. Bartel, D.P., MicroRNAs: genomics, biogenesis, mechanism, and function. Cell, 2004. 116(2): p. 281-97.

208. Okamura, K., N. Liu, and E.C. Lai, Distinct mechanisms for microRNA strand selection by Drosophila Argonautes. Mol Cell, 2009. 36(3): p. 431-44.

209. Lytle, J.R., T.A. Yario, and J.A. Steitz, Target $m R N A$ s are repressed as efficiently by microRNA-binding sites in the 5' UTR as in the 3' UTR. Proc Natl Acad Sci U S A, 2007. 104(23): p. 9667-72.

210. Tay, Y., et al., MicroRNAs to Nanog, Oct4 and Sox2 coding regions modulate embryonic stem cell differentiation. Nature, 2008. 455(7216): p. 1124-8.

211. Friedman, R.C., et al., Most mammalian mRNAs are conserved targets of microRNAs. Genome Res, 2009. 19(1): p. 92-105.

212. Esquela-Kerscher, A. and F.J. Slack, Oncomirs - microRNAs with a role in cancer. Nat Rev Cancer, 2006. 6(4): p. 259-69.

213. Hammond, S.M., MicroRNAs as tumor suppressors. Nat Genet, 2007. 39(5): p. 582-3.

214. Sandoval, J. and M. Esteller, Cancer epigenomics: beyond genomics. Curr Opin Genet Dev, 2012. 22(1): p. 50-5.

215. Bader, A.G., miR-34 - a microRNA replacement therapy is headed to the clinic. Front Genet, 2012. 3: p. 120.

216. Yamakuchi, M., M. Ferlito, and C.J. Lowenstein, miR-34a repression of SIRTI regulates apoptosis. Proc Natl Acad Sci U S A, 2008. 105(36): p. 13421-6.

217. Bommer, G.T., et al., p53-mediated activation of miRNA34 candidate tumorsuppressor genes. Curr Biol, 2007. 17(15): p. 1298-307.

218. Schickel, R., et al., MicroRNAs: key players in the immune system, differentiation, tumorigenesis and cell death. Oncogene, 2008. 27(45): p. 5959-74.

219. Orellana, E.A. and A.L. Kasinski, MicroRNAs in Cancer: A Historical Perspective on the Path from Discovery to Therapy. Cancers (Basel), 2015. 7(3): p. 1388-405.

220. Farazi, T.A., et al., miRNAs in human cancer. J Pathol, 2011. 223(2): p. 102-15.

221. Jopling, C., Liver-specific microRNA-122: Biogenesis and function. RNA Biol, 2012. 9(2): p. 137-42.

222. Lanford, R.E., et al., Therapeutic silencing of microRNA-122 in primates with chronic hepatitis $C$ virus infection. Science, 2010. 327(5962): p. 198-201.

223. Inamoto, T., et al., 1,1-Bis(3'-indolyl)-1-(p-chlorophenyl)methane activates the orphan nuclear receptor Nurr1 and inhibits bladder cancer growth. Mol Cancer Ther, 2008. 7(12): p. 3825-33. 
224. Cho, S.D., et al., Activation of nerve growth factor-induced B alpha by methylenesubstituted diindolylmethanes in bladder cancer cells induces apoptosis and inhibits tumor growth. Mol Pharmacol, 2010. 77(3): p. 396-404.

225. Ohkubo, T., et al., Early induction of the orphan nuclear receptor NOR-1 during cell death of the human breast cancer cell line MCF-7. Mol Cell Endocrinol, 2000. 162(1-2): p. 151-6.

226. Ye, X., et al., Distinct role and functional mode of TR3 and RARalpha in mediating ATRA-induced signalling pathway in breast and gastric cancer cells. Int J Biochem Cell Biol, 2004. 36(1): p. 98-113.

227. Alexopoulou, A.N., et al., Dissecting the transcriptional networks underlying breast cancer: NR4A1 reduces the migration of normal and breast cancer cell lines. Breast Cancer Res, 2010. 12(4): p. R51.

228. Holbeck, S., et al., Expression profiling of nuclear receptors in the NCI60 cancer cell panel reveals receptor-drug and receptor-gene interactions. Mol Endocrinol, 2010. 24(6): p. 1287-96.

229. Holla, V.R., et al., Nuclear orphan receptor NR4A2 modulates fatty acid oxidation pathways in colorectal cancer. J Biol Chem, 2011. 286(34): p. 30003-9.

230. Wu, H., et al., Regulation of Nur77 expression by beta-catenin and its mitogenic effect in colon cancer cells. FASEB J, 2011. 25(1): p. 192-205.

231. Hu, Y., et al., Bile acids regulate nuclear receptor (Nur77) expression and intracellular location to control proliferation and apoptosis. Mol Cancer Res, 2015. 13(2): p. 281-92.

232. Zhao, B.X., et al., Orphan receptor TR3 enhances p53 transactivation and represses DNA double-strand break repair in hepatoma cells under ionizing radiation. Mol Endocrinol, 2011. 25(8): p. 1337-50.

233. Lee, S.O., et al., Inactivation of the orphan nuclear receptor TR3/Nur77 inhibits pancreatic cancer cell and tumor growth. Cancer Res, 2010. 70(17): p. 6824-36.

234. Yoon, K., et al., Activation of nuclear TR3 (NR4A1) by a diindolylmethane analog induces apoptosis and proapoptotic genes in pancreatic cancer cells and tumors. Carcinogenesis, 2011. 32(6): p. 836-42.

235. Uemura, H. and C. Chang, Antisense TR3 orphan receptor can increase prostate cancer cell viability with etoposide treatment. Endocrinology, 1998. 139(5): p. 2329-34.

236. Delgado, E., et al., High expression of orphan nuclear receptor NR4A1 in a subset of ovarian tumors with worse outcome. Gynecol Oncol, 2016. 141(2): p. 348-56.

237. Ueda, Y., et al., Expression of nerve growth factor-induced clone B subfamily and pro-opiomelanocortin gene in lung cancer cell lines. Am J Respir Cell Mol Biol, 1999. 20(6): p. 1319-25.

238. Lee, S.O., et al., The nuclear receptor TR3 regulates $m$ TORC1 signaling in lung cancer cells expressing wild-type p53. Oncogene, 2012. 31(27): p. 3265-76.

239. Zeng, H., et al., Orphan nuclear receptor TR3/Nur77 regulates VEGF-A-induced angiogenesis through its transcriptional activity. J Exp Med, 2006. 203(3): p. 719-29.

240. Baek, D., et al., The impact of microRNAs on protein output. Nature, 2008. 455(7209): p. 64-71. 
241. Jeyaseelan, K., K.Y. Lim, and A. Armugam, MicroRNA expression in the blood and brain of rats subjected to transient focal ischemia by middle cerebral artery occlusion. Stroke, 2008. 39(3): p. 959-66.

242. Tenga, A., et al., Regulation of Nuclear Receptor Nur77 by miR-124. PLoS One, 2016. 11(2): p. e0148433.

243. Kang, S.A., et al., Regulation of Nur77 protein turnover through acetylation and deacetylation induced by $\mathrm{p} 300$ and HDAC1. Biochem Pharmacol, 2010. 80(6): $\mathrm{p}$. $867-73$.

244. To, S.K., et al., Hypoxia triggers a Nur77-beta-catenin feed-forward loop to promote the invasive growth of colon cancer cells. Br J Cancer, 2014. 110(4): $\mathrm{p}$. 935-45.

245. Choi, J.W., et al., Nur77 activated by hypoxia-inducible factor-1alpha overproduces proopiomelanocortin in von Hippel-Lindau-mutated renal cell carcinoma. Cancer Res, 2004. 64(1): p. 35-9.

246. Yoo, Y.G., et al., Novel function of orphan nuclear receptor Nur77 in stabilizing hypoxia-inducible factor-1alpha. J Biol Chem, 2004. 279(51): p. 53365-73.

247. Wu, Q., et al., Inhibition of trans-retinoic acid-resistant human breast cancer cell growth by retinoid X receptor-selective retinoids. Mol Cell Biol, 1997. 17(11): $\mathrm{p}$. 6598-608.

248. Forman, B.M., et al., Unique response pathways are established by allosteric interactions among nuclear hormone receptors. Cell, 1995. 81(4): p. 541-50.

249. Zhao, W.X., et al., Orphan receptor TR 3 attenuates the p300-induced acetylation of retinoid X receptor-alpha. Mol Endocrinol, 2007. 21(12): p. 2877-89.

250. Adlakha, Y.K., et al., Pro-apoptotic miRNA-128-2 modulates ABCA1, ABCG1 and RXRalpha expression and cholesterol homeostasis. Cell Death Dis, 2013. 4: p. e780.

251. Guerit, D., et al., Sox9-regulated miRNA-574-3p inhibits chondrogenic differentiation of mesenchymal stem cells. PLoS One, 2013. 8(4): p. e62582.

252. Shehadeh, L.A., et al., MicroRNA-20a constrains p300-driven myocardial angiogenic transcription by direct targeting of p300. PLoS One, 2013. 8(11): $\mathrm{p}$. e79133.

253. Chiyomaru, T., et al., Genistein up-regulates tumor suppressor microRNA-574-3p in prostate cancer. PLoS One, 2013. 8(3): p. e58929.

254. Duan, Y., et al., miR-150 regulates high glucose-induced cardiomyocyte hypertrophy by targeting the transcriptional co-activator p300. Exp Cell Res, 2013. 319(3): p. 173-84.

255. Zhou, Y., et al., The miR-106b 25 cluster promotes bypass of doxorubicininduced senescence and increase in motility and invasion by targeting the Ecadherin transcriptional activator EP300. Cell Death Differ, 2014. 21(3): p. 46274.

256. Hu, Z., et al., miRNA-132-3p inhibits osteoblast differentiation by targeting Ep300 in simulated microgravity. Sci Rep, 2015. 5: p. 18655.

257. Ni, B., et al., Mycobacterium tuberculosis decreases human macrophage IFNgamma responsiveness through miR-132 and miR-26a. J Immunol, 2014. 193(9): p. 4537-47. 
258. Pan, Z., et al., MicroRNA-101 inhibited postinfarct cardiac fibrosis and improved left ventricular compliance via the FBJ osteosarcoma oncogene/transforming growth factor-betal pathway. Circulation, 2012. 126(7): p. 840-50.

259. Tao, T., et al., Involvement of FOS-mediated miR-181b/miR-21 signalling in the progression of malignant gliomas. Eur J Cancer, 2013. 49(14): p. 3055-63.

260. Liang, X., et al., miR-101 inhibits the G1-to-S phase transition of cervical cancer cells by targeting Fos. Int J Gynecol Cancer, 2014. 24(7): p. 1165-72.

261. Konno, Y., et al., MicroRNA-101 targets EZH2, MCL-1 and FOS to suppress proliferation, invasion and stem cell-like phenotype of aggressive endometrial cancer cells. Oncotarget, 2014. 5(15): p. 6049-62.

262. Liu, J.J., et al., A novel AP-1/miR-101 regulatory feedback loop and its implication in the migration and invasion of hepatoma cells. Nucleic Acids Res, 2014. 42(19): p. 12041-51.

263. Dunand-Sauthier, I., et al., Silencing of c-Fos expression by microRNA-155 is critical for dendritic cell maturation and function. Blood, 2011. 117(17): p. 4490500.

264. Taguchi, A., et al., Identification of hypoxia-inducible factor-1 alpha as a novel target for miR-17-92 microRNA cluster. Cancer Res, 2008. 68(14): p. 5540-5.

265. Krutilina, R., et al., MicroRNA-18a inhibits hypoxia-inducible factor lalpha activity and lung metastasis in basal breast cancers. Breast Cancer Res, 2014. 16(4): p. R78.

266. Han, F., Y. Wu, and W. Jiang, MicroRNA-18a Decreases Choroidal Endothelial Cell Proliferation and Migration by Inhibiting HIF1A Expression. Med Sci Monit, 2015. 21: p. 1642-7.

267. Lei, Z., et al., Regulation of HIF-lalpha and VEGF by miR-20b tunes tumor cells to adapt to the alteration of oxygen concentration. PLoS One, 2009. 4(10): $\mathrm{p}$. e7629.

268. Shen, J., et al., MicroRNAs regulate ocular neovascularization. Mol Ther, 2008. 16(7): p. 1208-16.

269. Song, T., et al., MiR-138 suppresses expression of hypoxia-inducible factor lalpha (HIF-1alpha) in clear cell renal cell carcinoma 786-O cells. Asian Pac J Cancer Prev, 2011. 12(5): p. 1307-11.

270. Yeh, Y.M., et al., MicroRNA-138 suppresses ovarian cancer cell invasion and metastasis by targeting SOX4 and HIF-1alpha. Int J Cancer, 2013. 133(4): p. 86778.

271. Bruning, U., et al., MicroRNA-155 promotes resolution of hypoxia-inducible factor lalpha activity during prolonged hypoxia. Mol Cell Biol, 2011. 31(19): $\mathrm{p}$. 4087-96.

272. Jia, X.Q., et al., Lentivirus-mediated overexpression of microRNA-199a inhibits cell proliferation of human hepatocellular carcinoma. Cell Biochem Biophys, 2012. 62(1): p. 237-44.

273. Wang, C., et al., Underexpressed microRNA-199b-5p targets hypoxia-inducible factor-1alpha in hepatocellular carcinoma and predicts prognosis of hepatocellular carcinoma patients. J Gastroenterol Hepatol, 2011. 26(11): p. 1630-7. 
274. Shang, W., et al., MiR199b suppresses expression of hypoxia-inducible factor lalpha (HIF-lalpha) in prostate cancer cells. Int J Mol Sci, 2013. 14(4): p. 842236.

275. Yue, J., et al., MicroRNA-206 is involved in hypoxia-induced pulmonary hypertension through targeting of the HIF-1alpha/Fhl-1 pathway. Lab Invest, 2013. 93(7): p. 748-59.

276. Wang, H., et al., Negative regulation of Hifla expression and TH17 differentiation by the hypoxia-regulated microRNA miR-210. Nat Immunol, 2014. 15(4): p. 393-401.

277. Liu, F.J., et al., MiR-335 Regulates Hif-1alpha to Reduce Cell Death in Both Mouse Cell Line and Rat Ischemic Models. PLoS One, 2015. 10(6): p. e0128432.

278. Xu, H., et al., MiR-338-3p inhibits hepatocarcinoma cells and sensitizes these cells to sorafenib by targeting hypoxia-induced factor lalpha. PLoS One, 2014. 9(12): p. e115565.

279. Cha, S.T., et al., MicroRNA-519c suppresses hypoxia-inducible factor-lalpha expression and tumor angiogenesis. Cancer Res, 2010. 70(7): p. 2675-85.

280. Kim, N.H., et al., p53 and microRNA-34 are suppressors of canonical Wnt signaling. Sci Signal, 2011. 4(197): p. ra71.

281. Yang, W. and A.J. Yee, Versican 3'-untranslated region (3'UTR) promotes dermal wound repair and fibroblast migration by regulating miRNA activity. Biochim Biophys Acta, 2014. 1843(7): p. 1373-85.

282. Saydam, O., et al., Downregulated microRNA-200a in meningiomas promotes tumor growth by reducing E-cadherin and activating the Wnt/beta-catenin signaling pathway. Mol Cell Biol, 2009. 29(21): p. 5923-40.

283. Su, J., et al., MicroRNA-200a suppresses the Wnt/beta-catenin signaling pathway by interacting with beta-catenin. Int J Oncol, 2012. 40(4): p. 1162-70.

284. Liu, J., et al., Downregulation of miR-200a induces EMT phenotypes and CSClike signatures through targeting the beta-catenin pathway in hepatic oval cells. PLoS One, 2013. 8(11): p. e79409.

285. Xia, H., L.L. Ooi, and K.M. Hui, MiR-214 targets beta-catenin pathway to suppress invasion, stem-like traits and recurrence of human hepatocellular carcinoma. PLoS One, 2012. 7(9): p. e44206.

286. Wang, X., et al., MiR-214 inhibits cell growth in hepatocellular carcinoma through suppression of beta-catenin. Biochem Biophys Res Commun, 2012. 428(4): p. 525-31.

287. Wu, M.Y., et al., MiR-34a regulates therapy resistance by targeting HDACl and HDAC7 in breast cancer. Cancer Lett, 2014. 354(2): p. 311-9.

288. Zhao, J., et al., TP53-independent function of miR-34a via HDACl and p21(CIP1/WAF1.). Mol Ther, 2013. 21(9): p. 1678-86.

289. Noonan, E.J., et al., miR-449a targets HDAC-1 and induces growth arrest in prostate cancer. Oncogene, 2009. 28(14): p. 1714-24.

290. Liu, T., et al., Epigenetic silencing of HDACl by miR-449a upregulates Runx2 and promotes osteoblast differentiation. Int J Mol Med, 2015. 35(1): p. 238-46.

291. Buggele, W.A., K.E. Krause, and C.M. Horvath, Small RNA profiling of influenza $A$ virus-infected cells identifies miR-449b as a regulator of histone deacetylase 1 and interferon beta. PLoS One, 2013. 8(9): p. e76560. 
292. Shen, Q., et al., Downregulation of histone deacetylase 1 by microRNA-520h contributes to the chemotherapeutic effect of doxorubicin. FEBS Lett, 2014. 588(1): p. 184-91.

293. Nohata, N., et al., Tumour-suppressive microRNA-874 contributes to cell proliferation through targeting of histone deacetylase 1 in head and neck squamous cell carcinoma. Br J Cancer, 2013. 108(8): p. 1648-58.

294. Kushwaha, P., et al., MicroRNA 874-3p Exerts Skeletal Anabolic Effects Epigenetically during Weaning by Suppressing Hdacl Expression. J Biol Chem, 2016. 291(8): p. 3959-66.

295. Jeong, B.C., et al., MicroRNA-194 reciprocally stimulates osteogenesis and inhibits adipogenesis via regulating COUP-TFII expression. Cell Death Dis, 2014. 5: p. e1532.

296. Kang, I.H., et al., MicroRNA-302a stimulates osteoblastic differentiation by repressing COUP-TFII expression. J Cell Physiol, 2015. 230(4): p. 911-21.

297. Tseng, S.H., et al., K14-EGFP-miR-31 transgenic mice have high susceptibility to chemical-induced squamous cell tumorigenesis that is associating with Ku80 repression. Int J Cancer, 2015. 136(6): p. 1263-75.

298. Zhang, Z.Y., et al., By downregulating Ku80, hsa-miR-526b suppresses non-small cell lung cancer. Oncotarget, 2015. 6(3): p. 1462-77.

299. Kumar, M., et al., Negative regulation of the tumor suppressor p53 gene by microRNAs. Oncogene, 2011. 30(7): p. 843-53.

300. Le, M.T., et al., MicroRNA-125b is a novel negative regulator of $p 53$. Genes Dev, 2009. 23(7): p. 862-76.

301. Zhang, N., X. Wei, and L. Xu, miR-150 promotes the proliferation of lung cancer cells by targeting P53. FEBS Lett, 2013. 587(15): p. 2346-51.

302. Wang, D.T., et al., miR-150, p53 protein and relevant miRNAs consist of a regulatory network in NSCLC tumorigenesis. Oncol Rep, 2013. 30(1): p. 492-8.

303. Liu, Y., et al., miR-375 targets the 53 gene to regulate cellular response to ionizing radiation and etoposide in gastric cancer cells. DNA Repair (Amst), 2013. 12(9): p. 741-50.

304. $\mathrm{Hu}, \mathrm{W}$., et al., Negative regulation of tumor suppressor p53 by microRNA miR504. Mol Cell, 2010. 38(5): p. 689-99.

305. Tian, S., et al., MicroRNA-1285 inhibits the expression of p53 by directly targeting its 3' untranslated region. Biochem Biophys Res Commun, 2010. 396(2): p. 435-9.

306. Lao, G., et al., Mir-155 promotes cervical cancer cell proliferation through suppression of its target gene LKB1. Tumour Biol, 2014. 35(12): p. 11933-8.

307. Zhang, X., et al., Regulation of the microRNA 200b (miRNA-200b) by transcriptional regulators $P E A 3$ and ELK-1 protein affects expression of Pin 1 protein to control anoikis. J Biol Chem, 2013. 288(45): p. 32742-52.

308. Luo, M.L., et al., Prolyl isomerase Pinl acts downstream of miR200c to promote cancer stem-like cell traits in breast cancer. Cancer Res, 2014. 74(13): p. 360316.

309. Lee, K.H., et al., MicroRNA-296-5p (miR-296-5p) functions as a tumor suppressor in prostate cancer by directly targeting Pin1. Biochim Biophys Acta, 2014. 1843(9): p. 2055-66. 
310. Liu, R., et al., Overexpressed microRNA-182 promotes proliferation and invasion in prostate cancer $P C-3$ cells by down-regulating $N$-myc downstream regulated gene 1 (NDRG1). PLoS One, 2013. 8(7): p. e68982.

311. Luo, E.C., et al., MicroRNA-769-3p down-regulates NDRG1 and enhances apoptosis in MCF-7 cells during reoxygenation. Sci Rep, 2014. 4: p. 5908.

312. Zhang, K.L., et al., Blockage of a miR-21/EGFR regulatory feedback loop augments anti-EGFR therapy in glioblastomas. Cancer Lett, 2014. 342(1): p. 13949.

313. Xiao, J., et al., miR-141 modulates androgen receptor transcriptional activity in human prostate cancer cells through targeting the small heterodimer partner protein. Prostate, 2012. 72(14): p. 1514-22.

314. Lin, T., et al., MicroRNA-378g enhanced radiosensitivity of NPC cells partially by targeting protein tyrosine phosphatase SHP-1. Int J Radiat Biol, 2015. 91(11): p. 859-66.

315. Pan, X., et al., MicroRNA-4649-3p inhibits cell proliferation by targeting protein tyrosine phosphatase SHP-1 in nasopharyngeal carcinoma cells. Int J Mol Med, 2015. 36(2): p. 559-64.

316. Martin, E.C., et al., Preferential star strand biogenesis of pre-miR-24-2 targets $P K C$-alpha and suppresses cell survival in MCF-7 breast cancer cells. Mol Carcinog, 2014. 53(1): p. 38-48.

317. Jin, Y., et al., MicroRNA-99 family targets AKT/mTOR signaling pathway in dermal wound healing. PLoS One, 2013. 8(5): p. e64434.

318. Shen, G., et al., MicroRNA-105 suppresses cell proliferation and inhibits PI3K/AKT signaling in human hepatocellular carcinoma. Carcinogenesis, 2014. 35(12): p. 2748-55.

319. Chen, X.N., et al., MiR-133b regulates bladder cancer cell proliferation and apoptosis by targeting Bcl-w and Akt1. Cancer Cell Int, 2014. 14: p. 70.

320. Noguchi, S., et al., Replacement treatment with microRNA-143 and-145 induces synergistic inhibition of the growth of human bladder cancer cells by regulating PI3K/Akt and MAPK signaling pathways. Cancer Lett, 2013. 328(2): p. 353-61.

321. Lin, R.J., Y.C. Lin, and A.L. Yu, miR-149* induces apoptosis by inhibiting Akt1 and E2F1 in human cancer cells. Mol Carcinog, 2010. 49(8): p. 719-27.

322. Wei, Y., et al., The microRNA-342-5p fosters inflammatory macrophage activation through an Akt1- and microRNA-155-dependent pathway during atherosclerosis. Circulation, 2013. 127(15): p. 1609-19.

323. Zhang, L., et al., miR-92a inhibits vascular smooth muscle cell apoptosis: role of the MKK4-JNK pathway. Apoptosis, 2014. 19(6): p. 975-83.

324. Yan, D., et al., Targeting DNA-PKcs and ATM with miR-101 sensitizes tumors to radiation. PLoS One, 2010. 5(7): p. e11397.

325. Zhang, J., C. Han, and T. Wu, MicroRNA-26a promotes cholangiocarcinoma growth by activating beta-catenin. Gastroenterology, 2012. 143(1): p. 246-56 e8.

326. Wang, Q., et al., miR-346 regulates osteogenic differentiation of human bone marrow-derived mesenchymal stem cells by targeting the Wnt/beta-catenin pathway. PLoS One, 2013. 8(9): p. e72266. 
327. Du, X., et al., microRNA-195 inhibits the proliferation, migration and invasion of cervical cancer cells via the inhibition of CCND2 and MYB expression. Oncol Lett, 2015. 10(4): p. 2639-2643.

328. Lee, N.S., et al., miR-302b maintains "stemness" of human embryonal carcinoma cells by post-transcriptional regulation of Cyclin D2 expression. Biochem Biophys Res Commun, 2008. 377(2): p. 434-40.

329. Kota, J., et al., Therapeutic microRNA delivery suppresses tumorigenesis in a murine liver cancer model. Cell, 2009. 137(6): p. 1005-17.

330. Li, X., et al., miR-340 inhibits glioblastoma cell proliferation by suppressing CDK6, cyclin-D1 and cyclin-D2. Biochem Biophys Res Commun, 2015. 460(3): p. 670-7.

331. Wang, J., et al., miR-198 Represses the Proliferation of HaCaT Cells by Targeting Cyclin D2. Int J Mol Sci, 2015. 16(8): p. 17018-28.

332. Bhinge, A., et al., MiR-375 is Essential for Human Spinal Motor Neuron Development and May Be Involved in Motor Neuron Degeneration. Stem Cells, 2016. 34(1): p. 124-34.

333. Mo, X., et al., MicroRNA-610 suppresses the proliferation of human glioblastoma cells by repressing CCND2 and AKT3. Mol Med Rep, 2016. 13(3): p. 1961-6.

334. Zhou, J., et al., miR-26a regulates mouse hepatocyte proliferation via directly targeting the 3' untranslated region of CCND2 and CCNE2. Hepatobiliary Pancreat Dis Int, 2016. 15(1): p. 65-72.

335. Wei, W., et al., The NF-kappaB-modulated microRNAs miR-195 and miR-497 inhibit myoblast proliferation by targeting Igflr, Insr and cyclin genes. J Cell Sci, 2016. 129(1): p. 39-50.

336. Lovat, F., et al., miR-15b/16-2 deletion promotes B-cell malignancies. Proc Natl Acad Sci U S A, 2015. 112(37): p. 11636-41.

337. Dong, Q., et al., MicroRNA let-7a inhibits proliferation of human prostate cancer cells in vitro and in vivo by targeting E2F2 and CCND2. PLoS One, 2010. 5(4): p. e10147.

338. Yan, D., et al., Role of microRNA-182 in posterior uveal melanoma: regulation of tumor development through MITF, BCL2 and cyclin D2. PLoS One, 2012. 7(7): p. e40967.

339. Gong, J., et al., Characterization of microRNA-29 family expression and investigation of their mechanistic roles in gastric cancer. Carcinogenesis, 2014. 35(2): p. 497-506.

340. Cao, X., et al., MicroRNA profiling during rat ventricular maturation: A role for miR-29a in regulating cardiomyocyte cell cycle re-entry. FEBS Lett, 2013. 587(10): p. 1548-55.

341. Chen, X., et al., MicroRNA-124a is epigenetically regulated and acts as a tumor suppressor by controlling multiple targets in uveal melanoma. Invest Ophthalmol Vis Sci, 2013. 54(3): p. 2248-56.

342. Huang, S., et al., Attenuation of microRNA-16 derepresses the cyclins D1, D2 and E1 to provoke cardiomyocyte hypertrophy. J Cell Mol Med, 2015. 19(3): p. 60819. 
343. Wu, X., et al., MiR-204, down-regulated in retinoblastoma, regulates proliferation and invasion of human retinoblastoma cells by targeting CyclinD2 and MMP-9. FEBS Lett, 2015. 589(5): p. 645-50.

344. Yan, G., et al., MicroRNA-145 suppresses mouse granulosa cell proliferation by targeting activin receptor IB. FEBS Lett, 2012. 586(19): p. 3263-70.

345. Yang, S.L., et al., MiR-302/367 regulate neural progenitor proliferation, differentiation timing, and survival in neurulation. Dev Biol, 2015. 408(1): p. $140-50$.

346. Poudel, S., et al., Sulfuretin-induced miR-30C selectively downregulates cyclin D1 and D2 and triggers cell death in human cancer cell lines. Biochem Biophys Res Commun, 2013. 431(3): p. 572-8.

347. Zhou, J., et al., miR-206 is down-regulated in breast cancer and inhibits cell proliferation through the up-regulation of cyclinD2. Biochem Biophys Res Commun, 2013. 433(2): p. 207-12.

348. Zhang, L., et al., miR-206 inhibits gastric cancer proliferation in part by repressing cyclinD2. Cancer Lett, 2013. 332(1): p. 94-101.

349. Yang, Y., et al., Thioredoxin 1 negatively regulates angiotensin II-induced cardiac hypertrophy through upregulation of miR-98/let-7. Circ Res, 2011. 108(3): p. 305-13.

350. Leone, V., et al., MiR-1 is a tumor suppressor in thyroid carcinogenesis targeting CCND2, CXCR4, and SDF-1alpha. J Clin Endocrinol Metab, 2011. 96(9): p. E1388-98.

351. Wang, L., et al., The role of TXNDC5 in castration-resistant prostate cancerinvolvement of androgen receptor signaling pathway. Oncogene, 2015. 34(36): $\mathrm{p}$. 4735-45.

352. Ma, Q., et al., microRNA-16 represses colorectal cancer cell growth in vitro by regulating the p53/survivin signaling pathway. Oncol Rep, 2013. 29(4): p. 16528.

353. Bian, K., et al., MicroRNA-203 leads to G1 phase cell cycle arrest in laryngeal carcinoma cells by directly targeting survivin. FEBS Lett, 2012. 586(6): p. 804-9.

354. Itesako, T., et al., The microRNA expression signature of bladder cancer by deep sequencing: the functional significance of the miR-195/497 cluster. PLoS One, 2014. 9(2): p. e84311.

355. Alajez, N.M., et al., MiR-218 suppresses nasopharyngeal cancer progression through downregulation of survivin and the SLIT2-ROBO1 pathway. Cancer Res, 2011. 71(6): p. 2381-91.

356. Kogo, R., et al., The microRNA-218 Survivin axis regulates migration, invasion, and lymph node metastasis in cervical cancer. Oncotarget, 2015. 6(2): p. 1090100.

357. $\mathrm{Hu}, \mathrm{Y} ., \mathrm{K} . \mathrm{Xu}$, and $\mathrm{E}$. Yague, miR-218 targets survivin and regulates resistance to chemotherapeutics in breast cancer. Breast Cancer Res Treat, 2015. 151(2): $\mathrm{p}$. 269-80.

358. Zhang, X., et al., MicroRNA-203 Is a Prognostic Indicator in Bladder Cancer and Enhances Chemosensitivity to Cisplatin via Apoptosis by Targeting Bcl-w and Survivin. PLoS One, 2015. 10(11): p. e0143441. 
359. Zhang, Y., et al., miR-203 inhibits proliferation and self-renewal of leukemia stem cells by targeting survivin and Bmi-1. Sci Rep, 2016. 6: p. 19995.

360. Althoff, K., et al., miR-542-3p exerts tumor suppressive functions in neuroblastoma by downregulating Survivin. Int J Cancer, 2015. 136(6): p. 130820.

361. Saini, S., et al., MicroRNA-708 induces apoptosis and suppresses tumorigenicity in renal cancer cells. Cancer Res, 2011. 71(19): p. 6208-19.

362. Pulikkan, J.A., et al., Cell-cycle regulator E2F1 and microRNA-223 comprise an autoregulatory negative feedback loop in acute myeloid leukemia. Blood, 2010. 115(9): p. 1768-78.

363. Dar, A.A., et al., miRNA-205 suppresses melanoma cell proliferation and induces senescence via regulation of E2F1 protein. J Biol Chem, 2011. 286(19): p. 1660614.

364. Piovan, C., et al., Oncosuppressive role of p53-induced miR-205 in triple negative breast cancer. Mol Oncol, 2012. 6(4): p. 458-72.

365. Lee, K.H., et al., MicroRNA-330 acts as tumor suppressor and induces apoptosis of prostate cancer cells through E2F1-mediated suppression of Akt phosphorylation. Oncogene, 2009. 28(38): p. 3360-70.

366. Guo, X., et al., miRNA-331-3p directly targets E2F1 and induces growth arrest in human gastric cancer. Biochem Biophys Res Commun, 2010. 398(1): p. 1-6.

367. Chen, W., et al., MiR-136 targets E2F1 to reverse cisplatin chemosensitivity in glioma cells. J Neurooncol, 2014. 120(1): p. 43-53.

368. Yin, M., et al., Transactivation of micrornA-320 by microRNA-383 regulates granulosa cell functions by targeting E2F1 and SF-1 proteins. J Biol Chem, 2014. 289(26): p. 18239-57.

369. Xiao, B., et al., MiRNA-329 targeting E2F1 inhibits cell proliferation in glioma cells. J Transl Med, 2013. 11: p. 172.

370. Gu, Y., et al., MicroRNA-493 suppresses tumor growth, invasion and metastasis of lung cancer by regulating E2F1. PLoS One, 2014. 9(8): p. e102602.

371. Yang, G., et al., MiR-106a inhibits glioma cell growth by targeting E2F1 independent of p53 status. J Mol Med (Berl), 2011. 89(10): p. 1037-50.

372. Christensen, L.L., et al., MiRNA-362-3p induces cell cycle arrest through targeting of E2F1, USF2 and PTPN1 and is associated with recurrence of colorectal cancer. Int J Cancer, 2013. 133(1): p. 67-78.

373. D'Angelo, D., et al., Altered microRNA expression profile in human pituitary $G H$ adenomas: down-regulation of miRNA targeting HMGA1, HMGA2, and E2F1. J Clin Endocrinol Metab, 2012. 97(7): p. E1128-38.

374. Tai, M.C., et al., miR-342-3p regulates MYC transcriptional activity via direct repression of E2F1 in human lung cancer. Carcinogenesis, 2015. 36(12): p. 146473.

375. He, L., et al., A microRNA component of the p53 tumour suppressor network. Nature, 2007. 447(7148): p. 1130-4.

376. Deng, X., et al., miR-124 radiosensitizes human glioma cells by targeting CDK4. J Neurooncol, 2013. 114(3): p. 263-74.

377. Feng, T., et al., MiR-124 inhibits cell proliferation in breast cancer through downregulation of CDK4. Tumour Biol, 2015. 36(8): p. 5987-97. 
378. Zhang, T., et al., MiR-124 retards bladder cancer growth by directly targeting CDK4. Acta Biochim Biophys Sin (Shanghai), 2014. 46(12): p. 1072-9.

379. Wu, J., et al., MicroRNA-188 suppresses G1/S transition by targeting multiple cyclin/CDK complexes. Cell Commun Signal, 2014. 12: p. 66.

380. Lin, Y., et al., Cyclin-dependent kinase 4 is a novel target in micoRNA-195mediated cell cycle arrest in bladder cancer cells. FEBS Lett, 2012. 586(4): p. 442-7.

381. Georgantas, R.W., 3rd, et al., MicroRNA-206 induces G1 arrest in melanoma by inhibition of CDK4 and Cyclin D. Pigment Cell Melanoma Res, 2014. 27(2): p. 275-86.

382. Shao, Y., et al., Direct repression of the oncogene CDK4 by the tumor suppressor miR-486-5p in non-small cell lung cancer. Oncotarget, 2016.

383. Liu, G., et al., MiR-506 suppresses proliferation and induces senescence by directly targeting the CDK4/6-FOXM1 axis in ovarian cancer. J Pathol, 2014. 233(3): p. 308-18.

384. Li, D., et al., MiR-613 induces cell cycle arrest by targeting CDK4 in non-small cell lung cancer. Cell Oncol (Dordr), 2016. 39(2): p. 139-47.

385. Hargraves, K.G., L. He, and G.L. Firestone, Phytochemical regulation of the tumor suppressive microRNA, miR-34a, by p53-dependent and independent responses in human breast cancer cells. Mol Carcinog, 2016. 55(5): p. 486-98.

386. Xiao, H., et al., miR-206 functions as a novel cell cycle regulator and tumor suppressor in clear-cell renal cell carcinoma. Cancer Lett, 2016. 374(1): p. 10716.

387. Xiao, H., et al., MiR-1 downregulation correlates with poor survival in clear cell renal cell carcinoma where it interferes with cell cycle regulation and metastasis. Oncotarget, 2015. 6(15): p. 13201-15.

388. Martin, N.T., et al., ATM-dependent MiR-335 targets CtIP and modulates the DNA damage response. PLoS Genet, 2013. 9(5): p. e1003505.

389. Huhn, D., et al., miR-19, a component of the oncogenic miR-17 approximately 92 cluster, targets the DNA-end resection factor CtIP. Oncogene, 2015. 34(30): p. 3977-84.

390. Finlay-Schultz, J., et al., Progesterone downregulation of miR-141 contributes to expansion of stem-like breast cancer cells through maintenance of progesterone receptor and Stat5a. Oncogene, 2015. 34(28): p. 3676-87.

391. Dentelli, P., et al., microRNA-222 controls neovascularization by regulating signal transducer and activator of transcription $5 A$ expression. Arterioscler Thromb Vasc Biol, 2010. 30(8): p. 1562-8.

392. Pinatel, E.M., et al., miR-223 is a coordinator of breast cancer progression as revealed by bioinformatics predictions. PLoS One, 2014. 9(1): p. e84859.

393. Xu, C., et al., MiRNA-1469 promotes lung cancer cells apoptosis through targeting STAT5a. Am J Cancer Res, 2015. 5(3): p. 1180-9.

394. Christoffersen, N.R., et al., p53-independent upregulation of miR-34a during oncogene-induced senescence represses MYC. Cell Death Differ, 2010. 17(2): p. 236-45. 
395. Lal, A., et al., miR-24 Inhibits cell proliferation by targeting E2F2, MYC, and other cell-cycle genes via binding to "seedless" 3'UTR microRNA recognition elements. Mol Cell, 2009. 35(5): p. 610-25.

396. Sachdeva, M., et al., p53 represses c-Myc through induction of the tumor suppressor miR-145. Proc Natl Acad Sci U S A, 2009. 106(9): p. 3207-12.

397. Kong, Y.W., et al., The mechanism of micro-RNA-mediated translation repression is determined by the promoter of the target gene. Proc Natl Acad Sci U S A, 2008. 105(26): p. 8866-71.

398. Lujambio, A., et al., A microRNA DNA methylation signature for human cancer metastasis. Proc Natl Acad Sci U S A, 2008. 105(36): p. 13556-61.

399. Liu, Z., et al., The tumor-suppressive microRNA-135b targets c-myc in osteoscarcoma. PLoS One, 2014. 9(7): p. e102621.

400. Lan, F.F., et al., Hsa-let-7g inhibits proliferation of hepatocellular carcinoma cells by downregulation of c-Myc and upregulation of p16(INK4A). Int J Cancer, 2011. 128(2): p. 319-31.

401. Liao, J.M. and H. Lu, Autoregulatory suppression of c-Myc by miR-185-3p. J Biol Chem, 2011. 286(39): p. 33901-9.

402. Lin, F., et al., Decrease expression of microRNA-744 promotes cell proliferation by targeting c-Myc in human hepatocellular carcinoma. Cancer Cell Int, 2014. 14: p. 58.

403. Wang, $\mathrm{H}$., et al., miR-320b suppresses cell proliferation by targeting c-Myc in human colorectal cancer cells. BMC Cancer, 2015. 15: p. 748.

404. He, W., et al., miR-494 acts as an anti-oncogene in gastric carcinoma by targeting c-myc. J Gastroenterol Hepatol, 2014. 29(7): p. 1427-34.

405. Liu, Y., et al., Ectopic expression of miR-494 inhibited the proliferation, invasion and chemoresistance of pancreatic cancer by regulating SIRT1 and c-Myc. Gene Ther, 2015. 22(9): p. 729-38.

406. Yuan, J., K. Wang, and M. Xi, MiR-494 Inhibits Epithelial Ovarian Cancer Growth by Targeting c-Myc. Med Sci Monit, 2016. 22: p. 617-24.

407. $\mathrm{Xi}, \mathrm{S}$., et al., Cigarette smoke mediates epigenetic repression of miR-487b during pulmonary carcinogenesis. J Clin Invest, 2013. 123(3): p. 1241-61.

408. Min, D.J., et al., MMSET stimulates myeloma cell growth through microRNAmediated modulation of c-MYC. Leukemia, 2013. 27(3): p. 686-94.

409. Chen, L., et al., Chromodomain helicaseladenosine triphosphatase DNA binding protein 1-like (CHD1l) gene suppresses the nucleus-to-mitochondria translocation of nur77 to sustain hepatocellular carcinoma cell survival. Hepatology, 2009. 50(1): p. 122-9.

410. Gottschalk, A.J., et al., Poly(ADP-ribosyl)ation directs recruitment and activation of an ATP-dependent chromatin remodeler. Proc Natl Acad Sci U S A, 2009. 106(33): p. 13770-4.

411. Ahel, D., et al., Poly(ADP-ribose)-dependent regulation of DNA repair by the chromatin remodeling enzyme ALC1. Science, 2009. 325(5945): p. 1240-3.

412. Zhao, B.X., et al., p53 mediates the negative regulation of MDM2 by orphan receptor TR3. EMBO J, 2006. 25(24): p. 5703-15.

413. Budanov, A.V. and M. Karin, $p 53$ target genes sestrin1 and sestrin 2 connect genotoxic stress and mTOR signaling. Cell, 2008. 134(3): p. 451-60. 
414. Zhan, Y.Y., et al., The orphan nuclear receptor Nur77 regulates LKB1 localization and activates AMPK. Nat Chem Biol, 2012. 8(11): p. 897-904.

415. Chen, H.Z., et al., Prolyl isomerase Pin1 stabilizes and activates orphan nuclear receptor TR3 to promote mitogenesis. Oncogene, 2012. 31(23): p. 2876-87.

416. Li, G.D., et al., Negative regulation of transcription coactivator p300 by orphan receptor TR3. Nucleic Acids Res, 2007. 35(21): p. 7348-59.

417. Sun, Z., et al., Inhibition of beta-catenin signaling by nongenomic action of orphan nuclear receptor Nur77. Oncogene, 2012. 31(21): p. 2653-67.

418. Chen, H.Z., et al., The orphan receptor TR 3 suppresses intestinal tumorigenesis in mice by downregulating Wnt signalling. Gut, 2012. 61(5): p. 714-24.

419. Cha, Y.H., et al., MiRNA-34 intrinsically links p53 tumor suppressor and Wnt signaling. Cell Cycle, 2012. 11(7): p. 1273-81.

420. Lu, W.J., et al., NDRG1 promotes growth of hepatocellular carcinoma cells by directly interacting with GSK-3beta and Nur77 to prevent beta-catenin degradation. Oncotarget, 2015. 6(30): p. 29847-59.

421. Yeo, M.G., et al., Negative cross-talk between Nur77 and small heterodimer partner and its role in apoptotic cell death of hepatoma cells. Mol Endocrinol, 2005. 19(4): p. 950-63.

422. Kim, H., et al., A novel function of Nur77: physical and functional association with protein kinase C. Biochem Biophys Res Commun, 2006. 348(3): p. 950-6.

423. Wu, W.S., et al., Promyelocytic leukemia protein PML inhibits Nur77-mediated transcription through specific functional interactions. Oncogene, 2002. 21(24): p. 3925-33.

424. Melnick, A., et al., Identification of novel chromosomal rearrangements in acute myelogenous leukemia involving loci on chromosome 2p23, 15q22 and 17q21. Leukemia, 1999. 13(10): p. 1534-8.

425. Mu, Z.M., et al., PML, a growth suppressor disrupted in acute promyelocytic leukemia. Mol Cell Biol, 1994. 14(10): p. 6858-67.

426. Liu, J.H., Z.M. Mu, and K.S. Chang, PML suppresses oncogenic transformation of NIH/3T3 cells by activated neu. J Exp Med, 1995. 181(6): p. 1965-73.

427. Le, X.F., et al., Recombinant PML adenovirus suppresses growth and tumorigenicity of human breast cancer cells by inducing G1 cell cycle arrest and apoptosis. Oncogene, 1998. 16(14): p. 1839-49.

428. Wang, Z.G., et al., Role of PML in cell growth and the retinoic acid pathway. Science, 1998. 279(5356): p. 1547-51.

429. Guo, A., et al., The function of PML in p53-dependent apoptosis. Nat Cell Biol, 2000. 2(10): p. 730-6.

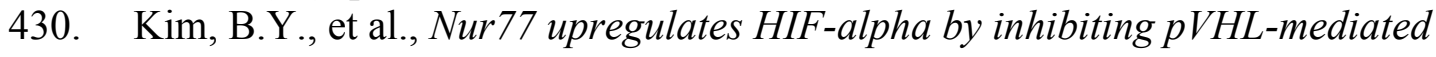
degradation. Exp Mol Med, 2008. 40(1): p. 71-83.

431. Chen, H.Z., et al., Akt phosphorylates the TR3 orphan receptor and blocks its targeting to the mitochondria. Carcinogenesis, 2008. 29(11): p. 2078-88.

432. Pekarsky, Y., et al., Akt phosphorylates and regulates the orphan nuclear receptor Nur77. Proc Natl Acad Sci U S A, 2001. 98(7): p. 3690-4.

433. Cheng, W., Y. Su, and F. Xu, CHDIL: a novel oncogene. Mol Cancer, 2013. 12(1): p. 170. 
434. Han, Y.H., et al., Regulation of Nur77 nuclear export by c-Jun N-terminal kinase and Akt. Oncogene, 2006. 25(21): p. 2974-86.

435. Zhou, Y., et al., Induction of Nur77-dependent apoptotic pathway by a coumarin derivative through activation of JNK and p38 MAPK. Carcinogenesis, 2014. 35(12): p. 2660-9.

436. Chang, L.F., et al., Overexpression of the orphan receptor Nur77 and its translocation induced by PCH4 may inhibit malignant glioma cell growth and induce cell apoptosis. J Surg Oncol, 2011. 103(5): p. 442-50.

437. Liu, P.Y., et al., Expression of Nur77 induced by an n-butylidenephthalide derivative promotes apoptosis and inhibits cell growth in oral squamous cell carcinoma. Invest New Drugs, 2012. 30(1): p. 79-89.

438. $\mathrm{Mu}, \mathrm{X}$. and $\mathrm{C}$. Chang, TR3 orphan nuclear receptor mediates apoptosis through up-regulating E2F1 in human prostate cancer LNCaP cells. J Biol Chem, 2003. 278(44): p. 42840-5.

439. Lee, S.O., et al., The orphan nuclear receptor NR4A1 (Nur77) regulates oxidative and endoplasmic reticulum stress in pancreatic cancer cells. Mol Cancer Res, 2014. 12(4): p. 527-38.

440. Zhang, L., et al., The influence of TXNDC5 gene on gastric cancer cell. J Cancer Res Clin Oncol, 2010. 136(10): p. 1497-505.

441. Saito-Hakoda, A., et al., Effects of RXR Agonists on Cell Proliferation/Apoptosis and ACTH Secretion/Pomc Expression. PLoS One, 2015. 10(12): p. e0141960.

442. Smith, A.G., et al., Regulation of NR4A nuclear receptor expression by oncogenic $B R A F$ in melanoma cells. Pigment Cell Melanoma Res, 2011. 24(3): p. 551-63.

443. Boudreaux, S.P., et al., Genome-wide profiling reveals transcriptional repression of MYC as a core component of NR4A tumor suppression in acute myeloid leukemia. Oncogenesis, 2012. 1: p. e19.

444. Kurakula, K., et al., NR4A nuclear receptors are orphans but not lonesome. Biochim Biophys Acta, 2014. 1843(11): p. 2543-2555.

445. Impey, S., et al., Defining the CREB regulon: a genome-wide analysis of transcription factor regulatory regions. Cell, 2004. 119(7): p. 1041-54.

446. Mazuy, C., et al., Palmitate increases Nur77 expression by modulating ZBP89 and Spl binding to the Nur77 proximal promoter in pancreatic beta-cells. FEBS Lett, 2013. 587(23): p. 3883-90.

447. Yan, G., et al., Orphan Nuclear Receptor Nur77 Inhibits Cardiac Hypertrophic Response to Beta-Adrenergic Stimulation. Mol Cell Biol, 2015. 35(19): p. 331223.

448. Qin, Q., et al., Orphan nuclear receptor Nur77 is a novel negative regulator of endothelin-1 expression in vascular endothelial cells. J Mol Cell Cardiol, 2014. 77: p. 20-8.

449. Liu, Y., et al., Nur77 suppresses pulmonary artery smooth muscle cell proliferation through inhibition of the STAT3/Pim-1/NFAT pathway. Am J Respir Cell Mol Biol, 2014. 50(2): p. 379-88.

450. Jacobs, C.M., et al., ERK2 prohibits apoptosis-induced subcellular translocation of orphan nuclear receptor NGFI-B/TR3. J Biol Chem, 2004. 279(48): p. 50097101. 
451. Fujii, Y., et al., ERK5 is involved in TCR-induced apoptosis through the modification of Nur77. Genes Cells, 2008. 13(5): p. 411-9.

452. Wang, A., et al., Phosphorylation of Nur77 by the MEK-ERK-RSK cascade induces mitochondrial translocation and apoptosis in T cells. J Immunol, 2009. 183(5): p. 3268-77.

453. Li, L., et al., Impeding the interaction between Nur77 and p38 reduces LPSinduced inflammation. Nat Chem Biol, 2015. 11(5): p. 339-46.

454. Niu, G., et al., Orphan nuclear receptor TR3/Nur77 improves wound healing by upregulating the expression of integrin beta4. FASEB J, 2015. 29(1): p. 131-40.

455. St. Jude Children's Research Hospital - Washington University. Pediatric Cancer Genome Project. 20 Aug 2014.

456. Zeng, F.Y., et al., PAX3-FKHR sensitizes human alveolar rhabdomyosarcoma cells to camptothecin-mediated growth inhibition and apoptosis. Cancer Lett, 2009. 284(2): p. 157-64.

457. Kotchetkov, R., et al., Increased malignant behavior in neuroblastoma cells with acquired multi-drug resistance does not depend on P-gp expression. Int J Oncol, 2005. 27(4): p. 1029-37.

458. Zhu, C.H., et al., Cellular senescence in human myoblasts is overcome by human telomerase reverse transcriptase and cyclin-dependent kinase 4: consequences in aging muscle and therapeutic strategies for muscular dystrophies. Aging Cell, 2007. 6(4): p. 515-23.

459. Lu, Z., et al., miR-301a as an NF-kappaB activator in pancreatic cancer cells. EMBO J, 2011. 30(1): p. 57-67.

460. Yu, J., et al., Induced pluripotent stem cell lines derived from human somatic cells. Science, 2007. 318(5858): p. 1917-20.

461. Abramoff, M.D., Magalhaes, P.J., Ram, S.J., Image Processing with ImageJ. Biophotonics International, 2004. volume 11( issue 7): p. 36-42

462. Hatten, M.E., Neuronal regulation of astroglial morphology and proliferation in vitro. J Cell Biol, 1985. 100(2): p. 384-96.

463. Murray, M.J., et al., Solid tumors of childhood display specific serum microRNA profiles. Cancer Epidemiol Biomarkers Prev, 2015. 24(2): p. 350-60.

464. Lagos-Quintana, M., et al., Identification of tissue-specific microRNAs from mouse. Curr Biol, 2002. 12(9): p. 735-9.

465. Pierson, J., et al., Regulation of cyclin dependent kinase 6 by microRNA 124 in medulloblastoma. J Neurooncol, 2008. 90(1): p. 1-7.

466. Li, K.K., et al., miR-124 is frequently down-regulated in medulloblastoma and is a negative regulator of SLC16A1. Hum Pathol, 2009. 40(9): p. 1234-43.

467. Silber, J., et al., Expression of miR-124 inhibits growth of medulloblastoma cells. Neuro Oncol, 2013. 15(1): p. 83-90.

468. Ke, N., et al., Nuclear hormone receptor NR4A2 is involved in cell transformation and apoptosis. Cancer Res, 2004. 64(22): p. 8208-12.

469. Bras, A., et al., Ceramide-induced cell death is independent of the Fas/Fas ligand pathway and is prevented by Nur77 overexpression in A20 B cells. Cell Death Differ, 2000. 7(3): p. 262-71. 
470. Kolluri, S.K., et al., Mitogenic Effect of Orphan Receptor TR3 and Its Regulation by MEKK1 in Lung Cancer Cells. Molecular and Cellular Biology, 2003. 23(23): p. 8651-8667.

471. Endo, T., Molecular mechanisms of skeletal muscle development, regeneration, and osteogenic conversion. Bone, 2015. 80: p. 2-13.

472. Braun, T. and M. Gautel, Transcriptional mechanisms regulating skeletal muscle differentiation, growth and homeostasis. Nat Rev Mol Cell Biol, 2011. 12(6): p. 349-61.

473. Bentzinger, C.F., Y.X. Wang, and M.A. Rudnicki, Building muscle: molecular regulation of myogenesis. Cold Spring Harb Perspect Biol, 2012. 4(2).

474. Chao, L.C., et al., Skeletal muscle Nur77 expression enhances oxidative metabolism and substrate utilization. J Lipid Res, 2012. 53(12): p. 2610-9.

475. Tontonoz, P., et al., The orphan nuclear receptor Nur77 is a determinant of myofiber size and muscle mass in mice. Mol Cell Biol, 2015. 35(7): p. 1125-38.

476. Yang, W.L. and R.W. Lim, Modulation of muscle creatine kinase promoter activity by the inducible orphan nuclear receptor TIS1. Biochem J, 1997. 321 ( Pt 2): p. 281-7.

477. Millay, D.P., et al., Myomaker is a membrane activator of myoblast fusion and muscle formation. Nature, 2013. 499(7458): p. 301-5.

478. Nuss, J.E., et al., Oxidative modification and aggregation of creatine kinase from aged mouse skeletal muscle. Aging (Albany NY), 2009. 1(6): p. 557-72.

479. Momken, I., et al., Impaired voluntary running capacity of creatine kinasedeficient mice. J Physiol, 2005. 565(Pt 3): p. 951-64.

480. Mazuy, C., et al., Palmitate increases Nur77 expression by modulating ZBP89 and Spl binding to the Nur77 proximal promoter in pancreatic beta-cells. FEBS Lett, 2013.

481. Mondanizadeh, M., et al., MicroRNA-124 Regulates Neuronal Differentiation of Mesenchymal Stem Cells by Targeting Sp1 mRNA. J Cell Biochem, 2015. 116(6): p. 943-53.

482. Packer, R.J. and G. Vezina, Management of and prognosis with medulloblastoma: therapy at a crossroads. Arch Neurol, 2008. 65(11): p. 1419-24.

483. Smoll, N.R., Relative survival of childhood and adult medulloblastomas and primitive neuroectodermal tumors (PNETS). Cancer, 2012. 118(5): p. 1313-22.

484. Taylor, M.D., et al., Molecular subgroups of medulloblastoma: the current consensus. Acta Neuropathol, 2012. 123(4): p. 465-72.

485. Ferretti, E., et al., Concerted microRNA control of Hedgehog signalling in cerebellar neuronal progenitor and tumour cells. EMBO J, 2008. 27(19): p. 2616-27.

486. Uziel, T., et al., The miR-17 92 cluster collaborates with the Sonic Hedgehog pathway in medulloblastoma. Proc Natl Acad Sci U S A, 2009. 106(8): p. 2812-7.

487. Gokhale, A., et al., Distinctive microRNA signature of medulloblastomas associated with the WNT signaling pathway. J Cancer Res Ther, 2010. 6(4): p. 521-9.

488. Ferretti, E., et al., MicroRNA profiling in human medulloblastoma. Int J Cancer, 2009. 124(3): p. 568-77. 
489. Makeyev, E.V., et al., The MicroRNA miR-124 promotes neuronal differentiation by triggering brain-specific alternative pre-mRNA splicing. Mol Cell, 2007. 27(3): p. 435-48.

490. $\mathrm{Xu}, \mathrm{W}$., et al., miR-124 regulates neural stem cells in the treatment of spinal cord injury. Neurosci Lett, 2012. 529(1): p. 12-7.

491. Silber, J., et al., miR-124 and miR-137 inhibit proliferation of glioblastoma multiforme cells and induce differentiation of brain tumor stem cells. BMC Med, 2008. 6: p. 14.

492. Wang, P., et al., Methylation-mediated silencing of the miR-124 genes facilitates pancreatic cancer progression and metastasis by targeting Racl. Oncogene, 2014. 33(4): p. 514-24.

493. Anwar, S.L., et al., Concordant hypermethylation of intergenic microRNA genes in human hepatocellular carcinoma as new diagnostic and prognostic marker. Int J Cancer, 2013. 133(3): p. 660-70.

494. Furuta, M., et al., miR-124 and miR-203 are epigenetically silenced tumorsuppressive microRNAs in hepatocellular carcinoma. Carcinogenesis, 2010. 31(5): p. 766-76.

495. Koukos, G., et al., MicroRNA-124 regulates STAT3 expression and is downregulated in colon tissues of pediatric patients with ulcerative colitis. Gastroenterology, 2013. 145(4): p. 842-52 e2.

496. Agirre, X., et al., Epigenetic silencing of the tumor suppressor microRNA HsamiR-124a regulates $C D K 6$ expression and confers a poor prognosis in acute lymphoblastic leukemia. Cancer Res, 2009. 69(10): p. 4443-53.

497. Maruoka, H., et al., Dibutyryl-cAMP up-regulates nur77 expression via histone modification during neurite outgrowth in PC12 cells. J Biochem, 2010. 148(1): p. 93-101.

498. Tomioka, T., et al., The histone deacetylase inhibitor trichostatin A induces neurite outgrowth in PC12 cells via the epigenetically regulated expression of the nur77 gene. Neurosci Res, 2014. 88: p. 39-48.

499. Hu, L.H., et al., Orphan nuclear receptor Nur77 Inhibits Oxidized LDL-induced differentiation of RAW264.7 murine macrophage cell line into dendritic like cells. BMC Immunol, 2014. 15(1): p. 54.

500. Jacobsen, P.F., D.J. Jenkyn, and J.M. Papadimitriou, Establishment of a human medulloblastoma cell line and its heterotransplantation into nude mice. $\mathrm{J}$ Neuropathol Exp Neurol, 1985. 44(5): p. 472-85.

501. Sato, A., et al., Cerebellar development transcriptome database (CDT-DB): profiling of spatio-temporal gene expression during the postnatal development of mouse cerebellum. Neural Netw, 2008. 21(8): p. 1056-69.

502. Human genomics. The Genotype-Tissue Expression (GTEx) pilot analysis: multitissue gene regulation in humans. Science, 2015. 348(6235): p. 648-60.

503. Pearen, M.A., et al., The orphan nuclear receptor, NOR-1, is a target of betaadrenergic signaling in skeletal muscle. Endocrinology, 2006. 147(11): p. 521727.

504. Ferran, B., et al., The nuclear receptor NOR-1 regulates the small muscle protein, X-linked (SMPX) and myotube differentiation. Sci Rep, 2016. 6: p. 25944. 
505. Duren, R.P., S.P. Boudreaux, and O.M. Conneely, Genome Wide Mapping of NR4A Binding Reveals Cooperativity with ETS Factors to Promote Epigenetic Activation of Distal Enhancers in Acute Myeloid Leukemia Cells. PLoS One, 2016. 11(3): p. e0150450.

506. Feo, S., et al., Transcription of the Human b Enolase Gene (ENO-3) Is Regulated by an Intronic Muscle-Specific Enhancer That Binds Myocyte-Specific Enhancer Factor 2 Proteins and Ubiquitous G-Rich-Box Binding Factors. Molecular and Cellular Biology, 1995. 15(11): p. 5991-6002. 


\section{APPENDIX. SUPPLEMENTAL DATA FOR CHAPTER 3}

Figure A-1. miR-124 decreases $N R 4 A 1$ expression in miRNA array. The Cancer miRNAs Transcriptome PCR Array containing cDNA from HeLa cells transfected with one of the 90 cancer-related miRNAs, as described in Materials and Methods, was used to detect $N R 4 A 1$ expression and identify miRNAs that target $N R 4 A 1$. The resulting gene expression of $N R 4 A 1$ is displayed as Log2 with horizontal lines indicating the cutoff value (as suggested by the manufacturer) at which $N R 4 A 1$ gene expression is considered to be significant. Three miRNAs, including miR-124, were found to decrease NR4AI expression. 


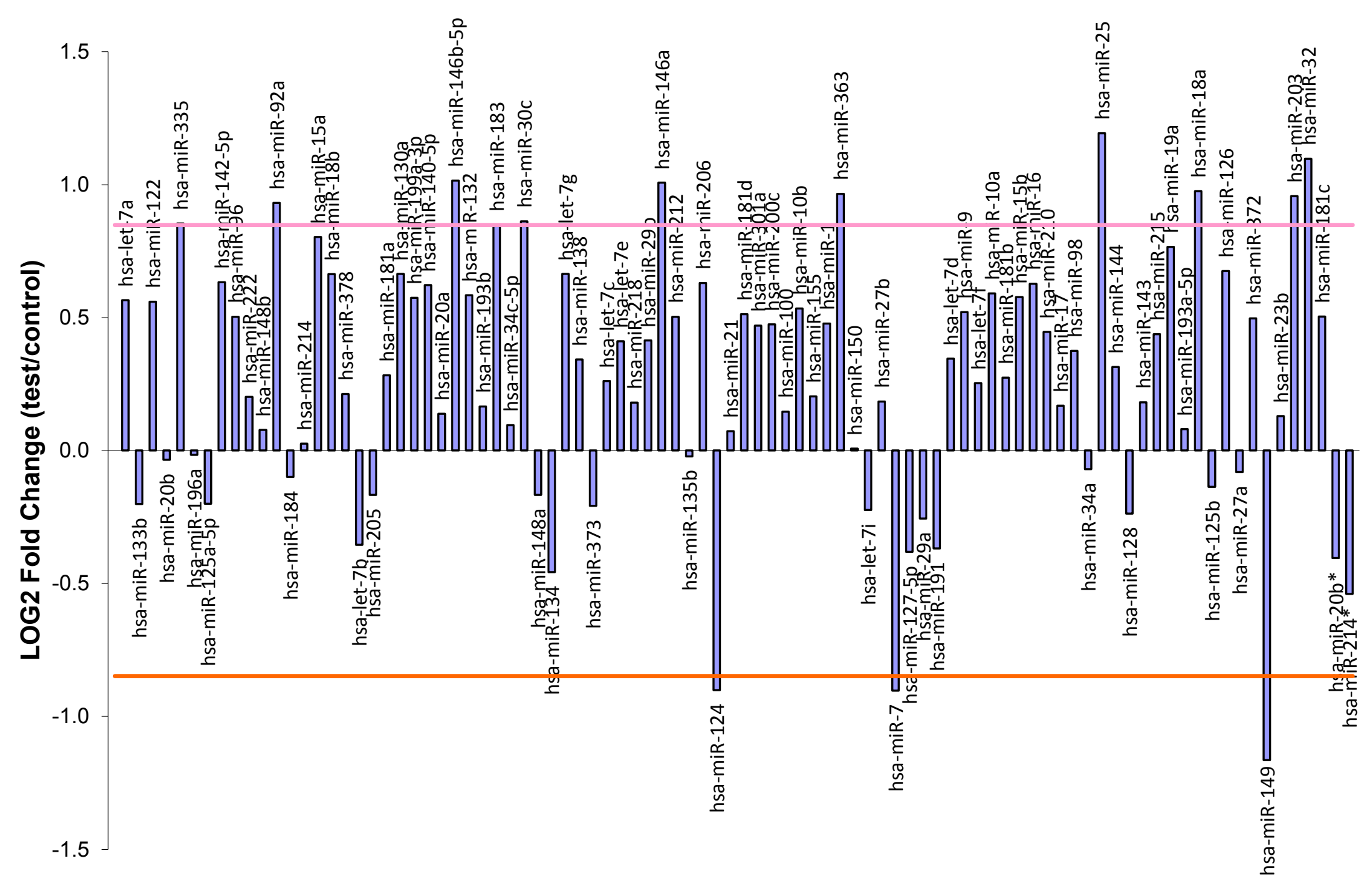



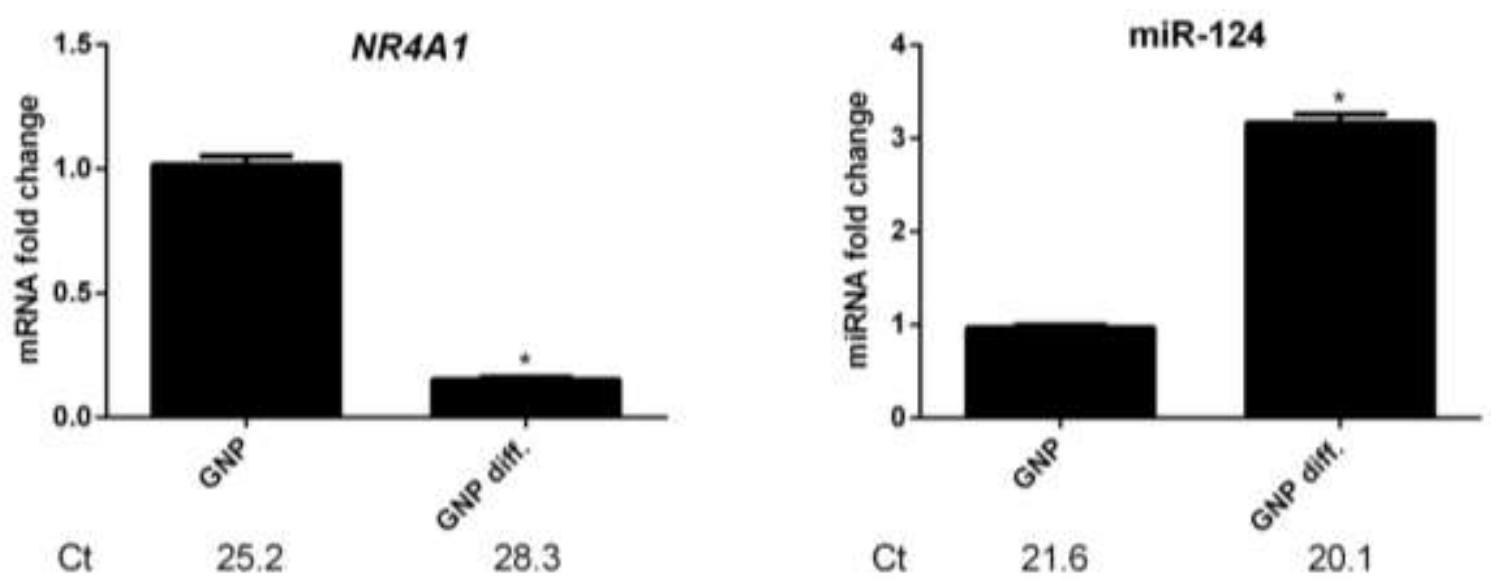

Figure A-2. NR4A1 and miR-124 have inverse expression in granule neurons. NR4A1 and miR-124 expression were measured in granule neuron precursors (GNPs) harvested from P7 mice. The GNPs were cultured for 24 hours, allowing enough time for the cells to differentiate (GNP diff.) before being collected for expression analysis. The fold change for the GNPs was set to 1 . The internal control for NR4A1 was GAPDH and the control for miR-124 was snoRNA 202. The data shown are the average of 3 independent experiments with the average $\mathrm{Ct}$ values indicated below each graph. * indicates $p<0.0001$. 


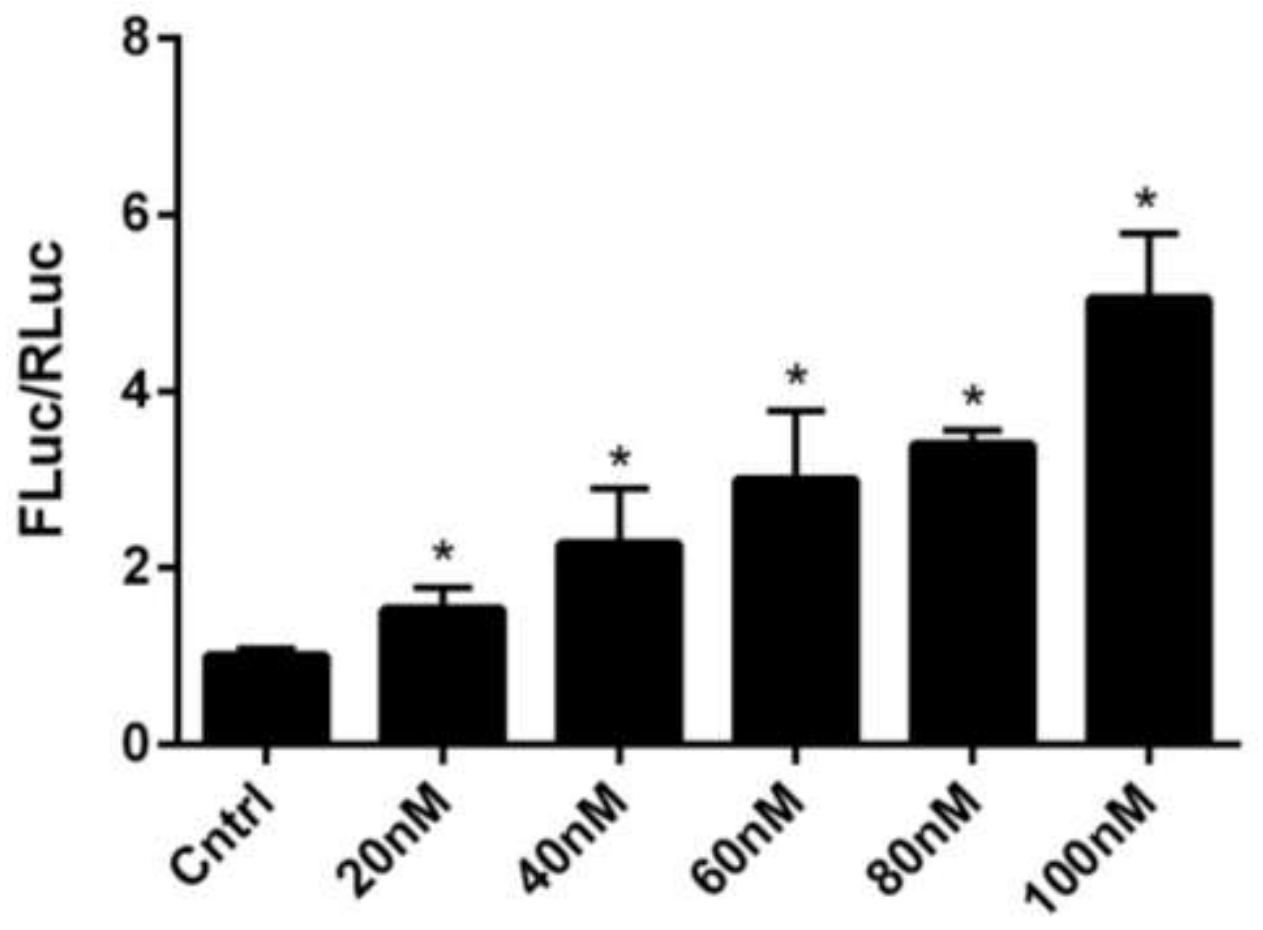

Figure A-3. An inhibitor of miR-124 increases NR4A1 activity.

Daoy cells were transfected with the NR4A1-3'UTR reporter plasmid (NR4A1-3'UTRLuc) and either the Exiqon miR-124 inhibitor at the indicated concentrations or the control molecule (Cntrl), resulting in increased luciferase activity as the concentration of the inhibitor increased. Data shown are representative of 2 independent experiments. * indicates $p<0.05$. 


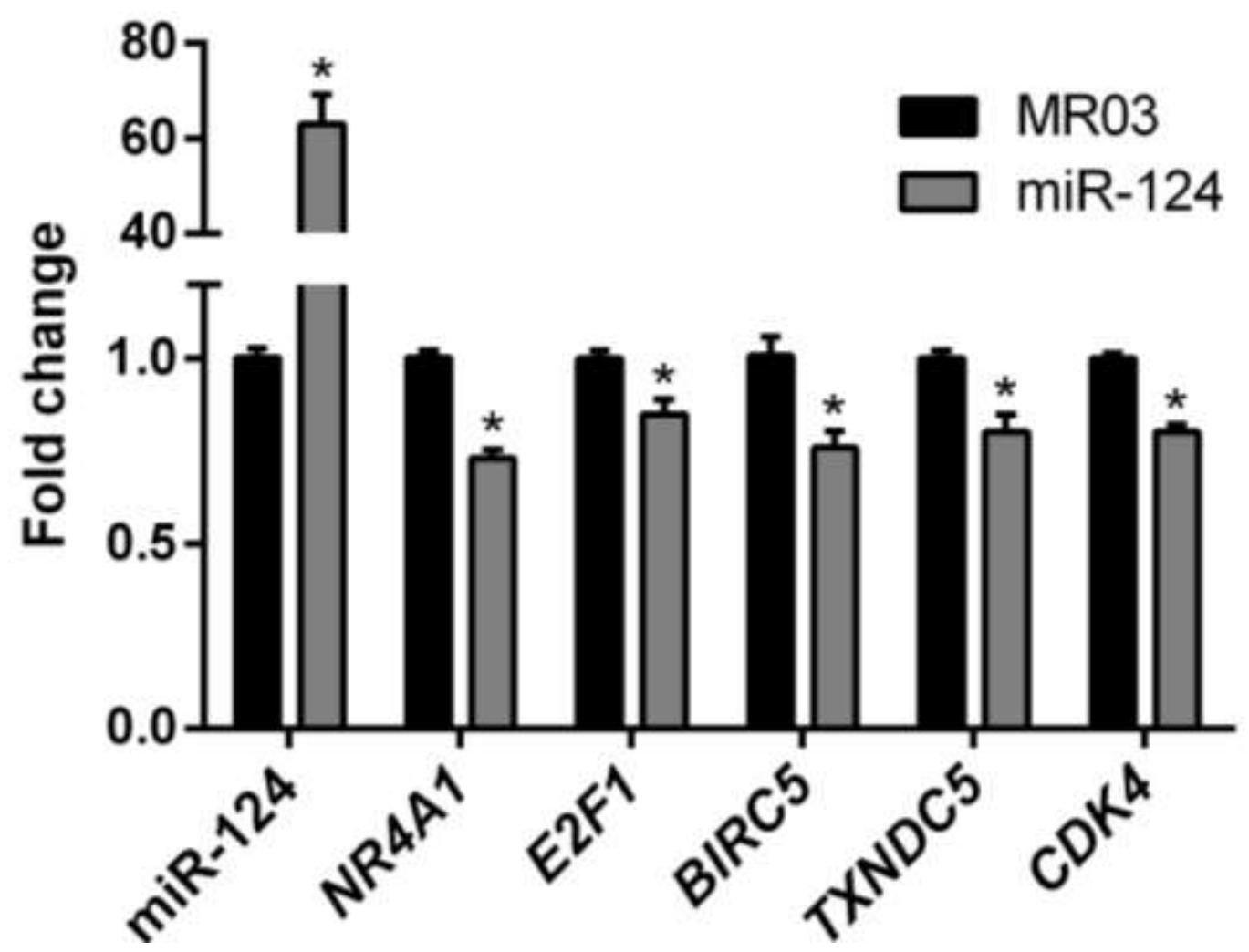

Figure A-4. miR-124 decreases levels of NR4A1 target genes in 293T cells.

Transfection of 293T cells with miR-124 decreased the levels of NR4AI and its target genes, E2F1, BIRC5 (survivin), TXNDC5, and CDK4, compared to those of cells transfected with the vector control (MR03). The data shown are the average of 3 independent experiments. * indicates $p<0.01$. 


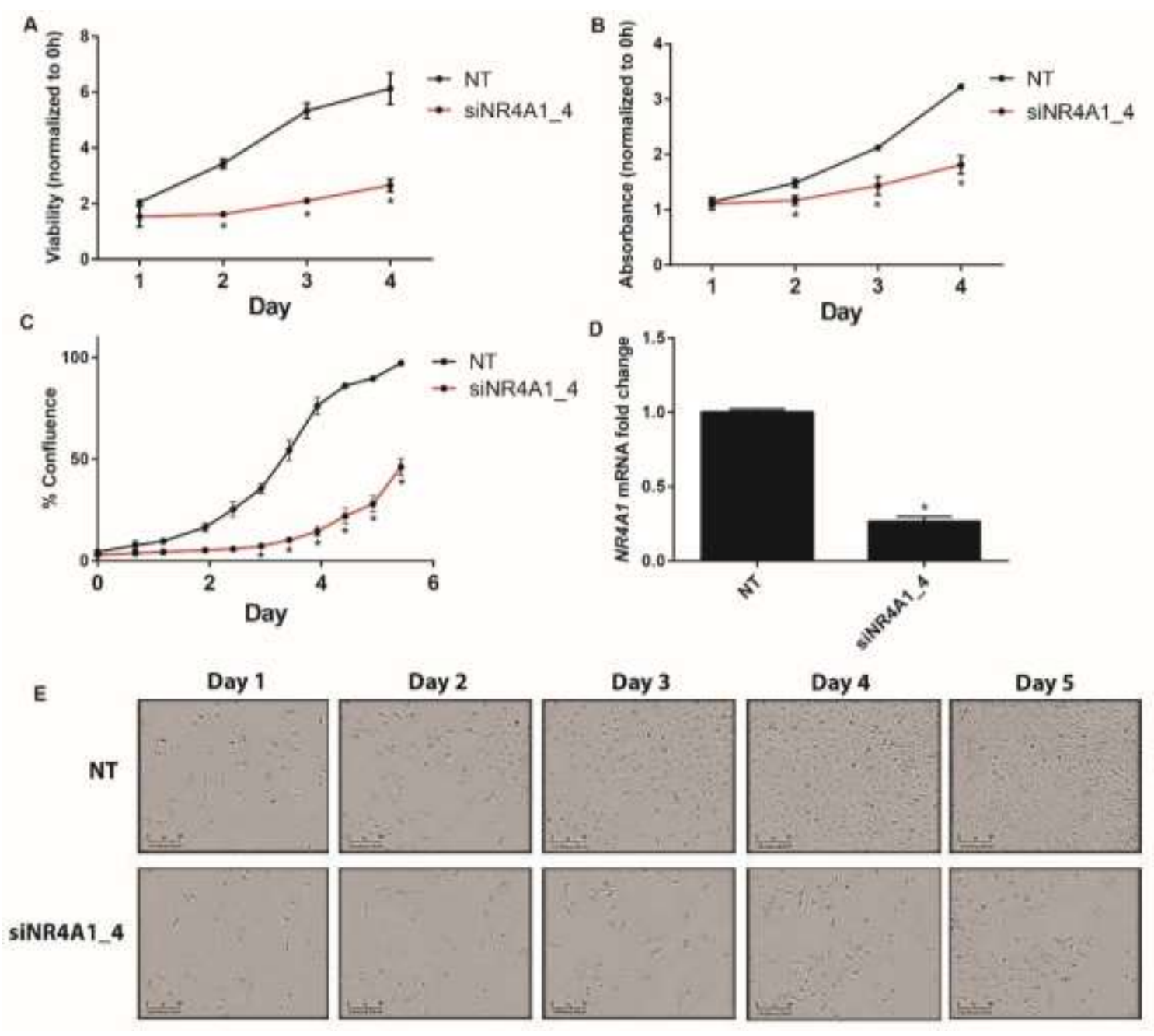

Figure A-5. NR4A1 knockdown decreases cell viability and proliferation.

(A) Daoy cells were transfected with $20 \mathrm{nM}$ of the individual siNR4A1_4 or nontargeting control (NT), and cell viability was measured via the CellTiter-Glo assay every day for 4 days. Viability for each day was normalized to that of Day 0 (0 hours), and statistical significance was calculated for each day; ${ }^{*} p<0.0001$. (B) Cells were stained with crystal violet every day for 4 days to measure proliferation over time. The absorbance was measured and normalized to that of Day 0 (0 hours). The statistical significance was calculated for each day; ${ }^{*} p<0.01$. (C) Proliferation was monitored via the IncuCyte live-cell imager. Cell confluence was averaged, with 4 replicates of each condition; * $p<0.0001$. (D) NR4A1 mRNA was significantly $(p<0.0001)$ decreased after transfecting Daoy cells with siNR4A1_4. (E) Images shown for each NT and siNR4A1_4 panel over 5 days are the same image view within the same well and are representative of 3 independent experiments with 4 wells for each condition. These images correspond to the data in C. Data shown in D are the average of 4 independent experiments. Data shown in $\mathrm{A}$ and $\mathrm{B}$ are representative of 3 independent experiments, and data in $\mathrm{C}$ and $\mathrm{E}$ are representative of 2 independent experiments. 


\section{VITA}

Alexa Farmer was born in Norfolk, VA in 1988 and grew up in Fairfax, VA. She graduated from Virginia Tech in 2011 with a Bachelor of Science in Biological Systems Engineering. She then started the Integrated Biomedical Sciences Program at the University of Tennessee Health Science Center. In May of 2012, she joined the laboratory of Dr. Taosheng Chen at St. Jude Children's Research Hospital to study the function and regulation of the nuclear receptor NR4A1 by miRNAs in cancer. She graduated in August of 2016 with the degree of Doctor of Philosophy with a concentration in cancer and developmental biology. She will pursue a postdoctoral fellowship in Tucson, AZ. 